Research Report

\title{
Considering Heroin-Assisted Treatment and Supervised Drug Consumption Sites in the United States
}

Beau Kilmer, Jirka Taylor, Jonathan P. Caulkins, Pam A. Mueller, Allison J. Ober, Bryce Pardo, Rosanna Smart, Lucy Strang, Peter H. Reuter 
For more information on this publication, visit www.rand.org/t/RR2693

Published by the RAND Corporation, Santa Monica, Calif.

(C) Copyright 2018 RAND Corporation

RAND $^{\circledR}$ is a registered trademark.

\section{Limited Print and Electronic Distribution Rights}

This document and trademark(s) contained herein are protected by law. This representation of RAND intellectual property is provided for noncommercial use only. Unauthorized posting of this publication online is prohibited. Permission is given to duplicate this document for personal use only, as long as it is unaltered and complete. Permission is required from RAND to reproduce, or reuse in another form, any of its research documents for commercial use. For information on reprint and linking permissions, please visit www.rand.org/pubs/permissions.

The RAND Corporation is a research organization that develops solutions to public policy challenges to help make communities throughout the world safer and more secure, healthier and more prosperous. RAND is nonprofit, nonpartisan, and committed to the public interest.

RAND's publications do not necessarily reflect the opinions of its research clients and sponsors.

Support RAND

Make a tax-deductible charitable contribution at www.rand.org/giving/contribute

www.rand.org 
Current levels of opioid-related morbidity and mortality in the United States are staggering. Data for 2017 indicate that there were more than 47,000 opioid-involved overdose deaths (roughly similar to deaths from AIDS at its peak in 1995), and one in eight adults now reports having had a family member or close friend die from opioids. There has been a near-universal call from blue-ribbon commissions and expert panels for increasing access to Food and Drug Administration-approved medications for those with an opioid use disorder; however, jurisdictions addressing opioid use disorders and overdose may wish to consider additional interventions beyond increasing access to these medications. Two interventions that are implemented in some other countries but not in the United States are heroin-assisted treatment (HAT) and supervised consumption sites (SCSs). Given the severity of the opioid crisis, there is urgency to evaluate tools that might reduce its impact and save lives.

This mixed-methods report assesses evidence on and arguments made about HAT and SCSs and examines some of the issues associated with implementing them in the United States. The target audiences include decisionmakers in rural and urban areas grappling with overdoses and poisonings, as well as researchers and journalists. This report consolidates and builds on insights from four working papers about HAT and SCSs, including (1) a review of the HAT literature, (2) a review of the SCS literature, (3) a paper on international experience with the implementation of HAT and SCSs, and (4) a paper on key informant views on the acceptability and feasibility of implementing HAT and SCSs in selected U.S. jurisdictions heavily affected by the opioid crisis.

\section{RAND Ventures}

The RAND Corporation is a research organization that develops solutions to public policy challenges to help make communities throughout the world safer and more secure, healthier and more prosperous. RAND is nonprofit, nonpartisan, and committed to the public interest.

RAND Ventures is a vehicle for investing in policy solutions. Philanthropic contributions support our ability to take the long view, tackle tough and often-controversial topics, and share our findings in innovative and compelling ways. RAND's research findings and recommendations are based on data and evidence, and therefore do not necessarily reflect the policy preferences or interests of its clients, donors, or supporters.

Funding for this venture was provided by gifts from RAND supporters and income from operations. 


\section{Contents}

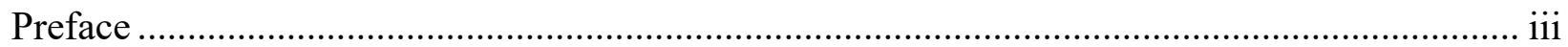

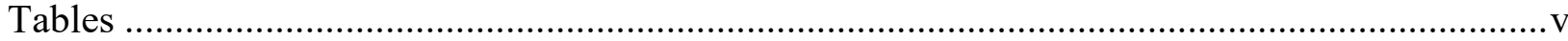

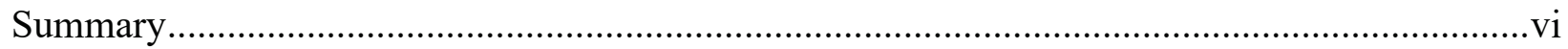

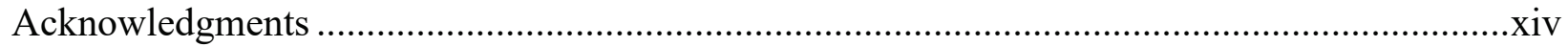

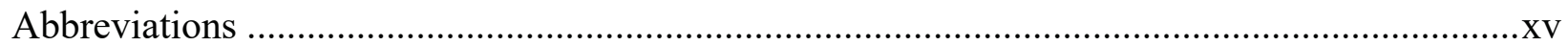

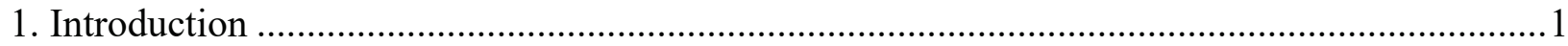

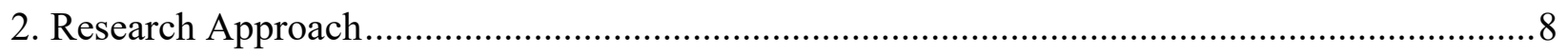

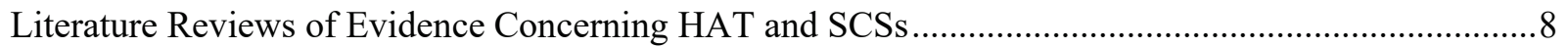

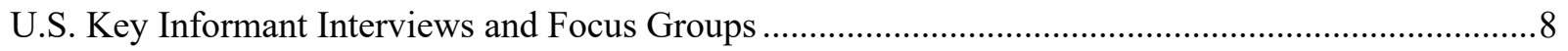

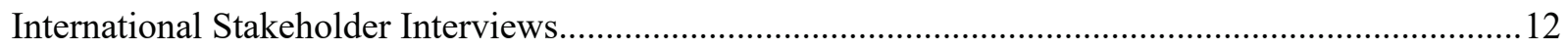

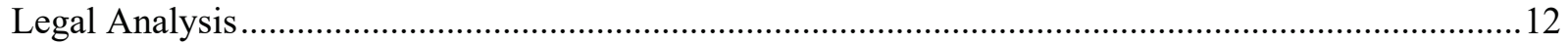

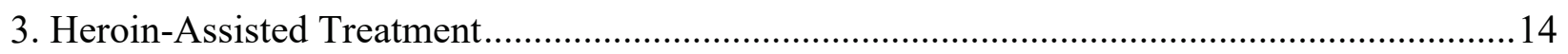

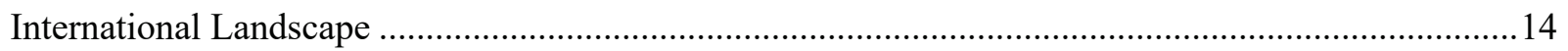

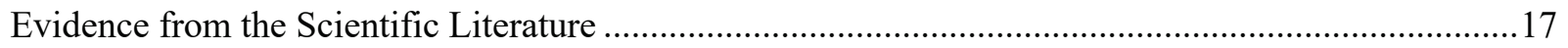

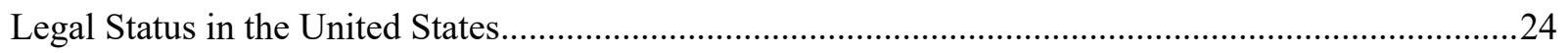

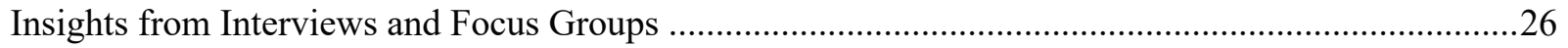

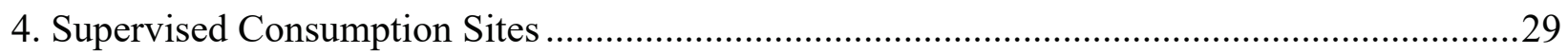

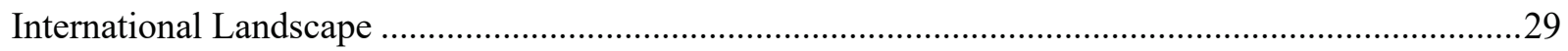

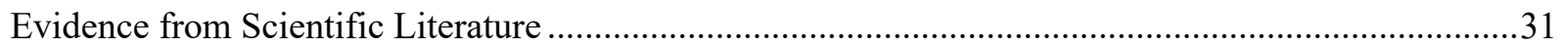

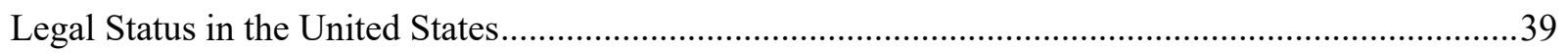

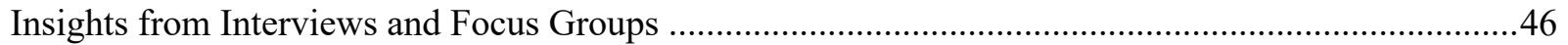

5. Ideas for Informing Future Research and Policy Discussions............................................49

Conducting Clinical Trials of HAT and Hydromorphone in the United States ...................................49

Implications of the Nature of Evidence Concerning the Effects of SCSs ............................................49

Potential Limitations Concerning the Scale of These Interventions ....................................................53

Are HAT and SCS Archetypes of Broader Collectives of Strategies?.................................................55

Appendix. A Brief History of Heroin-Assisted Treatment Discussions in the United States .......57

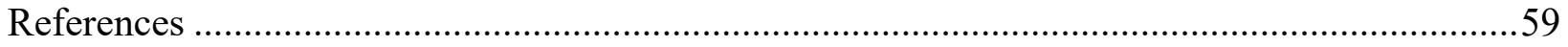




\section{Tables}

Table 2.1. Characteristics of Selected Counties .................................................................. 9

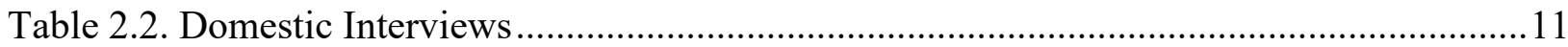

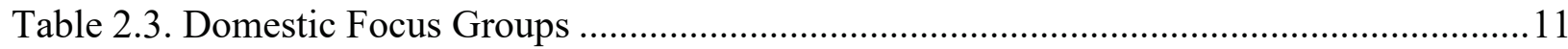

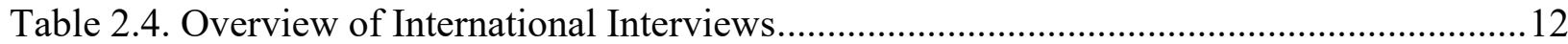

Table 3.1. International Provision of HAT ...................................................................... 14

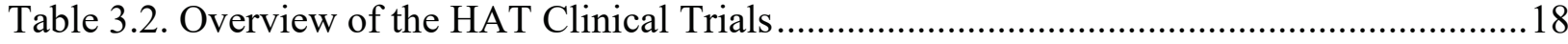

Table 3.3. Average Costs per Participant in Different Treatment Arms, Within-Trial

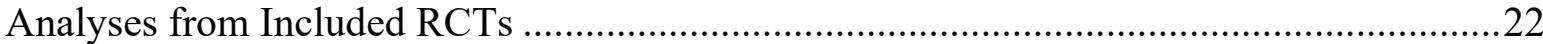

Table 3.4. HAT Themes Cited by Key Informants (Professionals and PWUO)........................27

Table 4.1. Supervised Consumption Sites Around the World....................................................30

Table 4.2. Quasi-Experimental Evaluations of Supervised Consumption Sites ........................32

Table 4.3. SCS Themes Cited by Key Informants (Professionals and PWUO) .........................47 


\section{Summary}

Current levels of opioid-related morbidity and mortality in the United States are staggering. Data for 2017 indicate that there were more than 47,000 opioid-involved overdose deaths (roughly similar to deaths from AIDS at its peak in 1995; Centers for Disease Control and Prevention, 2011, 2018a), and the actual figure is likely 20 to 35 percent higher because death certificates do not always list specific drugs (Ruhm, 2018). Furthermore, these fatalities exclude those who die from complications from infectious diseases, such as HIV or hepatitis C, contracted by sharing injection equipment and do not account for the costs associated with opioid use disorder (OUD) and nonfatal overdoses. In contrast to every other developed country, overall life expectancy is dropping in the United States, with opioids being an important driver (Dowell et al., 2017; Centers for Disease Control and Prevention, 2018b). Moreover, one in eight adults now reports having had a family member or close friend die from a prescription painkiller or heroin overdose (Associated Press-NORC Center for Public Affairs, 2018).

Since 2014, the increase in opioid-related deaths is primarily associated with illicitly produced synthetic opioids (e.g., fentanyl) being mixed into heroin and counterfeit pills (Centers for Disease Control and Prevention, 2017a). Because fentanyl and other synthetic opioids are much cheaper per morphine-equivalent dose and increasingly accessible from foreign suppliers, that pattern could well spread. The potential diffusion of these substances into nonopioid drug markets, such as for methamphetamine and cocaine, is also alarming.

Several blue-ribbon commissions and expert panels have made recommendations about how to mitigate the harms from opioids. There has been a near-universal call from these bodies for expanding access to medication treatments (MTs) for OUD approved by the Food and Drug Administration (FDA), such as methadone and buprenorphine (e.g., National Academies of Sciences, Engineering, Medicine, 2017; Christie et al., 2017). Although progress has been made on this front (Dick et al., 2015; Alderks, 2017), challenges remain, including a dearth of providers and lack of insurance coverage for some populations. Increasing the availability and reducing the costs for these medications are imperative.

Two additional interventions for addressing opioid-related harms that are implemented in other countries but not in the United States are heroin-assisted treatment (HAT) and supervised consumption sites (SCSs). Heroin has been prescribed in other countries for people whose OUD has not been effectively managed with other interventions, such as methadone. ${ }^{1}$ In these countries, the injection of pharmaceutical-grade heroin is typically supervised by medical officials in a designated health facility. ${ }^{2}$ In one sense, this is similar to the use of well-studied

\footnotetext{
${ }^{1}$ Heroin is also referred to as diamorphine or diacetylmorphine.

${ }^{2}$ This approach is often referred to as HAT, but other labels include supervised injectable heroin treatment, heroin maintenance, heroin prescription, or diamorphine prescription.
} 
and long-accepted medications such as methadone: Offer a legal, quality-controlled, free or lowcost pharmaceutical opioid as a replacement for expensive illicit market opioids of unknown potency whose acquisition may lead users to commit crime or potentially put themselves in dangerous situations. However, unlike methadone, which has a longer half-life and - if properly dosed - is unlikely to induce euphoria among those with tolerance to opioids, prescription heroin is typically dosed at levels that produce the desired effects among patients.

SCSs (sometimes referred to as drug consumption rooms, safe injection facilities, supervised consumption facilities, or overdose prevention sites) are places where individuals can consume already-purchased street drugs using sterile injection supplies in the presence of trained staff who monitor for overdose or risky injection practices, intervening when necessary. Some SCSs also provide additional services, such as referrals to treatment and access to drug content testing (which can identify dangerous adulterants, such as fentanyl, in powders sold as heroin). SCSs typically also supply take-home injection equipment for those injecting at the facility, as well as for those who do not.

HAT and SCSs have been implemented in Europe and Canada; Australia opened an SCS in 2001 and considered HAT but did not deploy it. However, there are barriers to implementing either in the United States. Heroin is listed as a Schedule I drug under the U.S. Controlled Substances Act (CSA), meaning that it has no currently accepted medical use and cannot be prescribed; however, it is possible to conduct human research with heroin and other Schedule I drugs. The legal status of SCSs is also complicated because the CSA forbids making any place available for unlawful distribution or use of a controlled substance (21 U.S.C. 856). Indeed, the U.S. attorney for Vermont has specifically denounced one county's intention to open an SCS (U.S. Department of Justice, U.S. Attorney's Office, District of Vermont, 2017), and in April 2018, a Drug Enforcement Administration spokesperson told the Washington Post that SCSs violate the CSA and are "subject to being prosecuted" (Cunningham, 2018). More recently, Deputy Attorney General Rod Rosenstein (2018) published an op-ed in the New York Times arguing that SCSs "create serious public safety risks," "normalize drug use and facilitate addiction," and do not help people who use opioids (PWUO) to stop using. That said, the American Medical Association (2017) has called for SCS pilot studies, and more than a dozen U.S. cities are considering allowing SCSs (Lopez, 2018).

This mixed-methods report assesses the evidence on and arguments made about HAT and SCSs and examines some of the issues associated with implementing them in the United States. This report consolidates and builds on insights from four working papers produced by RAND Corporation researchers as part of this project (Ober et al., 2018; Pardo, Caulkins, and Kilmer, 2018; Smart, 2018; Strang and Taylor, 2018) and a legal analysis. In addition to critically reviewing evidence reported in the scientific literature, we interviewed more than two dozen stakeholders in Canada, the Netherlands, Switzerland, and the United Kingdom to learn more about their experiences with these programs. Furthermore, to examine interest in and perceived barriers to implementing HAT and SCSs in New Hampshire and Ohio-two of the states hit hardest by the opioid crisis - we conducted interviews and focus groups with various types of 
key informants, including policy professionals, frontline service providers, and people who use heroin or other opioids.

\section{Key Insights}

- Although heroin cannot be prescribed in the United States because it is a Schedule I drug, it would be legal under federal law to conduct human research, including randomized controlled trials (RCTs), on HAT. Until the passage of the Harrison Act of 1914 and subsequent Supreme Court decisions, doctors could prescribe maintenance doses to heroin-dependent patients. The CSA, passed in 1970, placed heroin in Schedule I, meaning that it can be the object of human research studies but not prescribed for treatment of a qualifying condition. In theory, a state could legalize medical heroin, just as many states have legalized medical cannabis, although the probability of finding a pharmaceutical company (or other entity - e.g., a state university) to openly violate federal law and produce or import the heroin seems very slight.

- Evidence from RCTs of HAT in Canada and Europe indicates that HAT is better in some circumstances than methadone. Evidence from all studies reviewed indicates that supervised injectable HAT with optional oral methadone can offer benefits over oral methadone alone for treating OUD among individuals who have tried traditional treatment modalities, including methadone, multiple times but are still injecting heroin. The strongest and most consistent effects across studies are shown for reducing illicit heroin use and improving treatment retention. These are important findings given the current harms people who use heroin face from exposure to fentanyl and other synthetic opioid in many illicit markets. However, one reason HAT treatment retention rates were higher than methadone treatment retention rates is that some participants randomly assigned to oral methadone immediately dropped out of treatment. Another is an asymmetry caused by defining retention as remaining in any treatment; HAT participants can (and do) switch to methadone, but the converse was not permitted in the trials. Thus, HAT in some sense had a built-in advantage on retention measures.

There is also suggestive evidence that, within these populations, HAT may be more effective than oral methadone in reducing individuals' criminal activity and illicit use of benzodiazepines, as well as for improving their physical and mental health. ${ }^{3}$ But there is strong evidence indicating that HAT carries significantly greater risk than oral methadone for serious adverse events. Clearly, any relative benefits of HAT must be balanced with the risks associated with it. Still, the risk of such adverse events is likely to be much smaller for HAT than with the use of street-sourced heroin or synthetic opioids, whose potency and consistency are virtually unknown to consumers. Since the target audience is those who had already tried traditional treatments, the more relevant comparison may be HAT versus street-sourced opioids.

- Although HAT is more expensive than oral methadone, HAT may be more costbeneficial for clients who are refractory to standard treatments, largely through

\footnotetext{
${ }^{3}$ In our review of the comparative effectiveness of HAT for patient-level outcomes, we consider the evidence base as showing strong support if all or almost all studies assessed comparative effectiveness for a given outcome, studies of comparable methodological quality did not find significant effects in opposing directions, and more than twothirds of the relevant studies found significant effects in the same direction. If this third factor did not hold but statistically insignificant findings generally supported the same direction of the effect, we consider the evidence as suggestive.
} 
HAT's effect on reducing criminal activity among its target population. In the United States, methadone is only supplied as an oral medication. Supervised injectable HAT is much more expensive to administer than oral methadone mainly because of the staffing requirements associated with the supervision of injections, but it may still offer a better societal benefit-cost ratio for those who have previously undergone treatment but were still injecting heroin. This is primarily because evaluations in the literature credit HAT with doing more to reduce participants' levels of criminal involvement. Most trials also showed that users in the HAT arm enjoyed higher quality-adjusted life years (QALYs) relative to those who were assigned to oral methadone. However, that distinction may come from the greater supervision or the mode of administration rather than the chemical; among the two studies comparing the cost-effectiveness of supervised injectable heroin to other supervised injectable medications (injectable methadone or injectable hydromorphone), there were no significant differences between treatments in terms of costs or QALYs.

- Under CSA provisions for researching Schedule I drugs, HAT trials should be conducted in some of the U.S. jurisdictions that already provide a spectrum of social services and good accessibility to MTs for OUD. Given (1) the increased mortality associated with fentanyl, (2) the fact that some people who use heroin may not respond well to existing medications for OUD, (3) HAT's successful implementation abroad, and (4) questions concerning whether the success would carry over to the United States, it would be informative to conduct a series of HAT RCTs in some of the places that provide a spectrum of social services and good accessibility to MTs for OUD. In these studies, the injection of pharmaceutical-grade heroin would be supervised by medical officials in a designated health facility. Conducting a multisite research study might also allow HAT to proceed in a limited fashion without requiring its schedule status to be changed and would provide evidence to inform such a rescheduling decision.

- The literature on the effectiveness of treating OUD with hydromorphone (e.g., Dilaudid) is less extensive than the literature on heroin, but the early results are encouraging. Studies of hydromorphone would face fewer barriers in the United States than HAT studies would because hydromorphone is a Schedule II drug. For example, the results of the first six-month phase of one Canadian RCT $(N=202$ participants) suggest that supervised injectable heroin and supervised injectable hydromorphone showed similar effectiveness with regard to treatment retention, illicit heroin use, illegal activities, physical health, and mental health. Additionally, the hydromorphone group had significantly fewer serious adverse events related to the study medication, compared with the HAT group.

Some U.S. jurisdictions and researchers may find injectable hydromorphone more attractive than HAT, given its regulatory status (and likely greater community acceptability than heroin), while others may prefer to evaluate HAT, given its much larger evidence base. Conducting trials with HAT and hydromorphone are not mutually exclusive options, and it may make sense to include them in the same study as was done in Canada. Assessing the impact of injectable hydromorphone via clinical trials (with or without a HAT arm) would inform future regulatory decisions about using it as a medication treatment for OUD.

- There are significant legal issues surrounding the implementation of SCSs in the United States. The federal CSA prohibits possession of heroin and many other controlled substances, and the CSA also forbids making any place available, "with or without compensation, ... for the purpose of unlawfully manufacturing, storing, distributing, or 
using a controlled substance" (21 U.S.C. 856). States seeking to implement SCSs could make counterarguments (e.g., that states have authority to operate SCSs under their police powers because protecting public health is a power reserved to the states), but this would not necessarily prevent the federal government from initiating criminal or civil enforcement actions against the operators of an SCS. There are also important legal questions about liability (e.g., whether the SCS would face liability if a client left a site under the influence and caused harm) and whether professional medical staff would be at risk of losing their licenses or prescribing privileges.

- Overall, the scientific evidence about the effectiveness of SCSs is limited in quality and the number of locations evaluated. There are no published RCTs evaluating an SCS's impact on individual and population-level outcomes. That is understandable because SCSs are often opened in response to crises, not as part of a research program. It is nonetheless problematic because some estimates of the cost-effectiveness of SCSs presume spillover effects on behavior outside the SCSs, and the extent of those indirect effects is hard to know without a controlled trial. Our review identified nine studies that evaluate the opening of SCSs as natural experiments with comparison cases, but these nine were based on just four SCSs in three cities: Sydney, Australia (five studies); Vancouver, Canada (two studies); and Barcelona, Spain (two studies). Furthermore, four of the nine are arguably superseded by later studies of the same events but using better methods or longer time series, in some sense reducing the effective number of evaluations to five. Also, the events were not ideal natural experiments because other relevant changes occurred around the same time. Most reviews of the SCS literature include studies that employ research methodologies that inadequately control for unobserved factors that may bias results. For example, the reviews often include studies that lack comparison groups or only compare frequent SCS users with those who use SCSs less frequently, making the studies vulnerable to selection bias. Such studies can provide useful background information about client characteristics and some other factors, but they are not well suited for drawing causal inferences regarding the net effects of SCSs. (These studies are discussed in the accompanying working paper by Pardo et al., 2018.)

Many SCSs have been around for 15 to 30 years and have survived multiple changes in local and national governments. Of course, persistence does not imply effectiveness; yet it seems unlikely that these programs - which were initially controversial in many places - would have such longevity if they had serious adverse consequences for their clients or for their communities. There are researchers and advocates who believe that during an emergency such as the present opioid crisis in the United States, the absence of a large downside risk for a program that has strong face validity may be sufficient for some decisionmakers to proceed, rather than waiting for further evidence. Nevertheless, if attempts to implement SCSs in the United States are successful, we hope that a strong research component is incorporated into these efforts.

- Estimating the overall effect of SCS on fatal and nonfatal overdoses is difficult. Over the past 30 years, thousands of overdoses occurring at SCSs have been reversed; the number in Vancouver's Insite alone exceeded 2,000 in 2017. These figures do not equate to the net population-level effect of operating an SCS because it is impossible to know what would have happened had the SCS never been opened; however, PWUO who overdose in the presence of trained staff equipped with naloxone are much more likely to have it reversed than if they overdosed on the same product without supervision. 
One method that has been used to address this question compares the trends in overdoses in neighborhoods with and without an SCS, before and after the SCS opened. Our review identified two studies with comparison groups examining fatal populationlevel overdoses: one based on Vancouver from 2001 to 2005 and another focused on New South Wales, Australia, from 1998 to 2006. The former reported a significant reduction in fatal drug overdoses in the area surrounding the SCS, and the latter did not; however, the latter did identify a significant decrease in opioid-involved emergency service calls in designated treatment areas relative to the rest of the state. Because the SCS in New South Wales opened during the Australian heroin drought, one must be careful about drawing strong conclusions about quasi-experimental studies conducted during this period.

The research on SCSs was mostly conducted in places and times when heroin was not often adulterated with potent synthetic opioids. Fentanyl and its various analogues increase the risk of overdose, which might make supervision more beneficial in places where it has penetrated the market. Another factor to consider is that, because fentanyl has a shorter duration of effect than traditional illicit opioids, individuals with OUD may be injecting more often (Peng and Sandler, 1999; Ciccarone, Ondocsin, and Mars, 2017), thus increasing the number of times each day they put themselves at risk of overdose.

- For drug consumption that is supervised, SCSs reduce the risk of disease transmission and other harms associated with unhygienic drug use practices; however, there is uncertainty about the size of the overall effect. Cross-sectional studies show that those who visit SCSs frequently also adopt safer injection practices outside the facility than those who come to SCSs less frequently. Some studies presume that this difference is caused by SCSs and find that these outside-the-building effects account for most of the SCSs' effect on disease transmission; however, the correlation could merely reflect heterogeneity across individuals, with more risk-averse users both frequenting SCSs and adopting safer practices outside SCSs. Furthermore, the mathematical simulation studies of SCSs' effects on disease transmission in a review by Kennedy, Karamouzian, and Kerr (2017) were almost all based on one location (Insite in Vancouver), which, like many SCSs, offers services beyond supervising consumption and providing sterile equipment for those consumption sessions. For example, Pinkerton (2010, 2011) credits Insite's syringe (or needle) exchange program (SEP) that provided sterile equipment for use outside Insite with preventing about 95 percent of the infections that were prevented by the SEP and SCS functions collectively.

- It is important to have a sense of potential scale limitations and costs when discussing HAT and SCSs. HAT is more expensive than conventional treatments, such as oral methadone, and its favorable evaluations pertain to clients who have previously entered conventional treatment but are still injecting heroin. This suggests that HAT's role may be to address individuals who are refractory to conventional treatment, not as a first-line treatment. Indeed, to date, only a small number of PWUO find HAT an attractive option, even in places where it is routinely offered (MacCoun and Reuter, 2011). Nonetheless, if one assumed that there were on the order of 1.5 million daily or near-daily heroin users in the United States and only 5 percent received HAT, that would equate to approximately 75,000 clients who may spend less in the illicit market, minimize their exposure to fentanyl, and possibly commit less crime or less frequently put themselves in dangerous situations to obtain drugs. Of course, there would be considerable financial, administrative, and likely political costs to get a HAT program of that size up and running. 
If these 1.5 million daily or near-daily heroin users injected an average of roughly twice a day (see Bayoumi and Zaric, 2008), that would be about 1.1 billion use sessions per year. Vancouver's Insite SCS supervised an average of 415 injections per day in 2017 (Vancouver Coastal Health, 2018), or roughly 150,000 per year. Even ignoring injection of methamphetamine and other drugs or the increased frequency of injecting associated with shorter-acting drugs such as fentanyl, this suggests that the United States would need more than 7,000 SCSs the size of Insite to supervise those use sessions, and, as of 2017, there were only about 100 SCSs operating worldwide. Of course, these figures tell us nothing about whether a local community should or should not adopt an SCS; they do, though, give a sense of scale and suggest that SCSs as implemented elsewhere would need to be part of a package of responses to the opioid crisis, not the only or primary piece.

Furthermore, some SCS models require significant start-up and operating costs. Although precise comparisons have not been conducted, the cost of supervising the consumption of someone who injects twice a day at the most widely studied SCS (Vancouver's Insite) might be in the same ballpark as the cost of providing methadone in the United States for the same duration. Of course, the costs of SCSs (and treatment) vary depending on the particular services offered, and the less structured overdose prevention sites that are becoming more popular in Canada are believed to cost much less per supervised injection.

- Despite noting potential benefits of both HAT and SCSs, key informants in four counties in Ohio and New Hampshire believed that the programs would face substantial resistance from community members, particularly in rural areas that lack basic treatment services and SEPs. In key informant interviews and focus groups, local attitudinal and political barriers were often mentioned as obstacles to HAT or SCS implementation. With respect to SCSs, interviewees mentioned concerns that individuals would be wary of using the service for fear of legal repercussions, especially in morerural areas where there is less anonymity. This observation echoes comments made by international interviewees who stressed that cooperation with law enforcement was critical for the implementation of SCSs. Furthermore, one recurring concern that was that both HAT and SCSs would perpetuate drug use and not address underlying addiction. Participants also raised concerns that PWUO are unlikely to travel far to an SCS to consume recently purchased drugs, limiting the usefulness of SCSs outside densely populated areas. Further, some key informants felt that community skepticism vis-à-vis HAT may mirror or amplify existing reservations about methadone and buprenorphine.

Despite some reservations and worries about HAT and SCS perpetuating use and concern about community acceptance, several key informants from all groups in New Hampshire and Ohio also felt that both HAT and SCSs could improve some outcomes for PWUO. Key informants in the more urban areas also noted that, despite initial resistance, these programs, like MTs, ultimately would be accepted if there is strong evidence. Many informants were interested in learning more about the evidence for these programs.

Although more than a dozen U.S. cities are contemplating SCSs (Lopez, 2018; Allyn, 2018), most are large metropolitan areas in coastal states with a political and public health context that is different from that of New Hampshire and Ohio. It is plausible that if we had focused our qualitative research on these cities, we would have heard different views. The selection of our focus states was driven by a desire to gather insights of stakeholders from areas that have been hit hard by the crisis (they ranked among the top three states in the country by age-adjusted opioid overdose death rates in 2016) and that 
have thus far not been covered in existing literature, which has predominantly focused on large urban centers.

- Although HAT and SCSs can be expected to be met with opposition from some stakeholder groups, international testimonies suggest that resistance tends to dissipate over time. Interviews with international key informants noted that there was often vocal opposition to SCSs when initially discussed or opened. This opposition revolved primarily around concerns about enabling drug use and potential negative community effects. However, according to interviewees, objections to SCSs from local stakeholders tended to disappear following their implementation, something that was also observed in numerous places that opened SEPs (Tempalski, 2007). In Switzerland, the Netherlands, and British Columbia, both HAT and SCSs are generally accepted services for people with OUD. In the United Kingdom, discontinuation of HAT trials was related to cost rather than stakeholder opposition.

- It might be constructive to view HAT and SCSs as exemplars of a broader strategy, not as the only option within their class. Part of the innovation of HAT might have been offering heroin, but part might have been that it was injected, that consumption was supervised, or that clients then had a choice of medications (heroin or methadone). Indeed, some clinical trials suggest that supervised injectable hydromorphone may be as effective as HAT, and hydromorphone has the advantage of being a Schedule II drug, not Schedule I, like heroin is.

SCSs currently supervise a very small proportion of all injection sessions even in cities such as Vancouver, where they are well established. Canadian cities have recently expanded the scale of SCSs by establishing a larger number of smaller overdose prevention sites that typically offer fewer services than formal SCSs but focus on the core provision of supervision of injections, naloxone and oxygen administration, and calls to emergency services. It may even be worth asking whether the benefits of supervised consumption sites depend on there being a physical brick-and-mortar site, which may be a lightning rod for opposition, or if the key is that consumption is supervised and whether there are other ways to get more opioid consumption supervised. 


\section{Acknowledgments}

We are deeply indebted to more than 180 people who shared their experiences and opinions with us during interviews and focus groups. We are thankful to those in New Hampshire and Ohio who helped us set up the focus groups and to Martin Iguchi for making introductions. We are also very grateful for the detailed feedback we received from Leo Beletsky, Ricky N.

Bluthenthal, Susan S. Everingham, Keith Humphreys, Paul Koegel, Melinda Moore, Nicholas M. Pace, and Brendan Saloner. We also thank Howard Shatz for the tremendous support and advice provided throughout the project. The views presented here are those of the authors only. 


\section{Abbreviations}

AIDS

CDC

CSA

DEA

DND

FDA

HAT

HIV

iOAT

MMT

MSIC

MT

NAOMI

NIMBY

ODWDA

OUD

PWUO

QALY

RCT

RIOTT

SALOME

SCS

SEP acquired immunodeficiency syndrome

Centers for Disease Control and Prevention

Controlled Substances Act

Drug Enforcement Administration

daily or near-daily

U.S. Food and Drug Administration

heroin-assisted treatment

human immunodeficiency virus

injectable opioid agonist treatment

methadone maintenance treatment

Medically Supervised Injecting Centre

medication treatment

North American Opiate Medication Initiative

not in my backyard

Oregon Death with Dignity Act

opioid use disorder

people who use opioids

quality-adjusted life year

randomized controlled trial

randomized injecting opioid treatment trial

Study to Assess Longer-term Opioid Medication Effectiveness

supervised consumption site

syringe (or needle) exchange program 


\section{Introduction}

Current levels of opioid-related morbidity and mortality in the United States are staggering. Data for 2017 indicate there were more than 47,000 opioid-involved overdose deaths (roughly similar to deaths from AIDS at its peak in 1995; Centers for Disease Control and Prevention [CDC], 2011, 2018a), and the actual figure is likely 20 to 35 percent higher because death certificates do not always list specific drugs (Ruhm, 2018). Further, these fatalities exclude those who die from complications from infectious diseases, such as HIV or hepatitis C, contracted by sharing of injection equipment and do not account for the costs associated with opioid use disorder (OUD) or nonfatal overdoses. In contrast to every other contemporary developed country, overall life expectancy is dropping in the United States, with opioids being an important driver (Dowell et al., 2017; CDC, 2018b). ${ }^{1}$ Moreover, one in eight adults now reports having had a family member or close friend die from opioids (Associated Press-NORC Center for Public Affairs, 2018).

Since 2014, the increase in opioid-related deaths is attributable mainly to illicitly produced synthetic opioids (e.g., fentanyl) being mixed into heroin and counterfeit pills (CDC, 2017a), and there are reports that fentanyl has replaced heroin in some areas (see, for example, Mars, Ondocsin, and Ciccarone, 2018). ${ }^{2}$ Because fentanyl is much cheaper per morphine-equivalent dose and increasingly available from foreign suppliers, that pattern could well spread. But it would be incorrect to attribute these deaths only to fentanyl; many of these individuals were initially dependent on prescription opioids and only later traded down to street drugs, including counterfeit pills or fentanyl-laced heroin (Mars et al., 2014; Pollini et al., 2011). There are also the underlying social and economic conditions that contributed to some individuals becoming addicted to opioids in the first place (e.g., Case and Deaton, 2015; Kolodny et al., 2015; Dasgupta, Beletsky, and Ciccarone, 2018).

The United States will likely deal with the consequences of the large increase in OUD for many years. The nation's data infrastructure for tracking opioid users is not strong and has deteriorated in recent years (Caulkins et al., 2015), but building on previous estimates of daily and near-daily heroin users (Kilmer et al., 2014) and combining with estimates from a general population survey (Substance Abuse and Mental Health Services Administration, 2017) suggests that the number of individuals with OUD was well over 2 million in $2016 .{ }^{3}$ Even if the number

\footnotetext{
${ }^{1}$ As this report was being published, it was reported that opioid overdoses in British Columbia have led to an overall reduction in life expectancy in the province (Weeks, 2018).

${ }^{2}$ There is additional concern that people who use drugs other than opioids may be at risk of coming into contact with drugs laced with potent synthetic opioids (Drug Enforcement Administration [DEA], 2017; Jones, Einstein, and Compton, 2018).

${ }^{3}$ Based on the National Survey on Drugs and Health, the Substance Abuse and Mental Health Services Administration (2017) estimated that there are 2.1 million people age 12 or older who had an OUD in 2016;
} 
of users who escalate to OUD were suddenly reduced, available research on heroin-using careers suggests the current population of heavy users will impose serious costs to themselves and others for decades to come (Hser et al., 2001). Yet the introduction of potent synthetic opioids to the market is likely to change those drug-use careers and costs in ways that are still not fully understood.

A number of blue-ribbon commissions and expert panels have made recommendations about how to mitigate the harms from opioids. There has been a near universal call from these bodies to increase access to medication treatments (MTs) approved by the U.S. Food and Drug Administration (FDA) for OUD, such as methadone and buprenorphine (e.g., National Academies of Sciences, Engineering, and Medicine, 2017; Christie et al., 2017). Although progress has been made on this front (e.g., Dick et al., 2015; Alderks, 2017), challenges remain, including a dearth of providers and complicated reimbursement mechanisms. (See Box 1.1 for more information about these medications.) Increasing the availability and reducing the costs for these medications must remain a top priority.

There has also been a lot of discussion about supply-side interventions intended to prevent OUD and overdose, such as reducing high-risk opioid prescribing (e.g., morphine-equivalent daily dose of $100 \mathrm{mg}$ or more for more than six days, prescribing multiple opioids, opioidbenzodiazepine co-prescribing; Heins et al., 2018), shutting down "pill mills," and strengthening prescription drug monitoring programs. Although supply control can play an important role (see Pacula and Powell, 2018a, and the follow-up discussions by Saloner and Barry, 2018; Pacula and Powell, 2018b), policymakers must be cognizant of the possibility that reducing supply without a simultaneous increase in OUD treatment access could lead some people who use prescription opioids to seek out street-sourced opioids, which are often more dangerous.

Some U.S. jurisdictions have also pursued policies focused more on reducing the harm from opioid consumption than on the consumption itself. The traditional and long-standing example is syringe (or needle) exchange programs (SEPs), where people who inject drugs can exchange used syringes for sterile ones. ${ }^{4}$ Such programs are intended to reduce the spread of HIV and hepatitis and minimize other health harms associated with unhygienic injection (e.g., endocarditis) and can also serve as a useful contact point for users seeking treatment or other services. ${ }^{5}$ SEPs are not allowed in some parts of the United States, with the CDC (2017a) reporting that, as of 2016, roughly half of all states still did not authorize them.

\footnotetext{
however, the survey is notorious for missing those who use heroin on a daily or near-daily (DND) basis. Caulkins et al. (2015) showed that, while the National Survey on Drugs and Health estimated that there were about 60,000 DND heroin users in 2010, the actual figure was closer to 1 million.

${ }^{4}$ In some SEPs, an exchange does not have to take place; people who use opioids (PWUO) can simply pick up sterile equipment for free.

${ }^{5}$ There have been so many studies of SEPs that there are now systematic reviews of SEP systematic reviews and meta-analyses (e.g., see Palmateer et al., 2010; Fernandes et al., 2017).
} 


\section{Box 1.1. FDA-Approved Medications Currently Offered for Treatment of OUD}

Methadone: Methadone is a full mu-opioid receptor agonist with a long duration of action. If dosed properly, its pharmacological effects allow patients to avoid withdrawal symptoms that impair their daily functioning while blocking the effects of opioid agonists like heroin (National Institute on Drug Abuse, 2018a). Since 1972, this Schedule II substance has been permitted for managing OUD in opioid treatment programs. Methadone is available in tablets and syrups for daily use.

Over the past four decades, there have been many randomized controlled trials (RCTs), as well as several systematic reviews and meta-analyses, examining the effectiveness of methadone treatment. The most recent Cochrane systematic review examined $11 \mathrm{RCTs}$ and concluded that methadone was significantly more effective than nonpharmacological therapies, such as cognitive behavioral therapy, at retaining patients in treatment and reducing their use of heroin (Mattick et al., 2009).

Buprenorphine: Buprenorphine is a partial mu-opioid receptor agonist, meaning that it has partial efficacy at binding to the receptor, compared with a full agonist. Such partial agonist properties limit buprenorphine's euphoric and respiratory depressant effects. If dosed properly, buprenorphine allows patients to avoid withdrawal symptoms that impair their daily functioning while blocking the effects of opioid agonists like heroin (Center for Substance Abuse Treatment, 2004). Since the early 2000s, it has been permitted for managing OUD by prescribers in an office-based setting. Practitioners must receive certification (qualifying after an eight-hour course) before writing prescriptions to treat OUD and are capped at treating 275 patients. Buprenorphine can come in many formulations, sometimes with naloxone (e.g., Suboxone), which is intended to deter inappropriate use and prevent overdose. Formulations to treat OUD include tablets, sublingual or buccal films, and extended-release injections. Frequency of dosing is typically once a day, except for extended-release injections.

Since the 1990s, there have been dozens of RCTs and several systematic reviews that examine the effectiveness of buprenorphine versus other pharmacological treatments, including methadone. The most recent Cochrane systematic review examined 27 RCTs and concluded that buprenorphine is about as effective as methadone in ameliorating withdrawal and suggested no difference in average treatment duration (Gowing et al., 2017). Compared with other pharmacological treatments, such as clonidine and lofexidine, buprenorphine was found to be superior in treatment retention and reducing withdrawal severity.

Naltrexone: Naltrexone is a long-acting opioid receptor antagonist. Unlike methadone and buprenorphine, naltrexone binds to opioid receptors without stimulating them, blocking the effects of other opioids, including endorphins. Rather than substituting for illicit opioids, naltrexone is used to prevent detoxified patients from relapsing. Administering naltrexone to opioid-dependent individuals can precipitate withdrawal. In 1984, the FDA approved oral formulations for treating OUD. However, naltrexone's antagonist effects, which dampen or block the effects of endorphins, complicate treatment adherence (Fernandez and Libby, 2010), making daily oral administration infeasible for many patients. In 2010, the FDA approved an extended-release intramuscular injectable suspension formulation (e.g., Vivitrol) for treatment of OUD. These injections are often administered once a month. The cost of providing naltrexone medication can range, but treatment is generally more expensive than methadone and buprenorphine.

There are fewer studies evaluating extended-release formulations of naltrexone. The most recent Cochrane systematic review examined one RCT related to treatment of OUD with extended- 
release naltrexone versus placebo and nine nonexperimental studies. The authors reported that there was no significant difference in outcomes between those receiving extended-release injection naltrexone and those receiving the placebo. This finding and the remainder of other studies led authors to conclude that there is insufficient evidence of extended-release naltrexone for treating OUD (Lobmaier et al., 2008). A more recent RCT in Norway of 159 patients compared extended-release naltrexone with oral buprenorphine-naloxone and found no difference in treatment retention rates during the first 12 weeks (Tanum et al., 2017).

By contrast, there has been an infusion of funding to increase distribution of naloxone, a drug that can reverse opioid overdoses but does not reduce use, at least not directly. First responders in many states now routinely carry and administer naloxone, and, in some areas, authorities have permitted anyone to obtain and administer naloxone (Rowe et al., 2016). ${ }^{6}$ Data from Wheeler et al. (2015) suggest that, as of 2014, there were more than 600 community-based opioid overdose education and naloxone distribution programs operating in 30 states, and this number has likely increased given the current crisis (Rowe et al., 2018).

Nevertheless, some cities that have widespread naloxone provision and drug treatment availability are still suffering very high rates of opioid overdose deaths; Vancouver, Canada, is a prime example. Thus, there is interest in additional interventions. Two that are currently not implemented in the United States are heroin-assisted treatment (HAT) (also referred to as supervised injectable heroin treatment, heroin maintenance, heroin prescription, or diamorphine prescription) and supervised consumption sites (SCSs) (also known as drug consumption rooms, safe injection facilities, supervised consumption facilities, or overdose prevention sites $\left.{ }^{7}\right){ }^{8}$

Heroin has been prescribed in other countries for people whose OUD has proved refractory to other interventions, ${ }^{9}$ and the practice was allowed in the United States until the early 20 th century. ${ }^{10}$ In one sense, this is similar to the use of well-studied and long-accepted medications

\footnotetext{
${ }^{6}$ For more information about lay naloxone reversals, see Wheeler et al., 2015; Rowe et al., 2018.

7 The language used to describe this intervention may influence public acceptance (Barry, Sherman, and McGinty, 2018). Collins et al. (2018) argue,

Shifting away from "supervised injection facilities," terminology that risks evoking moralistic interpretations of harm reduction, the term 'Overdose Prevention Sites' refocuses attention away from perceived individual cause and control of drug use (Fraser, 2017) to the need for rapid interventions to address a public health issue.

In this report, we use SCS when referring to the traditional implementation of these programs and refer to overdose prevention sites to describe the new interventions in British Columbia and Ontario, as that is the language used in Canada to describe them.

${ }^{8}$ While SCSs are not legally allowed in the United States, there are reports of unsanctioned sites. For example, Lupick (2018) described bathroom sites in Boston and the Bronx and how "drug users take a strength-in-numbers approach by supervising one another" in Philadelphia's Kensington neighborhood. Kral and Davidson (2017) also presented detailed information about PWUO attending an unsanctioned SCS operating in an undisclosed location in the United States.

${ }^{9}$ Heroin is also referred to as diamorphine or diacetylmorphine.

${ }^{10}$ In the early 1900s, 34 U.S. cities hosted clinics that legally prescribed opiates to use for the purposes of maintenance. However, the 1914 Harrison Narcotic Act, which imposed a number of restrictions around the
} 
such as methadone: Offer a legal, quality-controlled, free or low-cost pharmaceutical opioid as a replacement for expensive illicit market opioids of unknown potency and whose acquisition may lead users to commit crime or potentially put themselves in dangerous situations. However, unlike methadone, which has a longer half-life and - if properly dosed — is unlikely to induce euphoria among those with OUD, prescription heroin is typically dosed at levels intended to produce the desired effects among patients. The immediate goal for HAT is to reduce the use of illicit heroin for those who have not been able to stop using heroin with other treatments. This is intended to stabilize the lives of people who use heroin, decrease their participation in the illicit market, and reduce their risk of overdose, disease transmission, and other health harms associated with using adulterated heroin and unsterile injection equipment. In the long run, HAT aims to keep clients engaged with treatment and improve their physical and mental health.

SCSs are places where individuals can consume illegal drugs in the presence of trained staff who monitor for overdose or risky injection practices and intervene when necessary. The emphasis on the word consume is important. SCS clients must obtain the drugs elsewhere; sale or distribution on site is not permitted. Some SCSs also provide additional services, including referrals to treatment and access to drug content testing (which can identify dangerous adulterants, such as fentanyl in bags sold as heroin). They typically also provide take-home injection equipment for those injecting at the facility, as well as for those who do not. The main goals of SCSs are to reduce the risk of overdose, disease transmission, and other health harms associated with unhygienic injections, especially in hard-to-reach and high-risk drug-using populations. SCSs also intend to reduce public consumption and are hypothesized to increase the utilization of social and medical services.

HAT and SCSs have been implemented in Europe and Canada; Australia opened an SCS in 2001 and considered HAT but did not deploy it. However, there are barriers to implementing each in the United States. Heroin is a Schedule I drug under the U.S. Controlled Substances Act (CSA), passed in 1970, meaning that it has no currently accepted medical use and cannot be prescribed; however, it is possible to conduct human research with heroin and other Schedule I drugs. To our knowledge, no study has so far examined the acceptability and feasibility of HAT in the United States to help address the current opioid crisis.

The legal status of SCS is also problematic because the CSA forbids anyone from making any place available, "with or without compensation, . . . for the purpose of unlawfully manufacturing, storing, distributing, or using a controlled substance" (21 U.S.C. 856), but states may nonetheless have authority to operate SCSs under their police powers because protecting public health is a power reserved to the states (e.g., Beletsky et al., 2008). Despite the threat of federal intervention, more than a dozen U.S. cities are considering allowing SCSs (Lopez, 2018; Allyn, 2018). There is some research that examines the attitudes and perspectives of PWUO and

administration of these drugs, was interpreted to prohibit the prescription of opiates for this purpose, and within a few years, doctors who had prescribed opiates to maintain users on a steady dose were being prosecuted under this law (European Monitoring Centre for Drugs and Drug Addiction, 2012). For information about HAT policy debates in the United States since then, see the appendix. 
other stakeholders regarding a potential introduction of SCSs in certain jurisdictions within the United States. For instance, Kral et al. (2010) conducted a survey of users in San Francisco to examine their willingness to use such a facility. Also in San Francisco, Wenger, Arreola, and Kral (2011) explored community response to a potential introduction of an SCS in the city's Tenderloin district, based on interviews with various stakeholders. These studies mirror similar explorations of stakeholder attitudes undertaken in international jurisdictions. For instance, the introduction of SCSs in Toronto and Ottawa had been preceded by a large body of research on stakeholder views (Bayoumi et al., 2012; Watson et al., 2012; Watson et al., 2013; Strike et al., 2014; Strike et al., 2015; Watson et al., 2015; Kolla et al., 2017). Although existing literature on stakeholder views focuses predominantly on large urban centers, some international studies have also gathered insights from smaller jurisdictions (e.g., Fischer and Allard, 2007; Bardwell et al., 2017; Mitra et al., 2017).

This mixed-methods report assesses evidence on and arguments made about HAT and SCSs and examines some of the issues associated with implementing them in the United States. It focuses on three main questions:

- What does the high-quality scientific evidence suggest regarding the effectiveness of HAT and SCSs?

- What are the legal considerations surrounding potential introduction of HAT and SCSs in the United States?

- What are the policy, technical, and community issues surrounding potential introduction of HAT and SCSs in the United States, particularly in areas hit hardest by the opioid crisis?

This report consolidates and builds on insights from four working papers produced by RAND researchers as part of this project (Ober et al., 2018; Pardo, Caulkins, and Kilmer, 2018; Smart, 2018; Strang and Taylor, 2018). In addition to reviewing evidence reported in the scientific literature, we interviewed more than two dozen key informants in Canada, the Netherlands, Switzerland, and the United Kingdom to learn about their experiences with these programs. We also performed legal analyses to determine what is permitted under current laws in the United States, with a focus on liability issues. Furthermore, to learn more about how the international experience may or may not generalize to the United States and about some of the practical issues associated with implementing these programs, we conducted focus groups with people who use street-sourced opioids (ten groups, 79 people total) as well as with frontline service providers (five groups, 36 people total) in two of the three states with the highest drug overdose death rates in 2016: New Hampshire and Ohio. We also interviewed 44 key informants in these states, including policymakers and professionals from the harm reduction, treatment, and criminal justice systems. Although the convenience sampling and restricted geographic coverage limit the external generalizability of the U.S. qualitative work, this information offers rich insights for four counties in two of the states that need the most help dealing with the opioid crisis.

The remainder of this report is structured as follows. The next chapter briefly describes the methodology employed for each of the supporting papers. Chapters 3 and 4 focus on HAT and 
SCSs, respectively, and follow a similar structure: international experiences, review of the literature, assessment of the legal issues surrounding the implementation of these interventions in the United States, and insights from stakeholder interviews and focus groups. Chapter 5 offers additional thoughts intended to inform future research activities and policy discussions. The appendix briefly describes the history of HAT-related discussions in the United States. 


\section{Research Approach}

This chapter briefly describes the methodologies employed by each of the papers supporting this report. More detailed descriptions are included in the individual reports (Ober et al., 2018; Pardo, Caulkins, and Kilmer, 2018; Smart, 2018; Strang and Taylor, 2018).

\section{Literature Reviews of Evidence Concerning HAT and SCSs}

We searched five electronic databases for documents from 1990 until January 17, 2018: PubMed, Embase, Web of Science, Scopus, and WorldCat for books, reports, and "grey literature." ${ }^{14}$ No language restrictions were imposed. We also screened reference lists of systematic reviews and narrative reviews identified during the study selection process and conducted forward and backward searches on key articles. Finally, experts in the field who were participating in interviews for the project were given the opportunity to pass along any sources they believed to be of relevance. Additional details about the search strategies and inclusion criteria are reported in Pardo, Caulkins, and Kilmer (2018) and Smart (2018).

\section{U.S. Key Informant Interviews and Focus Groups}

\section{Methodology for Choosing Jurisdictions}

We focused on one rural and one urban county within each of two states with some of the highest rates of opioid overdose deaths. ${ }^{15}$ Our main goal was to target both urban and nonurban jurisdictions that have been hit hard by the opioid crisis, as well as to select counties that would allow for regional and racial diversity. ${ }^{16}$ At the time of selection, the most-recent mortality data available for state-level comparisons were the 2016 age-adjusted opioid overdose death rates

\footnotetext{
${ }^{14}$ Grey literature refers to sources not published by a commercial publisher, such as government reports and civil society publications. For a more detailed definition, see Grey Literature Report, New York Academy of Medicine, undated.

${ }^{15}$ Because of budgetary constraints, we had to limit our inquiry to four counties in two states but still aimed to ensure diversity in our site selection.

${ }^{16}$ We felt that it was important to ensure some degree of racial diversity in the selected sample to capture stakeholder perspectives that may reflect various racial disparities pertaining to the opioid crisis and aspects such as drug arrests or possible differences in users' attitudes toward services for opioid users (see, for example, Howard, Barrett, and Holmes, 2010, for a brief overview of literature on possible attitudinal factors in race disparities in methadone treatment). Furthermore, although this was not an explicit selection criterion, the inclusion of various types of jurisdictions also increased the likelihood of capturing a multitude of local political climates, which play an important role in responding to public health issues. It is plausible that if we had focused our qualitative research on other jurisdictions (e.g., those whose leadership have publicly expressed interest in either intervention), we would have heard different opinions about the acceptability of implementing those programs from PWUO and other key informants.
} 
from the CDC. New Hampshire and Ohio ranked second and third, respectively (first was Ohio's neighbor West Virginia). ${ }^{17}$ In Ohio, using 2016 data from the CDC, Cuyahoga County (which includes Cleveland) had the largest number of opioid deaths, as well as the largest per capita rate among the state's large metropolitan counties (CDC, 2017b). Cuyahoga county's large African American population ensured some racial diversity in our analysis. Among nonmetropolitan counties (i.e., those defined by the National Center for Health Statistics urban-rural county classification scheme as micropolitan and noncore), Ashtabula County was tied for the largest absolute number of opioid deaths and fifth for per capita rates.

In New Hampshire, analyses of CDC data found that Hillsborough County (which includes the city of Manchester) had the largest absolute and per capita figures for opioid overdose deaths in the state. Among nonmetropolitan counties, Carroll County had the highest rate, and the absolute number (14) was similar to other nonmetropolitan counties except Merrimack, which had 34 overdose deaths (CDC, 2017b). Because Merrimack includes the capital and has a population of approximately 150,000 , we chose Carroll, which has a much smaller population. The characteristics of all four selected counties are summarized in Table 2.1.

Table 2.1. Characteristics of Selected Counties

\begin{tabular}{|c|c|c|c|c|}
\hline & $\begin{array}{l}\text { Population } \\
\text { (2016) }\end{array}$ & $\begin{array}{l}\text { Opioid-Related } \\
\text { Deaths per 100,000 } \\
\text { People (2016) }\end{array}$ & $\begin{array}{l}\text { Ranking in Counties in } \\
\text { Overdose Death Rate } \\
\text { in the State }\end{array}$ & $\begin{array}{c}\text { Percentage White } \\
\text { (2017 Estimate) }\end{array}$ \\
\hline Carroll County, NH & 47,289 & 29.6 & $\begin{array}{c}\# 4 \text { out of } 10 \text { (\#1 among } \\
\text { nonmetro) }\end{array}$ & 97.1 \\
\hline Hillsborough County, NH & 407,761 & 42.9 & $\# 1$ out of 10 & 90.7 \\
\hline Ashtabula County, $\mathrm{OH}$ & 98,231 & 38.7 & $\begin{array}{c}\# 13 \text { out of } 88 \text { (\#4 among } \\
\text { nonmetro) }\end{array}$ & 93.2 \\
\hline Cuyahoga County, $\mathrm{OH}$ & $1,249,352$ & 40.0 & $\begin{array}{l}\# 10 \text { out of } 88 \text { (\#1 among } \\
\text { large central metro) }\end{array}$ & 63.8 \\
\hline
\end{tabular}

SOURCES: CDC, 2017b; U.S. Census Bureau, 2018.

\section{Key Informant Interviews}

We conducted telephone interviews with 44 state- and county-level key informants. We identified initial interviewees via informal discussions with subject-matter experts and internet searches for individuals involved in efforts to address the opioid crisis. We then asked everyone we interviewed to recommend additional key informants at the state or county levels who were familiar with the opioid crisis and to provide contact information. Stakeholders included representatives from state and local governments and the criminal justice system, as well as harm

\footnotetext{
${ }^{17}$ We selected New Hampshire and Ohio because of a desire to have some geographical variation in the selected jurisdictions. The reason for selecting Ohio and not West Virginia was twofold. First, the absolute number of total opioid deaths in Ohio is many times higher than in West Virginia. Second, the selection of Ohio offers the possibility to select a county that is more racially diverse than is the national average.
} 
reduction (including first responders), medical, social service, and substance use disorder professionals.

We developed interview and focus group protocols that were designed to capture the following information: (1) perspectives on the nature and extent of the opioid problem within the county; (2) current services for PWUO and gaps in services; (3) prior knowledge of and opinions about the effectiveness of HAT and SCSs as ways to help address the opioid crisis in the county; ${ }^{18}$ (4) opinions about whether HAT and SCS would be acceptable to community members; (5) which facilitators, if any, could increase the acceptability of HAT and SCSs; and (6) any additional recommendations for addressing the opioid crisis. We first asked broad, openended questions within each domain to capture a range of perspectives, then probed for moredetailed responses.

As a general rule, two members of the research team conducted each interview, with one leading the interview and the other primarily taking notes. The interviewer read a consent form aloud and requested verbal consent from the participant to participate in the interview and to be recorded. All interviews were recorded and professionally transcribed.

\section{Provider and PWUO Focus Groups}

We established contact with social service, harm reduction, recovery, and substance use disorder treatment stakeholders in each county (some of whom were recommended by prior interviewees) who facilitated the planning of four types of focus groups: (1) frontline service providers, (2) PWUO who were actively using and not in treatment, (3) PWUO currently in treatment at a facility that offered MT for OUD, and (4) PWUO currently in treatment at a facility that did not offer MT. Our community contacts helped establish dates and times for the groups and facilitate recruitment. The contacts sent email invitations to all potential provider focus group participants and distributed fliers to potential PWUO focus group participants. All participation was voluntary and anonymous, in that we did not collect participant names. Providers and PWUO each were asked to complete a brief, anonymous survey to capture background characteristics. All focus group participants were provided with a meal during the focus groups. PWUO also were given a \$25 gift card for participating in the group. As with the

18 The definition of HAT used by interviewers and focus group facilitators to describe the intervention to key informants who were not familiar with HAT was as follows:

HAT is intended for people who have failed treatment (e.g., methadone) multiple times. The premise is the same as for well-studied and long-accepted medication-assisted treatments such as methadone maintenance: Substitute a legal, quality-controlled, free or low-cost pharmaceutical opioid for expensive illicit market opioids whose potency and purity are not known to the seller or user.

The definition of SCS used by interviewers and focus group facilitators to describe the intervention to key informants who were not familiar with SCS was as follows:

SCSs are places where users can consume already-purchased street drugs in the presence of trained staff (sometimes volunteers, sometimes health professionals), who monitor for overdose or risky injection practices. Clients can sometimes test their drugs, acquire clean injection supplies, and engage with social services. 
key informant interviews, the groups were conducted by two members of the research team and were recorded and professionally transcribed.

Interviews and focus groups were conducted between January and March 2018. All procedures were approved by RAND's Institutional Review Board (RAND Human Subjects Protection Committee). The breakdown of these interviews and focus groups by stakeholder type and county is provided in Tables 2.2 and 2.3. The interview guides and additional information about the methodology are available in Ober et al. (2018).

Table 2.2. Domestic Interviews

\begin{tabular}{lcccccccc}
\hline & \multicolumn{3}{c}{ New Hampshire } & & \multicolumn{3}{c}{ Ohio } & \\
\cline { 2 - 4 } \cline { 7 - 8 } Interview Participant & State & Rural & Urban & & State & Rural & Urban & Total \\
\hline $\begin{array}{l}\text { Criminal justice } \\
\text { professional }\end{array}$ & 0 & 3 & 1 & & 1 & 1 & 3 & 9 \\
$\begin{array}{l}\text { Harm-reduction } \\
\text { professional }\end{array}$ & 0 & 1 & 3 & & 0 & 0 & 3 & 7 \\
Medical professional & 0 & 0 & 0 & & 0 & 0 & 4 & 4 \\
Policy professional & 2 & 2 & 1 & & 4 & 1 & 4 & 14 \\
Treatment professional & 0 & 2 & 3 & & 1 & 4 & 0 & 10 \\
Total & $\mathbf{2}$ & $\mathbf{8}$ & $\mathbf{8}$ & & $\mathbf{6}$ & $\mathbf{6}$ & $\mathbf{1 4}$ & $\mathbf{4 4}$ \\
\hline
\end{tabular}

Table 2.3. Domestic Focus Groups

\begin{tabular}{|c|c|c|c|c|c|}
\hline \multirow[b]{2}{*}{ Focus Group } & \multicolumn{2}{|c|}{$\begin{array}{l}\text { Groups in New } \\
\text { Hampshire (and Number } \\
\text { of Participants) }\end{array}$} & \multicolumn{2}{|c|}{$\begin{array}{l}\text { Groups in Ohio } \\
\text { (and Number of } \\
\text { Participants) }\end{array}$} & \multirow[b]{2}{*}{ Total } \\
\hline & $\begin{array}{c}\text { Rural } \\
1(7)\end{array}$ & $\begin{array}{c}\text { Urban } \\
1(9)\end{array}$ & $\begin{array}{c}\text { Rural } \\
1(9)\end{array}$ & $\frac{\text { Urban }}{2(11)}$ & \\
\hline PWUO, active & 0 & 0 & $1(5)$ & $2(6)$ & $3(11)$ \\
\hline PWUO, MT & 0 & 0 & $2(23)$ & $1(12)$ & $3(35)$ \\
\hline PWUO, non-MT & $2(14)$ & $2(19)$ & 0 & 0 & $4(33)$ \\
\hline Total & $3(21)$ & $3(28)$ & $4(37)$ & $5(29)$ & $15(115)$ \\
\hline
\end{tabular}

\section{Data Analysis}

We used standard qualitative methods (Jehn and Doucet, 1996, 1997; Ryan and Bernard, 2000, 2003; Miles and Huberman, 1994; Willms et al., 1990) to identify themes around our primary research questions (perceived effectiveness of HAT and SCSs, acceptability to community members, and implementation). Two research team members read all transcripts to identify themes (overarching categories describing phenomena under study) across all stakeholder interviews and focus groups. Next, researchers drafted a codebook listing key themes, inclusion and exclusion criteria, and typical examples. Data were coded into Dedoose (a qualitative data management program) to extract themes and statements from interviews. 
Intercoder reliability was measured throughout the process to ensure consistency across coding of themes. For additional information, see Ober et al. (2018).

\section{International Stakeholder Interviews}

This analysis draws on 29 interviews conducted in the spring 2018 with key informants in Canada, the Netherlands, Switzerland, and the United Kingdom. The objective of the interviews was to gather insights on HAT and SCS initiatives and lessons learned from jurisdictions that have implemented one or both interventions. Three types of key informants were invited to an interview with the research team: (1) policymakers involved in the decisionmaking surrounding these initiatives, (2) practitioners involved in the management or delivery of these programs, and (3) researchers conducting scientific work on these programs. The breakdown of these interviews by stakeholder type and country is provided in Table 2.4 .

Our key informants were primarily recruited from groups who were involved in or could comment on the operation of HAT and SCSs and therefore largely held a positive view of the interventions. It is plausible that representatives of other stakeholder groups might have more reservations about the two types of programs. ${ }^{19}$ In addition, it was beyond the scope of the study to interview HAT and SCS clients in the selected focus countries; their perspectives are therefore missing from this report.

Table 2.4. Overview of International Interviews

\begin{tabular}{lcccc}
\hline Country & Policymaker & Practitioner & Researcher & Total \\
\hline Canada & 2 & 3 & 3 & 8 \\
Netherlands & 0 & 3 & 3 & 6 \\
Switzerland & 0 & 2 & 2 & 4 \\
United Kingdom & 3 & 4 & 4 & 11 \\
Total & $\mathbf{5}$ & $\mathbf{1 2}$ & $\mathbf{1 2}$ & $\mathbf{2 9}$ \\
\hline
\end{tabular}

For the international interviews, we applied the same methods as for interviews with U.S.based stakeholders. Once again, all procedures were approved by the RAND Human Subjects Protection Committee.

\section{Legal Analysis}

We consulted scholarly articles, cases, regulations, statutes, and government policy statements to analyze the legal barriers to HAT and SCSs in the United States and to understand

\footnotetext{
${ }^{19}$ That said, we did ask respondents about their views on the limitations of these approaches and their potential negative consequences.
} 
the potential liabilities a HAT or an SCS provider might face. Most of the issues raised by HAT and SCSs have not yet been directly addressed in case law. The reasoning in the analyses also draws on the literature regarding SEP, medical cannabis, nuisance laws, and dram shop liability. ${ }^{20}$

${ }^{20}$ Dram shop liability means that a business that sells alcoholic drinks (and, in some states, a host who serves drinks) to an obviously intoxicated person is strictly liable to anyone who is injured by the intoxicated individual. This is currently the law in a majority of states, either via statute or case law, although specifics are inconsistent across states. 


\section{Heroin-Assisted Treatment}

\section{International Landscape}

HAT has been implemented in a number of European countries and in Canada. As discussed in Strang and Taylor (2018), the particulars of HAT's implementation differ across countries; however, all the programs share the underlying premise to offer a legal, quality-controlled, free or low-cost pharmaceutical opioid as a replacement for illicit market opioids of unknown quality. Table 3.1 provides an overview of international HAT provision; in most countries, there are at least ten times as many methadone or buprenorphine clients as HAT clients, underscoring that HAT is somewhat of a niche program even in places where it is not controversial. In the next subsections, we offer additional details on HAT services in the four focus countries included in this study (in chronological order of when they first initiated HAT). Further details about HAT provision in these countries can be found in Strang and Taylor (2018).

Table 3.1. International Provision of HAT

\begin{tabular}{lccc}
\hline Country or Province & Number of HAT Clinics & Number of HAT Clients & Number of MT Clients \\
\hline Canada (British Columbia) & 1 & $150(2018)$ & $19,000(2015)$ \\
Denmark & 5 & $450(2015)$ & $7,050(2015)$ \\
Germany & 9 & $630(2016)$ & $78,500(2016)$ \\
Luxembourg & 1 & Not available (pilot & $1,085(2016)$ \\
Netherlands & 17 & introduced in 2017) & $5,241(2015)$ \\
Switzerland & 22 & 1,608 slots (2018) & $18,000(2018)$ \\
United Kingdom & Unknown & Estimated to be in the & $138,000(2016)$ \\
& \multicolumn{4}{c}{ hundreds } \\
\hline
\end{tabular}

SOURCES: British Columbia Ministry of Health, 2017; European Monitoring Centre for Drugs and Drug Addiction, 2017b, 2018a, 2018b, 2018c; Federal Office of Public Health, 2018.

NOTES: The numbers in this table represent the best available indicators and may be approximations. In addition to the countries listed, Spain offers HAT to some users under compassionate use provisions.

\section{United Kingdom}

In the United Kingdom, heroin prescription for the purpose of treating addiction has always been legal. It was officially sanctioned in the Rolleston Report (1926), where drug addiction was defined as an illness and, thus, treatment for it was the responsibility of the patient's doctor. The Dangerous Drugs Act of 1967 imposed a new requirement on doctors to obtain a license from the Home Office before they were permitted to prescribe heroin for this purpose (UK Parliament, 1967). This led to the creation of National Health Service addiction clinics, which were typically attached to hospitals. The practice of HAT diminished in the 1980s and 1990s because of a 
number of factors, including licensing requirements and a reluctance on the part of doctors to prescribe heroin for this purpose, particularly given the increasing availability of alternative medications, such as methadone. Currently, the number of doctors licensed to prescribe heroin across England and Wales is estimated to be in the double digits, and the number of licensed doctors who actually prescribe heroin may be smaller (European Monitoring Centre for Drugs and Drug Addiction, 2012).

Heroin prescription delivered under this system has historically been governed by few specific or enforceable national policies, and treatment protocols have been known to vary widely by clinician (Metrebian et al., 2002). However, clinicians must comply with the relevant legal obligations in relation to the prescribing of controlled drugs, such as heroin, as well as the terms of the Home Office licensing arrangements (Clinical Guidelines on Drug Misuse and Dependence Update 2017 Independent Expert Working Group, 2017). Furthermore, the 2017 Clinical Guidelines on Drug Misuse and Dependence Update set out the relevant standards and quality of care for the appropriate treatment of people with a substance use disorder, including heroin prescription, when assessing a clinician's professional performance. The 2002 UK Drug Strategy suggested that heroin prescription should be available to all patients with a clinical need (although this term was not defined) (UK Home Office Drugs Strategy Directorate, 2002).

The randomized injecting opioid treatment trial (RIOTT), which introduced HAT with supervised consumption and psychosocial care to the United Kingdom, ran from 2005 to 2008 at three sites: Durham, Brighton, and London. The Department of Health continued to fund these sites until 2013. These sites closed when the funding expired and have not been replaced since, although heroin prescription under the old "British system" continues to be available (and covered by the National Health Service). Furthermore, there are plans to roll out new HAT services in at least two UK areas (Durham and Glasgow).

\section{Switzerland}

In 1991, the Swiss government adopted a "Four Pillars" drug policy, which added harm reduction to the existing pillars of law enforcement, prevention, and treatment. One of the initiatives introduced under this new policy was a national HAT research study (Uchtenhagen, 2017). The trial was organized as a prospective cohort study and took place from 1994 to 1996 (Fischer et al., 2007). Following the publication of the results of the study, which showed improvements for participants in a range of outcomes, including illicit drug use, criminal activity, and mental and physical health (Uchtenhagen et al., 1999), the federal government issued an executive order allowing the continuation of the treatment and formalized HAT as an additional therapy for PWUO in 2003 (Khan et al., 2014). HAT was also confirmed as an available treatment option in a referendum of the Swiss Narcotic Law in 2008. Currently, there are 22 HAT clinics in the country, two of which are in prisons. Some clinics are administered by state authorities and the rest by nongovernmental organizations that have applied for and received approval to run a clinic and need to adhere to federal guidelines (Wuethrich and Drogen, 2015). The total capacity of HAT clinics in the country is approximately 1,600 patients. 
HAT serves less than 10 percent of all users receiving opioid agonist treatment (e.g., methadone; Uchtenhagen, 2017).

\section{Netherlands}

In the Netherlands, the first HAT clinics were established in 1998 as part of research trials recommended by the country's Council of Health aimed at patients who were severely dependent and had been unsuccessful with other available treatment options. The results of these trials were presented to the Ministry of Health in 2002; the following year, a special advisory committee installed by the government recommended that 15 HAT clinics across 13 cities be established. The Netherlands Medicines Evaluation Board registered heroin as a medicine for the treatment of this patient group in 2006. By 2009, HAT had become an established treatment option for users who had not benefited from methadone or buprenorphine maintenance treatment (Blanken et al., 2010). There are currently 17 HAT clinics across 16 cities in the Netherlands, serving around 650 patients every day (European Monitoring Centre for Drugs and Drug Addiction, 2018b).

\section{Canada}

The origins of HAT in Canada date back to a sequence of clinical trials, which started in the mid-2000s (Boyd, Murray, and MacPherson, 2017). A trial (called the North American Opiate Medication Initiative [NAOMI]) was established in the Crosstown clinic in Vancouver and in Montreal, running from 2005 to $2008 .{ }^{21}$ From 2011 to 2014, the NAOMI trial at Crosstown was followed by the SALOME (Study to Assess Longer-term Opioid Medication Effectiveness) trial, which compared the effectiveness of providing injectable diamorphine (heroin) and injectable hydromorphone (better known by its trade name, Dilaudid). The Conservative government attempted to block the continuation of heroin treatment after the conclusion of the SALOME trial, but the British Columbia Supreme Court issued an injunction, allowing the clinic to continue with the program out of trial (Providence Health Care Society v. Canada). In August 2017, Crosstown expanded the provision of injectable heroin to any clinically indicated patients - that is, extending the provision beyond the population of the SALOME trial. This step followed an earlier relaxation of rules by Health Canada on the importation of heroin for patients with exceptional needs. ${ }^{22}$

Currently, Crosstown continues to be the only clinic offering diamorphine treatment in North America. In addition to Crosstown, several other injectable opioid agonist treatment (iOAT) programs embedded in existing facilities opened recently in British Columbia. They provide, among other services, injectable hydromorphone and slow-release morphine. Another model of

\footnotetext{
21 The introduction of HAT precedes the approval of a buprenorphine and naloxone combination for OUD treatment, which was done by Health Canada in 2007 (Ahamad et al., 2016).

22 Currently, Crosstown imports diamorphine from a manufacturer based in Switzerland under emergency access regulations. According to interviewees, domestic production would be cheaper, as it would obviate the need to import the drug; however, this would require licensing diamorphine for treatment of OUD.
} 
iOAT currently piloted in British Columbia is pharmacy-based, intended for stabilized patients, and offers a lower-intensity treatment model (British Columbia Centre on Substance Use, 2017). In Alberta, iOAT programs have also been approved, although not yet implemented (Cameron, 2018). There is also a small residential iOAT program operating in Ottawa, Ontario ("Residential Opioid Program Giving Drug Users Chance at New Life," 2018).

In addition, RCTs evaluating HAT have been implemented in Spain, Germany, and Belgium; in Spain, HAT patients were permitted to continue the treatment following the conclusion of the trial, and HAT was legalized in Germany in 2009. In Belgium, discussions have taken place since the conclusion of the trial on the next steps (European Monitoring Centre for Drugs and Drug Addiction, 2017a). In Denmark, a HAT service opened without a trial in 2010 (European Monitoring Centre for Drugs and Drug Addiction, 2012). It also appears that Norway will start a HAT trial in the near future (Health Directorate, Norwegian Ministry of Health and Care Services, 2018; Owoseje, 2018).

\section{Evidence from the Scientific Literature}

There have been ten RCTs of HAT, the majority of which have compared supervised injectable HAT co-prescribed with flexible doses of oral methadone with oral methadone alone (Table 3.2).$^{23}$ It is important to recognize that these RCTs did not evaluate the potential for HAT to serve as a first-line treatment option or as a replacement for oral methadone. Instead, the trials have largely focused on testing the effectiveness of HAT for a particular group of treatmentrefractory individuals with a history of heroin use disorder and multiple prior attempts at conventional treatment modalities. In other words, in almost all HAT settings, participant eligibility is restricted to individuals with heroin use disorder who have tried but not responded to conventional treatments, most commonly oral methadone. Most RCTs have thus had relatively stringent participant eligibility requirements that go beyond those required to participate in conventional treatments for heroin use disorder. Although there is some variation across the trials, participants have tended to be over age 35 and male, have had a heroin use history that spans more than one decade, and have had at least three prior attempts at treatment for heroin use disorder, primarily methadone. Furthermore, in later discussion in this report, statements about HAT outperforming traditional MTs for heroin use disorder, including methadone, should be understood to mean that HAT outperformed those other treatments among people who had already tried conventional treatments. Additionally, we note that most of the evidence reviewed comes from RCTs. Although the RCT study design can offer more-rigorous causal evidence on the comparative effectiveness of HAT relative to other treatments for the eligible patient population, we recognize that there may be additional considerations and complicating factors with implementing HAT at a larger scale and outside the clinical trial setting.

\footnotetext{
${ }^{23}$ Given the shorter half-life of diamorphine relative to methadone, the adjunct of oral methadone is intended as a supplement to prevent overnight withdrawal symptoms or to provide an alternative medication should the patient be unable to attend the clinic for routine supervised doses.
} 
Table 3.2. Overview of the HAT Clinical Trials

\begin{tabular}{lccc}
\hline Source & $\begin{array}{c}\text { Approximate } \\
\text { Time Frame }\end{array}$ & Country & Treatment Sites \\
\hline Hartnoll et al. (1980) & $1972-1975$ & United Kingdom & Single clinic in London \\
Perneger (1998) & $1995-1996$ & Switzerland & Single clinic in Geneva \\
van den Brink et al. (2003) & $1998-2001$ & Netherlands & Eight treatment units in six cities \\
March et al. (2006) & $2001-2004$ & Spain & Single hospital in Granada \\
Haasen et al. (2007) & $2002-2004$ & Germany & Seven treatment centers in seven cities \\
Oviedo-Joekes et al. (2009) & $2005-2008$ & Canada & Two cities: Montreal and Vancouver \\
Strang et al. (2010) & $2005-2008$ & United Kingdom & Three clinics in England \\
Demaret et al. (2015) & $2011-2013$ & Belgium & Single clinic in Liège \\
Oviedo-Joekes et al. (2016) & $2011-2013$ & Canada & Single site in Vancouver \\
\hline
\end{tabular}

a The Dutch trial involved two treatment conditions (one injectable HAT and the other inhalable HAT) compared with oral methadone. Following other work, we treat these as two separate RCTs. For further detail on each RCT, including citations for articles reporting secondary or subgroup analyses from the RCTs, see Smart (2018).

\section{Findings for Individual-Level Outcomes}

Our review included 55 articles, spanning all ten RCTs (see Table 3.2), that met our inclusion criteria and evaluated the effectiveness of HAT with respect to patient-level outcomes. Evidence was drawn from all ten RCTs for the following outcomes: treatment retention, most commonly operationalized by studies as a binary indicator for whether a participant remained in treatment by the end of the trial; illicit or "street" heroin use; criminal offenses or criminal activities; and deaths. Evidence from most RCTs (at least seven) was available to evaluate serious adverse events related to study medication, physical or mental health, and social functioning (e.g., employment, family relationships). ${ }^{24}$ Evidence from some RCTs (fewer than seven) regarding effectiveness for alcohol use and other illicit drug use (most commonly cocaine or benzodiazepines) was also identified.

In our review of the comparative effectiveness of HAT for patient-level outcomes, outlined next, we consider the evidence base as showing strong support if all or almost all studies assessed comparative effectiveness for a given outcome, studies of comparable methodological quality did not find significant effects in opposing directions, and over two-thirds of the relevant studies found significant effects in the same direction. If this third factor did not hold, but statistically insignificant findings generally supported the same direction of the effect, we consider the evidence as suggestive.

\section{HAT Versus Oral Methadone}

Evidence from all studies indicates that HAT has benefits relative to oral methadone across several domains for persons with heroin use disorder who are refractory to traditional treatments,

\footnotetext{
${ }^{24}$ Although all RCTs evaluated outcomes related to health and social functioning, some trials (e.g., the Dutch trials) used a prespecified composite index that precluded our ability to evaluate these outcomes separately.
} 
with the strongest and most-consistent effects across studies shown for improving treatment retention and reducing illicit heroin use. As noted, these findings for HAT versus oral methadone should largely be interpreted as evidence of the relative efficacy of offering supervised injectable HAT and oral methadone, compared with offering oral methadone alone, for a patient population that has previously attempted oral methadone but continued to use heroin regularly. Trials from all countries except for the Netherlands found higher relative treatment retention rates among the HAT group, ${ }^{25}$ although differences were not significant for the relatively small Swiss $(N=51)$, Spanish $(N=62)$, or Belgian $(N=74)$ RCTs. Across all RCTs, treatment retention rates among the HAT groups are relatively high, ranging from 67 percent to more than 90 percent.

One reason HAT was more effective than methadone at treatment retention is that some participants randomly assigned to oral methadone immediately dropped out of treatment. For instance, in the German trial (Haasen et al., 2007), 28.8 percent of those assigned to oral methadone dropped out before even beginning treatment (compared with 2.3 percent of those assigned to HAT). Another is an asymmetry in the definition of retention, in that retention means remaining in "any treatment"; HAT participants can (and do) switch to methadone, but the converse was not permitted in the trials. Thus, HAT in some sense has a built-in advantage on retention measures.

There is strong evidence that HAT reduces use of illicit heroin more than oral methadone does among those who are treatment refractory. Although studies used different outcome definitions and assessment methods, all showed significantly greater reductions in illicit heroin use among the HAT trial arms. Findings from all trials also indicated that the large and significant reductions in illicit heroin use among the HAT groups occurred shortly after beginning HAT, and these lower levels of illicit heroin use persisted through the duration of the trial.

Some evidence suggests that HAT has a greater benefit than oral methadone for reducing illicit use of benzodiazepines, although this outcome was not consistently assessed across trials. Three RCTs (Swiss, German, and Belgian) found significantly greater reductions in benzodiazepine use among HAT relative to oral methadone participants. However, these effects should not be attributed to the provision of heroin alone. The Swiss trial offered clorazepate substitution treatment to participants dependent on benzodiazepines, and trial staff might have paid greater attention to reducing benzodiazepine use among participants who were receiving HAT, given concerns over potentially fatal interactions.

Evidence also suggests that HAT may be superior to oral methadone for these populations at reducing criminal activity. Across several RCTs, participants enrolled in HAT showed significantly greater reductions in illegal activities or criminal justice involvement relative to those enrolled in oral methadone. These reductions were largely driven by declines in drug offenses and property crimes, consistent with lower involvement in illicit market activities to obtain or to pay for illicit heroin. The subset of the most-recent RCTs (the UK RIOTT, Canadian

${ }^{25}$ Trial durations typically ranged from six to 12 months. 
NAOMI, and Belgian Treatment Assisted by Diacetylmorphine trials) found significant reductions in illegal activities among both the HAT and oral methadone groups but no statistically significant differences across the experimental and control conditions. It is unclear to what extent different findings across RCTs reflect differences in participant eligibility requirements, dosages for HAT or oral methadone, trial setting, or how criminal justice involvement is measured.

With respect to participant health outcomes, differences in how studies assess changes in physical and mental health create similar difficulties in synthesizing evidence for how HAT compares with oral methadone for affecting participant health outcomes. In general, trials showed that treatment-refractory participants enrolled in HAT had significant improvements in physical or mental health, although these improvements often did not differ from those observed among the control-group participants. Studies found little evidence showing a significant difference between HAT and oral methadone with respect to use of cocaine and benzodiazepines or social functioning. The lack of statistically significant effects on mortality may be attributable to the rarity of death during the trials.

Any relative benefits of HAT must be considered in light of its risks. There is strong evidence indicating that HAT carries significantly greater risk than oral methadone for serious medication-related adverse events, such as respiratory depression and seizures, although the magnitude of this risk differential varies across trials (see Smart, 2018). However, given that heroin administration in HAT is usually supervised by health care professionals and that patients are monitored following injection, the majority of serious adverse events were managed quickly, lessening the risk of more-serious consequences, such as death. Thus, while HAT may carry greater risk of adverse events than oral methadone, the delivery of pharmaceutical heroin within a supervised health care setting likely carries lower risk of adverse events than use of heroin within the illicit market context. (In this sense HAT offers some of the benefits of SCSs, with the added benefit that HAT clinics provide the heroin, so it is of known purity, whereas SCS clients have to acquire their own drugs on the street.)

\section{HAT Versus Injectable Hydromorphone}

The Canadian SALOME trial suggests that supervised injectable hydromorphone (e.g., Dilaudid) may be as effective as supervised injectable heroin for improving patient-level outcomes. Based on the results of the first six-month phase of this recent RCT $(N=202$ participants), supervised injectable heroin and supervised injectable hydromorphone showed similar effectiveness for treatment retention, illicit heroin use, illegal activities, physical health, and mental health. Additionally, the hydromorphone group in the trial had significantly fewer serious adverse events related to study medication than the HAT group. Although further study could help bolster the promising findings of this one RCT, the results already suggest that the potential importance of route of administration, as opposed to the specific provision of heroin per se. That is, the key advantage of HAT's injectable heroin over conventional oral medicationassisted treatment for treating persons who use injectable drugs may be that it is injected, not that 
it is heroin. If so, that has important implications because the U.S. Controlled Substances Act places hydromorphone in Schedule II, not Schedule I with heroin, so hydromorphone can be legally prescribed to treat pain, whereas heroin has no recognized medical application and cannot be prescribed. Nonetheless, dispensing hydromorphone to treat OUD would require regulatory adjustments.

\section{Findings for Community-Level Outcomes}

Our review identified five articles that met our inclusion criteria and evaluated the effect of HAT provision on community-level outcomes (van den Brink and Blanken, 2002; Lasnier et al., 2010; Miller et al., 2010; Ally, Brochu, and Blais, 2011; Miller et al., 2011). Studies of community-level effects did not employ an RCT research design but instead used the timing of the implementation of a HAT RCT to conduct pre-post assessments of changes in local outcomes. Two of the five identified studies analyzed community outcomes in the Canadian NAOMI context, two in the UK RIOTT context, and one in the Dutch trial context. Outcomes of interest generally included measures of public safety or public disorder, measured using police data, ethnographic or qualitative evidence, or records collected as part of the clinical trial.

Given the small number of contexts studied, methodological limitations with the research designs, differences in how community-level outcomes were measured, and the imprecision of estimated effects from the studies, we consider the evidence base for the effects of HAT on community-level outcomes to be limited. Although these studies did not find significant effects of HAT on community-level outcomes of public disorder and crime, there are three key limitations concerning their findings. First, the relevant literature is very small and included no RCTs and no quasi-experimental studies with a comparison group. Second, the trials for which community-level outcomes were studied enrolled relatively few participants, making it hard to detect adverse (or beneficial) effects at the community level; it is unclear the extent to which this evidence base would generalize to larger-scale implementation of HAT. Third, while the outcome measures studied to date provide some evidence to mitigate the concern that HAT implementation will lead to an influx of PWUO into the neighborhood (i.e., "honey pot effect"), several other community-level concerns raised about HAT (e.g., normalization of heroin use, traffic accidents, diversion of pharmaceutical heroin to illicit markets) have not yet been evaluated.

The processes by which HAT clinics operate may also determine their effects on the broader community. For instance, one of the RIOTT program's sites in South London operated out of an existing community-based alcohol and other drug use service provider's clinic, a facility that already provided methadone maintenance or other services to about 300 patients. By incorporating HAT into existing facilities, there might have been less actual or perceived impact on the community in which the clinic was located. Operating hours, capacity, accessibility, and the availability of other social services within HAT facilities may also be important factors in determining community impact. Furthermore, it is likely that HAT could generate heterogeneous community-level impacts, depending on where clinics are located. To date, all HAT facilities 
have been located in urban areas, but some have been located in cities as small as 20,000 people (Strang and Taylor, 2018). Proximity to other service providers, the existence and characteristics of local drug markets, and the preexisting socioeconomic and built environment characteristics of the communities in which HAT facilities are located may interact to generate differential impact for both patient-level and community-level outcomes.

Finally, evaluations of RCTs' impact on community-level outcomes are implicitly evaluations of HAT implemented with the procedures taken by each RCT, some of which were designed specifically to limit community impact. For instance, all RCTs have imposed some type of local residency requirement, limiting the extent to which opioid-dependent individuals might migrate to the trial cities to receive HAT. Hence, a finding in the literature that HAT has not produced a "honey pot" effect, drawing PWUO to the neighborhood in which HAT is offered, may pertain to HAT as implemented in those research trials, not HAT more generally. Overall, the ability to generalize existing evidence to allowing HAT outside an RCT context will depend on the specific design, implementation, and enforcement of allowing broader HAT availability.

\section{Findings for Economic Outcomes}

We identified seven articles, spanning five RCTs and the Swiss prospective cohort study, that examined economic outcomes related to HAT. Evidence across studies finds that supervised injectable HAT is much more expensive than oral methadone, mainly because of staffing requirements associated with supervising injections. As shown in Table 3.3, programmatic costs for HAT can be five to ten times more expensive than those for oral methadone. As demonstrated by UK policy developments, these higher total costs may become a barrier to implementation, regardless of findings on cost-effectiveness from a societal perspective. ${ }^{26}$

Table 3.3. Average Costs per Participant in Different Treatment Arms, Within-Trial Analyses from Included RCTs

\begin{tabular}{|c|c|c|c|c|c|c|c|}
\hline & \multicolumn{2}{|c|}{$\begin{array}{c}\text { Dutch (12 Months, } \\
€)\end{array}$} & \multicolumn{2}{|c|}{$\begin{array}{c}\text { NAOMI (12 Months } \\
\text { Canadian \$) }\end{array}$} & \multicolumn{3}{|c|}{$\begin{array}{c}\text { UK RIOTT (6 months, } \\
£)\end{array}$} \\
\hline & $\begin{array}{l}\text { HAT + } \\
\text { MMT }\end{array}$ & $\begin{array}{l}\text { Oral } \\
\text { MMT }\end{array}$ & $\begin{array}{l}\text { HAT + } \\
\text { MMT }\end{array}$ & Oral MMT & $\begin{array}{l}\text { HAT + } \\
\text { MMT }\end{array}$ & $\begin{array}{l}\text { Injectable } \\
\text { MMT }\end{array}$ & Oral MMT \\
\hline Program costs & 17,634 & 1,412 & 14,188 & 2,010 & 8,995 & 4,674 & 2,569 \\
\hline Drug cost & 766 & 216 & $706^{a}$ & $691^{a}$ & 1,814 & 720 & 205 \\
\hline $\begin{array}{l}\text { Other (e.g., } \\
\text { intake, } \\
\text { overhead) }\end{array}$ & 16,868 & 1,196 & $16,276^{a}$ & $3,261^{a}$ & 8,995 & 4,673 & 2,568 \\
\hline $\begin{array}{l}\text { Health care and } \\
\text { other service } \\
\text { costs }\end{array}$ & 1,160 & 1,126 & 4,586 & 6,498 & 2,190 & 1,865 & 2,023 \\
\hline
\end{tabular}

\footnotetext{
${ }^{26}$ Many of the societal benefits of drug treatment do not accrue to the agency that pays for the treatment, and treatment is like an investment, with upfront costs producing long-term benefits. Thus, expanding treatment can stress agency budgets even if they improve social welfare in the long run.
} 


\begin{tabular}{|c|c|c|c|c|c|c|c|}
\hline & \multicolumn{2}{|c|}{$\begin{array}{c}\text { Dutch (12 Months, } \\
€)\end{array}$} & \multicolumn{2}{|c|}{$\begin{array}{l}\text { NAOMI (12 Months } \\
\text { Canadian \$) }\end{array}$} & \multicolumn{3}{|c|}{$\begin{array}{c}\text { UK RIOTT (6 months, } \\
£)\end{array}$} \\
\hline & $\begin{array}{l}\text { HAT + } \\
\text { MMT }\end{array}$ & $\begin{array}{l}\text { Oral } \\
\text { MMT }\end{array}$ & $\begin{array}{l}\text { HAT + } \\
\text { MMT }\end{array}$ & Oral MMT & $\begin{array}{l}\text { HAT + } \\
\text { MMT }\end{array}$ & $\begin{array}{l}\text { Injectable } \\
\text { MMT }\end{array}$ & Oral MMT \\
\hline $\begin{array}{l}\text { Includes costs of } \\
\text { social service } \\
\text { provisions? }\end{array}$ & No & No & No & No & Yes & Yes & Yes \\
\hline $\begin{array}{l}\text { Criminal justice } \\
\text { costs }\end{array}$ & 8,756 & 12,885 & 4,893 & 6,014 & 442 & 880 & 251 \\
\hline $\begin{array}{l}\text { Includes costs of } \\
\text { corrections (e.g., } \\
\text { incarceration)? }\end{array}$ & Yes & Yes & No & No & Yes & Yes & Yes \\
\hline $\begin{array}{l}\text { Costs of crime } \\
\text { damages }\end{array}$ & 9,617 & 34,991 & 63,149 & 100,271 & 1,782 & 3,526 & 10,962 \\
\hline $\begin{array}{l}\text { Health-related } \\
\text { travel }\end{array}$ & 600 & 146 & N/A & N/A & N/A & N/A & N/A \\
\hline
\end{tabular}

NOTES: MMT = methadone maintenance treatment; N/A = not available.

a These were calculated by multiplying appendix estimates of mean monthly drug costs and mean monthly overhead costs by 12. Given actual participant dropout over the period, these are overstated. However, the within-trial estimates of program costs were not broken down by drug costs versus other, so we include these here to provide some sense of how program costs were allocated. These figures have neither been adjusted for inflation nor purchasing-power parity.

The high costs of renovating existing facilities to meet the requirements for a HAT center in Vancouver were noted as a primary factor for the nearly two-year delay in identifying a Vancouver site for the NAOMI trial (Gartry et al., 2009). However, it appears to be more costeffective in a societal sense for patients who did not do well in traditional treatment, primarily because the models credit HAT with doing more to reduce participants' levels of criminal justice involvement and associated damages to victims of their criminal activity. Most trials also showed higher quality-adjusted life years (QALYs) among the HAT arm. Among the two studies comparing the cost-effectiveness of supervised injectable heroin with other supervised medications (injectable methadone or injectable hydromorphone), there were no significant differences between injectable heroin or the other injectables in terms of costs or QALYs. ${ }^{27}$

There are several limitations to these particular findings. First, the estimates for crime costs in several cases either do not adjust for baseline crime rates or are dependent on model assumptions drawn from evidence outside the trial data. For instance, model-based estimates of cost-effectiveness for the Canadian trials used parameter estimates from a separate study (the British Columbia Methadone Maintenance Treatment Outcome Study; see Nosyk et al., 2012) and from prospective cohort studies conducted in the Netherlands and Switzerland. Second, several outcomes are omitted from the analyses. For instance, crime costs seem to have been

\footnotetext{
${ }^{27}$ The QALY construct, a measure that combines information on mortality and morbidity, is commonly used in economic evaluations of health care interventions. Briefly, it is calculated as the sum over time spent in various health states, weighted by the utility (or preference score) associated with the given health state. Within the studies identified, health-related quality of life was typically obtained from survey participants using the European Quality of Life-5 Dimensions instrument.
} 
restricted to property and violent crimes, excluding costs associated with other crimes, such as illicit drug dealing, disorderly conduct, or major traffic violations. Third, because the higher relative cost savings from HAT are driven largely by reductions in crime and scholars' estimates of how much monetary benefit to assign to the prevention of particular crimes can vary greatly (Heaton, 2010; Chalfin, 2015), results may hinge on those assumptions regarding monetizing crime prevention.

Finally, when considering expanding HAT beyond research trials, one must consider who will pay for it, a practical consideration that is perhaps of less relevance in countries with universal health care. Requiring patients to pay out of pocket would likely limit participant uptake, requirements for insurance to cover HAT costs would need to be negotiated and may prove intractable, and the likely governmental funders of HAT (health and health care agencies) are not the agencies where the bulk of savings are accrued.

\section{Legal Status in the United States}

Historically, heroin maintenance for dependent users was permissible in the United States, but the practice fell out of favor after the passage of the Harrison Act of 1914 and subsequent Supreme Court rulings (Musto, 1999). The CSA lists heroin as a Schedule I drug, which means that it is a substance with a "high potential for abuse," it has no currently accepted medical use in the United States, and there is a "lack of accepted safety" for the use of the drug under medical supervision. This classification is a significant obstacle to implementing HAT in the United States. The easiest path to implementing — or even studying - HAT would be for heroin's categorization to be amended from Schedule I to Schedule II, because Schedule II drugs can be prescribed. It might seem strange for a "street drug" to be classified as a medicine, but cocaine and methamphetamine are already in Schedule II. (Cocaine, for example, is used as a topical anesthetic and vasoconstrictor in certain types of surgery.)

As a consequence, the political and legal barriers appear to be lower for approving hydromorphone therapy than heroin, as the latter would likely need to be rescheduled should it be deployed to treat OUD. Nevertheless, scheduling is just one barrier to implementing HAT, or any new agonist therapy. Along with its schedule, a drug must be approved by the FDA for a licensed practitioner to dispense it for treatment of a "narcotic addiction." As discussed in Chapter 1, only two agonists are currently offered for such purposes, methadone and buprenorphine, meaning that hydromorphone would need to undergo additional regulatory approval.

Rescheduling can happen in two ways. First, Congress can pass a law amending the CSA to change heroin's schedule status. Second, the CSA provides explicit procedures for the Attorney General to alter the schedule placement of a drug, although this authority has been delegated to 
the administrator of the DEA (21 U.S.C. 811). ${ }^{28}$ Such rescheduling does take place from time to time, often with relatively obscure drugs (Caulkins and Coulson, 2011).

The Attorney General or DEA administrator may add, transfer, or remove a substance from the schedule after holding a hearing. This hearing may be initiated "(1) on his own motion, (2) at the request of the Secretary [of Health and Human Services], or (3) on the petition of any interested party" (21 U.S.C. 811[a]). If a hearing is held, the Secretary of Health and Human Services must provide an expert evaluation of the drug, and the recommendation of the secretary is binding as to scientific and medical matters (21 U.S.C. 811[b]). However, there is an exception for drugs that are controlled under international treaties; the DEA administrator is able to issue an order controlling the drug at issue under the schedule he or she believes is most appropriate, without regard to the procedures described above (21 U.S.C. $811[\mathrm{~d}]$ ).

The United States is a signatory of three main international treaties regarding the scheduling of drugs. HAT has been implemented in other countries that are signatories to the treaties, as the treaties allow for drug use that has medical and scientific purposes and allows "amounts which may be necessary for medical and scientific research only, including clinical trials therewith to be conducted under or subject to the direct supervision and control of the [nation]" (United Nations, 1961, Single Convention on Narcotic Drugs, art. 4, Section 1[c], art. 2, Section 5[b]). Relying on the treaties to make an argument against allowing a trial of HAT in the United States would thus be a weak position for the U.S. Department of Justice.

In theory, a state could legalize medical heroin, just as many states have legalized medical cannabis, although the probability of finding a pharmaceutical company (or other entity - e.g., a state university) to openly violate federal law and produce or import the heroin seems very slight.

Barring rescheduling, the most feasible present path toward HAT in the United States is to apply for a research exemption to conduct a study on the effectiveness of HAT. Applying for a research exemption for Schedule I drugs under the CSA has recently become easier, as the process has been moved online (DEA, undated). Originally, the U.S. Department of Health and Human Services was designated to evaluate the scientific merit of research proposals and report its findings to DEA. This responsibility was redelegated to the FDA commissioner (21 C.F.R. $5.10[\mathrm{a}][9])$, who assigned the responsibility to the controlled substances staff at the Center for Drug Evaluation and Research. These individuals now do the bulk of the evaluation of proposals, but the commissioner still makes the final decision as to whether to approve an application (FDA, 2003).

The CSA explicitly states that for Schedule I and II drugs, the

Attorney General shall register an applicant to distribute a controlled substance in schedule I or II unless he determines that the issuance of such registration is inconsistent with the public interest. In determining the public interest, the following factors shall be considered:

\footnotetext{
${ }^{28}$ In the relevant implementing regulations, the Attorney General has delegated this reclassification authority to the DEA. See 28 C.F.R. 0.100(b).
} 
(1) maintenance of effective control against diversion of particular controlled

substances into other than legitimate medical, scientific, and industrial channels;

(2) compliance with applicable State and local law;

(3) prior conviction record of applicant under Federal or State laws relating to the manufacture, distribution, or dispensing of such substances;

(4) past experience in the distribution of controlled substances; and

(5) such other factors as may be relevant to and consistent with the public health and safety. (21 U.S.C. $823[\mathrm{~b}]$; emphasis added)

The specific protocol for Schedule I drugs is also laid out in the Code of Federal Regulations. If there are defects in the application, the researcher should be notified promptly ( 21 to 30 days, depending on the type of research). If the protocol is found to not be meritorious, a researcher can request a hearing on the application (21 C.F.R. 1301.41).

\section{Insights from Interviews and Focus Groups}

Key informants were invited to comment on whether they thought that HAT could help improve outcomes for PWUO and whether HAT would be acceptable to the community. ${ }^{29}$ Table 3.4 lists all themes that emerged and their frequency (i.e., low, medium, high, or very high frequency is indicated if the theme emerged in 1-25 percent, 26-50 percent, 51-75 percent, or 76-100 percent of all transcripts, respectively) that answer these two questions. We include the indicator of frequency to show how the emergence of themes differed between professionals and PWUO. Key findings pertaining to HAT based on insights from interviews and focus groups are discussed next.

Overall, there were four key insights about HAT. First, interviewees and provider focus group participants suggested that HAT programs could potentially improve outcomes for PWUO but expressed concerns about the program enabling or perpetuating opioid use. Among the most frequently mentioned benefits of HAT were providing PWUO with a drug of known composition and that HAT may offer a suitable option for PWUO who have tried but have not succeeded with other treatments. With respect to perceived drawbacks, professionals primarily expressed concern that HAT programs would enable or perpetuate drug use - that is, facilitate the individual's use career and do little to address the underlying addiction.

\footnotetext{
${ }^{29}$ The definition of HAT used by interviewers and focus group facilitators to describe the intervention to key informants who were not familiar with HAT is listed in Chapter 2 and in the interview and study protocols in Appendixes A-C of Ober et al. (2018).
} 
Table 3.4. HAT Themes Cited by Key Informants (Professionals and PWUO)

\begin{tabular}{|c|c|c|}
\hline \multirow[b]{2}{*}{ Theme } & \multicolumn{2}{|c|}{ Frequency } \\
\hline & $\begin{array}{l}\text { Professionals }^{a} \\
\quad N=80\end{array}$ & $\begin{array}{l}\text { PWUO }^{b} \\
N=79\end{array}$ \\
\hline \multicolumn{3}{|c|}{ Could HAT Help Improve Outcomes for PWUO? } \\
\hline \multicolumn{3}{|l|}{ Reasons HAT could help improve outcomes } \\
\hline HAT programs would provide clients with a drug with known composition & Low & Medium \\
\hline HAT may be suitable for those who had tried other treatments & Low & Medium \\
\hline HAT represents another option for MT & Low & Low \\
\hline PWUO would benefit from medical supervision & Low & Low \\
\hline \multicolumn{3}{|l|}{ Reasons HAT might not help improve outcome } \\
\hline HAT programs would enable/perpetuate drug use & Low & High \\
\hline Prescription heroin would be used alongside street drugs & Low & High \\
\hline Gaps in HAT provision (e.g., because of insurance lapses) would be problematic & Low & Medium \\
\hline PWUO may not be trusting of a HAT program & Low & N/A \\
\hline HAT may give rise to staff safety issues & Low & Low \\
\hline \multicolumn{3}{|l|}{ Not sure whether HAT would help improve outcomes } \\
\hline Need to see evidence to comment on effectiveness & Medium & N/A \\
\hline \multicolumn{3}{|l|}{ Would HAT Be Acceptable to the Community? } \\
\hline HAT implementation would be impeded by community values and local culture & High & Low \\
\hline Community would think that HAT enables or perpetuates use & Low & Low \\
\hline Community members would be concerned about neighborhood effects & Low & Low \\
\hline $\begin{array}{l}\text { Community members would be open to HAT as an extension of existing MT } \\
\text { options }\end{array}$ & Low & Low \\
\hline Community members would be concerned about diversion of prescription heroin & Low & N/A \\
\hline $\begin{array}{l}\text { Community would be in favor of providing PWUO with a drug with known } \\
\text { composition }\end{array}$ & Low & N/A \\
\hline PWUO face stigmatization in their communities & Low & Low \\
\hline Community members would be reluctant to provide funding & Low & Low \\
\hline
\end{tabular}

NOTES: N/A: Not applicable; theme did not arise in any transcripts; Low frequency: Theme arose in 1-25\% of transcripts; Medium frequency: Theme arose in 26-50\% of transcripts; High frequency: Theme arose in 51-75\% of transcripts; Very high frequency: Theme arose in $76-100 \%$ of transcripts.

a Professionals include all non-PWUO key informants who participated in an interview $(N=44)$ or focus group ( $N=5$ groups and 36 providers) who were invited to participate based on their current profession. We are mindful that at least some of consulted PWUO may also be professionals. The use of professional in this report is not a comment on any individual's job status; rather, it is meant to capture the fact that we invited one group of key informants to inform the research project on the grounds of their occupations.

${ }^{b}$ PWUO who participated in ten focus groups.

Second, PWUO currently engaged with treatment services for their opioid use were more skeptical of the effectiveness of HAT than other key informants. In PWUO focus groups, concerns were expressed that HAT would enable or perpetuate drug use and worried that street drugs would continue to be used alongside HAT. PWUO who were actively using opioids and not currently in treatment were the most open to HAT, particularly with respect to the fact that HAT clients would be prescribed a safe drug, although these PWUO also identified a series of potential drawbacks. PWUO, in particular, along with some other key informants, also expressed concern that any gaps in continuity caused by capacity or insurance issues could result in being dropped from treatment and left with an active addiction, similar to the experience many had with prescription opioids.

Third, despite potential benefits for some PWUO, key informants suggested that HAT implementation might not be feasible because of practical and legal concerns and potentially to community resistance. The most frequently cited reason that HAT likely would be difficult to 
implement was the belief that potential implementation would be impeded by local community values and culture, as well as stigma toward PWUO, impediments that have been common in attempting to establish SEPs and other programs for PWUO. Other, albeit less frequently mentioned, obstacles to HAT acceptability included local opposition around siting of the program (referred to by key informants as "NIMBYism," reflecting the acronym for the colloquialism "not in my backyard"), as well as concerns surrounding the diversion of prescribed heroin.

Fourth, making evidence on HAT effectiveness available to community stakeholders is considered critical to any debate surrounding HAT. Approximately a third of interviewees expressed desire to learn about the evidence underlying HAT programs. Other key informants suggested that community education about HAT and the evidence of its effectiveness (to the extent that it exists) would be required to even begin the argument for the implementation of HAT and to influence the corresponding policy debate. A related observation made by some interviewees was that emphasizing the medical nature of HAT may be effective in addressing some concerns that community stakeholders may have. Several interviewees pointed out that a small-scale pilot program may be useful to demonstrate how a HAT service operates and examine whether it can be effective in improving outcomes for PWUO and whether it is acceptable to the community. 


\section{Supervised Consumption Sites}

\section{International Landscape}

SCSs (sometimes known as safe injection facilities, drug consumption rooms, or overdose prevention sites) are facilities that aim to reduce the acute and chronic harms from drug use. They are characterized as a low-threshold intervention, ${ }^{30}$ intended to attract individuals facing high risk of drug-related harms (e.g., people who inject drugs, sex workers who use drugs, those who are homeless). SCSs initially focused on reducing the spread of HIV/AIDS, hepatitis C, and other health and social consequences of unhygienic injection drug use (Hedrich, 2004; European Monitoring Centre for Drugs and Drug Addiction, 2017b). Because SCSs permit drug users to consume illicitly obtained substances under trained supervision, they also aim to avert fatal drug overdoses and reduce externalities associated with public drug use. ${ }^{31}$ Most SCSs still focus on injection drug use, although some in Europe and Canada allow or include space for smoking crack cocaine and other drugs. SCSs also may or may not be integrated with broader public health and treatment services and vary considerably across countries in form and range of services provided.

Spending taxpayer funds to facilitate consumption of illegal drugs strikes some as incongruous, particularly on first hearing, but the logic of SCSs can, in a way, be seen as paralleling familiar medical interventions, such as chemotherapy and kidney dialysis. Patients receive treatment in outpatient facilities designed for the purposes of transfusing medicines and fluids under staff supervision in a sterile setting, rather than risk error or harm by letting patients attempt such procedures at home on their own. Individuals who inject street-sourced drugs, and who may be homeless, can benefit from such a sterile and safe environment. ${ }^{32}$ Further, the increased risk of fatal overdose from the proliferation of potent synthetic opioids makes supervising drug consumption even more relevant today. However, questions remain surrounding the magnitude of the effectiveness and cost-effectiveness of supervised drug consumption under an SCS model.

According to the European Monitoring Centre for Drugs and Drug Addiction (2017b), SCSs traditionally "represent a local response, closely linked to policy choices made by local

\footnotetext{
30 The European Centre for Disease Prevention and Control and the European Monitoring Centre for Drugs and Drug Addiction (2011) describe low-threshold services as social and health services that aim to reach as many problematic drug users as early as possible in their drug-using careers and to remain in contact with such individuals to prevent health damage while they continue to use drugs.

${ }^{31}$ Like SEPs, SCSs seek to reduce disease transmission caused by use of unhygienic needles and to connect highrisk populations with social services. An increasing number of SEPs are also distributing naloxone. Indeed, Wheeler et al. (2015) noted that SEPs were some of the early adopters providing naloxone to PWUO.

${ }^{32}$ For more information on this logic model, see Pardo, Caulkins, and Kilmer (2018), which builds on the work of the European Monitoring Centre for Drugs and Drug Addiction (2017a).
} 
stakeholders, based on an evaluation of local need and determined by municipal or regional options." Therefore, they employ a user-oriented service model, sometimes offering a wide array of additional services, including education, access to basic health care, psychosocial counseling, and sometimes testing of drugs for adulterants.

The first SCS opened in Bern, Switzerland, in 1986 amid a policy shift that emphasized survival and low-threshold services that attract the most at-risk populations (Hedrich, 2004). The objectives were twofold: to reduce health harms, including the number of overdoses and the incidence of new HIV infections, and to reduce the nuisance created by public drug use. SCSs slowly expanded to other cities in Switzerland. By the mid-1990s, cities in Germany and the Netherlands started to implement SCSs.

Today, there are some 90 facilities operating in nine countries in Europe, and policymakers in Ireland are planning to open a facility in Dublin (Brophy, 2018). Outside Europe, there is a growing number of facilities in Canada and Australia. ${ }^{33}$ Discussions of opening SCSs have started in other cities in North America, such as Denver, Philadelphia, and San Francisco (Brown and Murphy, 2017; Lopez, 2018). Table 4.1 shows the number of cities and approved SCSs reported to be in operation, by country. Until 2016, Vancouver was the only site in North America allowed to operate an SCS, although others are reported to have operated without official approval for some time (Kral and Davidson, 2017). Because overdose deaths continued to increase in 2017, the Canadian government has permitted additional fixed and mobile sites to operate (Wherry, 2017).

Interviews with international key informants noted that there was often vocal opposition to SCSs when initially discussed or opened. This opposition revolved primarily around concerns about enabling drug use and potential negative community effects. However, according to interviewees, objections to SCSs from local stakeholders tended to disappear following their implementation, something that was also observed in numerous places that opened SEPs (Tempalski, 2007). As confirmed by international interviewees in Switzerland, the Netherlands, and British Columbia, SCSs are generally accepted services for people with OUD.

Table 4.1. Supervised Consumption Sites Around the World

\begin{tabular}{lcc}
\hline Country & Number of Cities with SCSs & Number of SCSs Operating \\
\hline Netherlands & 25 & 31 \\
Germany & 15 & 24 \\
Canada & $11(5$ more planned $)$ & $20^{\mathrm{b}}(11$ more planned $)$ \\
Switzerland & 8 & 12 \\
Spain & 7 & 13 \\
Denmark & 4 & 5
\end{tabular}

33 As this report was going to press, it was reported that an SCS opened in Mexicali, near the Mexico-U.S. border (Romero, 2018). 


\begin{tabular}{lcc}
\hline Country & Number of Cities with SCSs & Number of SCSs Operating \\
\hline Norway & 2 & 2 \\
France & 2 & 2 \\
Australia & 2 & 2 \\
Luxembourg & 1 & 1 (1 more planned) \\
Ireland & 1 planned & 1 planned \\
\hline
\end{tabular}

SOURCES: European Monitoring Centre for Drugs and Drug Addiction, 2017b; Health Canada, 2018.

a Until 2017, there were only two operational SCSs in Canada.

${ }^{\mathrm{b}}$ British Columbia has also deployed low-threshold SCSs in the form of "overdose prevention sites." This count does not include such sites, which aim to reduce overdoses by allowing social workers and other injection drug users to set up facilities on the street to monitor injection drug use and distribute or administer naloxone.

\section{Evidence from Scientific Literature}

The existing reviews of scientific evaluations of SCSs report positive findings across a broad range of outcomes (Kerr et al., 2007; McNeil and Small, 2014; Potier et al., 2014; Garcia, 2015; Kennedy, Karamouzian, and Kerr, 2017). ${ }^{34}$ In general, the studies evaluated public and SCS client opinions, individual-level outcomes (e.g., access or referral to treatment, changes in drug use practices, risky drug use, social and health outreach), and other outcomes (e.g., morbidity and mortality, crime, public disorder). However, the majority of studies come from a handful of sites; almost 80 percent of the literature base evaluated in systematic reviews comes from Insite in Vancouver or the Medically Supervised Injection Centre (MSIC) in Sydney.

We conducted our own assessment of the individual studies and found that the evidence base concerning the overall effects of SCSs is limited in quality and location. Although we identified 65 outcome-related articles (as opposed to commentaries or studies that gauge opinions), none involved an RCT, and just nine employed a quasi-experimental design with control groups. These nine studies were based on just four SCSs in three cities: Sydney (five studies), Vancouver (two studies), and Barcelona (two studies). There was considerable overlap in the design, methods, authorship, and data employed, so that four of the nine are arguably superseded by later studies using better methods or longer time series, in some sense reducing the effective number of quasi-experimental studies to five.

\footnotetext{
${ }^{34} \mathrm{We}$ also considered one grey literature review that does not detail selection criteria nor report study findings systematically. Rather, that literature review "aims to provide a descriptive analysis of historical background, operational frameworks and outcomes" related to SCSs (Hedrich, 2004). The review focuses largely on individual health outcomes, as well as community-level outcomes, such as crime and public drug use. In most cases, Hedrich (2004) did not discuss study design of articles, although most appear to be descriptive survey-based studies targeted at SCS clients and staff. Findings reported by Hedrich (2004) provide an additional level of detail not captured elsewhere.

While we were writing this report, a meta-analysis of SCSs (May, Bennett, and Holloway, 2018) was published, but it was then retracted because of the "methodological weaknesses linked to the pooling of diverse outcomes into a single composite measure" (International Journal of Drug Policy, 2018). It should be noted that the study was not retracted for its selection criteria, which identified a small number of high-quality studies about SCSs.
} 
Unlike these five, most of the studies included in the SCS literature reviews employ methodologies that do not allow for making causal inferences. For example, they might not include control groups or might compare frequent SCS users with those who use the sites less frequently. These studies can provide information about typical clients or costs, but they are not well suited to teasing out the effects of SCSs on individual or population-level outcomes.

In the next subsections, we describe our review of the quasi-experimental studies, as well as those that employ mathematical simulation or modeling. Pardo, Caulkins, and Kilmer (2018) provided additional information about the 65 studies.

\section{Quasi-Experimental Studies}

The nine studies employing a quasi-experimental design examined outcomes related to overdose, discarded injection equipment, or crime (Table 4.2). We are not aware of any quasiexperimental studies examining the effect of SCSs on treatment uptake or other health outcomes. The studies used varying analytical approaches. Some earlier studies merely visually compared outcomes between treatment and control cases; later studies included statistical tests.

Table 4.2. Quasi-Experimental Evaluations of Supervised Consumption Sites

\begin{tabular}{|c|c|c|c|c|c|c|c|}
\hline Study & $\begin{array}{l}\text { Outcomes } \\
\text { Measured }\end{array}$ & Period & Location & $\begin{array}{l}\text { Analytical } \\
\text { Method }\end{array}$ & $\begin{array}{l}\text { Treatment } \\
\text { Designation }\end{array}$ & $\begin{array}{c}\text { Control } \\
\text { Designation }\end{array}$ & $\begin{array}{l}\text { SCS Association } \\
\text { with Outcome }\end{array}$ \\
\hline $\begin{array}{l}\text { Freeman } \\
\text { et al. (2005) }\end{array}$ & $\begin{array}{l}\text { Incidence } \\
\text { of drug- } \\
\text { related } \\
\text { property } \\
\text { and violent } \\
\text { crime }\end{array}$ & $\begin{array}{l}\text { September } \\
1999- \\
\text { October } \\
2002\end{array}$ & Sydney & $\begin{array}{l}\text { Visual } \\
\text { comparison } \\
\text { across cases; } \\
\text { time series } \\
\text { analysis with a } \\
\text { segmented } \\
\text { regression } \\
\text { approach } \\
\text { within } \\
\text { treatment case }\end{array}$ & $\begin{array}{l}\text { Local area } \\
\text { command } \\
\text { where SCS is } \\
\text { located-Kings } \\
\text { Cross } \\
\text { neighborhood }\end{array}$ & $\begin{array}{l}\text { Rest of } \\
\text { Sydney }\end{array}$ & $\begin{array}{l}\text { No visual difference in } \\
\text { reported crime trends in } \\
\text { treatment and control } \\
\text { areas; regression } \\
\text { coefficient indicating } \\
\text { operation of SCS not } \\
\text { statistically significant } \\
\text { with incidence of crime } \\
\text { reported in treatment } \\
\text { area }\end{array}$ \\
\hline $\begin{array}{l}\text { National } \\
\text { Centre in } \\
\text { HIV } \\
\text { Epidemi- } \\
\text { ology and } \\
\text { Clinical } \\
\text { Research } \\
(2007)\end{array}$ & $\begin{array}{l}\text { Fatal } \\
\text { overdoses, } \\
\text { overdose- } \\
\text { initiated } \\
\text { emergency } \\
\text { service } \\
\text { calls, and } \\
\text { emergency } \\
\text { department } \\
\text { episodes } \\
\text { for opioids }\end{array}$ & $\begin{array}{l}\text { May 1998- } \\
\text { April } 2006\end{array}$ & Sydney & $\begin{array}{l}\text { Poisson } \\
\text { regression to } \\
\text { test } \\
\text { significance } \\
\text { between } \\
\text { treatment and } \\
\text { control cases }\end{array}$ & $\begin{array}{l}\text { Postal zone } \\
\text { where SCS is } \\
\text { located in } \\
\text { Kings Cross } \\
\text { neighborhood }\end{array}$ & $\begin{array}{l}\text { Rest of state } \\
\text { of New South } \\
\text { Wales }\end{array}$ & $\begin{array}{l}\text { No significant } \\
\text { difference in number of } \\
\text { fatal overdoses } \\
\text { between treatment and } \\
\text { control areas; } \\
\text { significant difference in } \\
\text { decline of emergency } \\
\text { service calls in } \\
\text { designated treatment } \\
\text { area relative to rest of } \\
\text { state }\end{array}$ \\
\hline $\begin{array}{l}\text { Fitzgerald, } \\
\text { Burgess, } \\
\text { and } \\
\text { Snowball } \\
(2010)\end{array}$ & $\begin{array}{l}\text { Criminal } \\
\text { incident } \\
\text { reports of } \\
\text { robbery, } \\
\text { theft, and } \\
\text { drug- } \\
\text { specific } \\
\text { crime }\end{array}$ & $\begin{array}{l}\text { January } \\
\text { 1999- } \\
\text { March } 2010\end{array}$ & Sydney & $\begin{array}{l}\text { Visual } \\
\text { comparison } \\
\text { across cases; } \\
\text { nonparametric } \\
\text { hypothesis test } \\
\text { (tau coefficient) } \\
\text { to test within- } \\
\text { case changes }\end{array}$ & $\begin{array}{l}\text { Local area } \\
\text { command } \\
\text { where SCS is } \\
\text { located-Kings } \\
\text { Cross } \\
\text { neighborhood }\end{array}$ & $\begin{array}{l}\text { Rest of } \\
\text { Sydney }\end{array}$ & $\begin{array}{l}\text { No visual difference in } \\
\text { reported theft and } \\
\text { robbery incidents } \\
\text { between treatment and } \\
\text { control case areas; } \\
\text { drug-specific crime } \\
\text { stable in treatment } \\
\text { area, up in rest of } \\
\text { Sydney; significant } \\
\text { reductions of robbery } \\
\text { and crime within cases } \\
\text { over time }\end{array}$ \\
\hline
\end{tabular}




\begin{tabular}{|c|c|c|c|c|c|c|c|}
\hline Study & $\begin{array}{l}\text { Outcomes } \\
\text { Measured }\end{array}$ & Period & Location & $\begin{array}{l}\text { Analytical } \\
\text { Method }\end{array}$ & $\begin{array}{l}\text { Treatment } \\
\text { Designation }\end{array}$ & $\begin{array}{c}\text { Control } \\
\text { Designation }\end{array}$ & $\begin{array}{l}\text { SCS Association } \\
\text { with Outcome }\end{array}$ \\
\hline $\begin{array}{l}\text { Salmon et al. } \\
(2010)\end{array}$ & $\begin{array}{l}\text { Emergency } \\
\text { service } \\
\text { calls for } \\
\text { opioid- } \\
\text { related } \\
\text { overdoses }\end{array}$ & $\begin{array}{l}\text { May } 1998- \\
\text { May } 2006\end{array}$ & Sydney & $\begin{array}{l}\text { Poisson } \\
\text { regression to } \\
\text { test } \\
\text { significance } \\
\text { between } \\
\text { treatment and } \\
\text { control cases }\end{array}$ & $\begin{array}{l}\text { Where SCS is } \\
\text { located in } \\
\text { Kings Cross } \\
\text { neighborhood; } \\
\text { postal zone } \\
\text { where SCS is } \\
\text { located }\end{array}$ & $\begin{array}{l}\text { Rest of State } \\
\text { of New South } \\
\text { Wales }\end{array}$ & $\begin{array}{l}\text { Significant difference in } \\
\text { decline of emergency } \\
\text { service calls in } \\
\text { designated treatment } \\
\text { area relative to rest of } \\
\text { state }\end{array}$ \\
\hline $\begin{array}{l}\text { Marshall et } \\
\text { al. }(2011)\end{array}$ & $\begin{array}{l}\text { Fatal drug } \\
\text { overdoses }\end{array}$ & $\begin{array}{l}\text { January } \\
2001- \\
\text { December } \\
2005\end{array}$ & Vancouver & $\begin{array}{l}\text { Nonparametric } \\
\text { hypothesis test } \\
\text { (Wilcoxon test); } \\
\text { nonlinear } \\
\text { modeling of } \\
\text { rate difference } \\
\text { pre-post }\end{array}$ & $\begin{array}{l}500 \text { meter } \\
\text { radius around } \\
\text { SCS }\end{array}$ & $\begin{array}{l}\text { Blocks } 500- \\
\text { meter radius } \\
\text { outside SCS }\end{array}$ & $\begin{array}{l}\text { Significant reductions in } \\
\text { the number of } \\
\text { overdoses within } \\
\text { treatment area } \\
\text { compared with rest of } \\
\text { city; reductions in } \\
\text { overdose rate in } \\
\text { person-years } \\
\text { exponentially declines } \\
\text { farther from SCS }\end{array}$ \\
\hline $\begin{array}{l}\text { Donnelly } \\
\text { and } \\
\text { Mahoney } \\
\text { (2013) }\end{array}$ & $\begin{array}{l}\text { Criminal } \\
\text { incident } \\
\text { reports and } \\
\text { rates of } \\
\text { robbery, } \\
\text { theft, and } \\
\text { drug- } \\
\text { specific } \\
\text { crime }\end{array}$ & $\begin{array}{l}\text { January } \\
\text { 1999- } \\
\text { December } \\
2012\end{array}$ & Sydney & $\begin{array}{l}\text { Visual } \\
\text { comparison } \\
\text { across cases; } \\
\text { nonparametric } \\
\text { hypothesis test } \\
\text { (tau coefficient) } \\
\text { to test within- } \\
\text { case changes }\end{array}$ & $\begin{array}{l}\text { Local area } \\
\text { command } \\
\text { where SCS is } \\
\text { located-Kings } \\
\text { Cross } \\
\text { neighborhood }\end{array}$ & $\begin{array}{l}\text { Rest of } \\
\text { Sydney }\end{array}$ & $\begin{array}{l}\text { No visual difference in } \\
\text { reported crime trends } \\
\text { incidents between } \\
\text { treatment and control } \\
\text { case areas; significant } \\
\text { reductions of crime } \\
\text { within cases over time }\end{array}$ \\
\hline $\begin{array}{l}\text { Vecino et al. } \\
(2013)\end{array}$ & $\begin{array}{l}\text { Discarded } \\
\text { injection } \\
\text { material }\end{array}$ & $2004-2012$ & Barcelona & $\begin{array}{l}\text { Nonparametric } \\
\text { hypothesis test } \\
\text { (Mann- } \\
\text { Whitney) }\end{array}$ & $\begin{array}{l}\text { Two districts } \\
\text { where SCSs } \\
\text { opened }\end{array}$ & $\begin{array}{l}\text { Rest of } \\
\text { Barcelona }\end{array}$ & $\begin{array}{l}\text { No significant change } \\
\text { in number of improperly } \\
\text { discarded syringes in } \\
\text { treatment district; } \\
\text { statistically significant } \\
\text { reduction of discarded } \\
\text { injection equipment } \\
\text { across city }\end{array}$ \\
\hline $\begin{array}{l}\text { Espelt et al. } \\
(2017)\end{array}$ & $\begin{array}{l}\text { Discarded } \\
\text { injection } \\
\text { material }\end{array}$ & 2004-2014 & Barcelona & $\begin{array}{l}\text { Interrupted } \\
\text { time series, } \\
\text { Poisson } \\
\text { regression }\end{array}$ & $\begin{array}{l}\text { Districts B and } \\
\text { C where SCS } \\
\text { opened }\end{array}$ & $\begin{array}{l}\text { Nontreatment } \\
\text { districts in } \\
\text { city }\end{array}$ & $\begin{array}{l}\text { Short-term increase in } \\
\text { discarded syringes in } \\
\text { treatment and control } \\
\text { districts, then decrease } \\
\text { and stabilization in } \\
\text { number of improperly } \\
\text { discarded syringes in } \\
\text { public spaces across } \\
\text { city }\end{array}$ \\
\hline $\begin{array}{l}\text { Myer and } \\
\text { Belisle } \\
(2018)\end{array}$ & $\begin{array}{l}\text { Reported } \\
\text { incidence } \\
\text { of violent } \\
\text { and } \\
\text { property } \\
\text { crime }\end{array}$ & $\begin{array}{l}\text { January } \\
2002- \\
\text { December } \\
2004\end{array}$ & Vancouver & $\begin{array}{l}\text { Time series } \\
\text { analysis using } \\
\text { autoregressive } \\
\text { integrated } \\
\text { moving } \\
\text { average to } \\
\text { compare } \\
\text { changes over } \\
\text { time within } \\
\text { cases }\end{array}$ & $\begin{array}{l}\text { Police District } \\
1 \text {, where SCS } \\
\text { is located }\end{array}$ & $\begin{array}{l}\text { Police } \\
\text { Districts 2-4 }\end{array}$ & $\begin{array}{l}\text { Significant reductions in } \\
\text { reported property and } \\
\text { violent crime in } \\
\text { treatment district after } \\
\text { SCS opening; no } \\
\text { reported reductions in } \\
\text { crime in control districts }\end{array}$ \\
\hline
\end{tabular}

The comparison areas in these quasi-experimental studies are not always ideal. Preferably, quasi-experimental evaluations will select control areas that are reasonably similar to the treatment area to reduce the potential for confounding effects. The purpose of comparing trends in the treatment area with those in a control region is to allow one to subtract out general trends 
over time that are caused by exogenous factors, not the intervention being evaluated (this is known as a differences-in-differences approach).

Six of the nine studies use either the whole city or even the entire state as a control case when examining outcomes in the treatment area immediately surrounding the SCS (Donnelly and Mahoney, 2013; Fitzgerald, Burgess, and Snowball, 2010; Freeman et al., 2005; National Centre in HIV Epidemiology and Clinical Research, 2007; Salmon et al., 2010; Vecino et al., 2013). Because SCSs are intentionally sited in areas with high rates of injection drug use, they are usually different from the city or state as a whole. Three of the studies do use as controls some other specific districts within the city, or, in one case, outside a 500-meter radius from the SCS (Espelt et al., 2017; Myer and Belisle, 2018; Marshall et al., 2011), potentially reducing the risk of factors other than the treatment intervention accounting for any observed differences between the treatment and control areas.

Furthermore, the quasi-experimental studies were not all independent. In some cases, subsequent studies examined the same intervention and outcome variables, just with a longer time series and (sometimes) more-sophisticated methods. For example, the three studies examining crime outcomes of the MSIC in Sydney were very similar (Donnelly and Mahoney, 2013; Fitzgerald, Burgess, and Snowball, 2010; Freeman et al., 2005). They all reported that changes in thefts, robberies, and drug-law violations where the SCS was located were similar to changes in the rest of the city, suggesting a null effect.

In contrast, the study from Vancouver of the SCS's effect on crime used a more rigorous analytical design that accounted for underlying trends and seasonality (Myer and Belisle, 2018). It found that there was an abrupt and permanent decline in property and violent crime in the police district where the SCS was located vis-à-vis elsewhere in the city. That is, in some sense, a surprising result. Merely supervising drug use might not be expected to reduce the quantity of drugs used or the quantity of drugs purchased or sold, and those are the activities that can stimulate crime - for example, property crime committed to finance a drug purchase.

Supervising drug use is intended to reduce overdose and the spread of blood-borne diseases, but neither of those outcomes directly stimulates crime. It may be that services provided by Vancouver's SCS facility besides the supervision of consumption, such as treatment referral, aid in reducing acquisitive property crime. No study reported an increase in crime associated with SCS operation.

Two overlapping studies from Sydney that examined overdose-involved outcomes found a statistically significant negative relationship with ambulance service calls for suspected opioidinvolved overdoses (National Centre in HIV Epidemiology and Clinical Research, 2007; Salmon et al., 2010). Only the earlier study examined fatal overdoses, and it did not find a statistically significant effect. As mentioned, the SCS in question opened around the same time there was a severe shortage of heroin in Australia's drug markets (Weatherburn et al., 2002), so opioid overdose-involved outcomes fell precipitously in the control areas and even more precipitously near the SCS. That control area was the entire state of New South Wales, so it was not very similar to the treatment area. Therefore, it is plausible that an exogenous shock as dramatic as the 
Australian heroin drought might have affected the SCS's neighborhood more strongly than it affected other parts of the state. However, the measured effect appeared strongest during the facility's hours of operation, which would be consistent with the SCS causing additional declines in overdose above and beyond that produced statewide by the heroin drought.

The Vancouver study of overdose outcomes reported even more favorable SCS effects (Marshall et al., 2011). Although overdose fell somewhat in the control areas, declines around the time and place the SCS opened were much greater, with the rate of decline in fatal overdoses falling with greater distance from the facility.

The remaining quasi-experimental studies analyzed SCSs in Barcelona and are very similar to each other (Vecino et al., 2013; Espelt et al., 2017), although the latter applied a more rigorous research design. Results from these two studies are somewhat inconsistent. Vecino et al. (2013) found no significant change in number of improperly discarded syringes in the treatment district but did report a statistically significant reduction of discarded injection equipment across the city. Espelt et al. (2017) extended Vecino et al.'s (2013) analysis by using an interrupted time series with Poisson regression and additional control covariates. After an SCS was opened in the Ciutat Vella district, the authors reported a modest but statistically significant reduction in the number of publicly disposed injection materials in the area but no changes in neighboring districts. After an SCS opened in Sant Martí, they reported findings that show a statistically significant and substantial increase in publicly disposed injection material. The authors noted that simultaneous police sweeps focused on public drug use might have confounded their analyses.

\section{Mathematical Simulations}

A variety of studies use mathematical models to extrapolate from proximate evaluation outcomes to overall effects, often within a cost-effectiveness or benefit-cost framework. Just as we urge caution when interpreting the quasi-experimental studies on SCS outcomes, we suggest the same about the eight studies labeled as "mathematical simulations" in Kennedy, Karamouzian, and Kerr's (2017) review. ${ }^{35}$

Only one was a simulation study in the sense of explicitly simulating the evolution of the state of some system (such as the HIV epidemic), both with and without the SCS (Bayoumi and Zaric, 2008), and that study has been criticized as producing implausible results (Des Jarlais, Arasteh, and Hagan, 2008; Pinkerton, 2010; Andresen and Jozaghi, 2012). The others primarily used Monte Carlo simulation to explore how uncertainty about parameter values could have affected outcomes that were computed from a deterministic model.

With the exception of Hedrich (2004), which was not really a simulation but just assumed that SCSs cut the mortality risk from 0.02 per 1,000 use sessions to zero, and Jozaghi (2014),

\footnotetext{
35 There are other simulation studies that project what the benefits might be of opening SCSs in places where they do not exist. We do not discuss them here.
} 
which examined a small, unsanctioned supervised smoking facility for crack users, these studies all pertain to the Insite facility in Vancouver.

Milloy et al. (2008) estimated the number of fatal overdoses averted by dividing the number of near-fatal overdoses within the SCS by an assumed ratio of near-fatal to fatal overdoses. That ratio is unknown, with plausible values differing by a factor of six, so the resulting point estimates vary from 1.9 to 11.7 deaths averted per year, and uncertainty about other parameters renders those point estimates into ranges - for example, the 11.7 comes with a plausible range from 5.4 to 18.0 .

The four studies modeling Insite's effects on HIV transmission represent two camps that disagree. Andresen and Boyd (2010) and Andresen and Jozaghi (2012) assumed that the SCS causes people who perform all, most, or some of their injections at the site to also behave much more safely when they inject outside the SCS, and their articles credit Insite's SCS with averting 35 and 22 new HIV infections per year, respectively.

Pinkerton $(2010,2011)$ is sharply critical of Andresen and Boyd, particularly of the models (which indeed seem to have some errors; described in more detail in Pardo et al., 2018) and the assumption that consuming some drugs at Insite also alters the way those individuals use drugs outside Insite's walls. Pinkerton's 2010 and 2011 articles take a more conservative approach and credit the SCS side of Insite's suite of programs with averting only 2.8 and 5.2 new HIV infections per year, respectively, although Pinkerton credits Insite's distribution of injection equipment for use outside Insite (its SEP component) with preventing more HIV infections.

This crucial disagreement about behavioral effects boils down to how one interprets findings, such as those reported by Kerr et al. (2005). Based on a logistic regression, Kerr et al. (2005) found that those who reported using the SCS for all, most, or some of their injections were 70 percent less likely to have reported having shared injection equipment than other users. Pinkerton (2011) noted that Kerr et al. reported a difference in the proportion of people who have shared, not the number of instances of sharing. It is also not certain that Insite caused the observed differences, as Kerr et al. admit. Furthermore, even if Insite did cause behavioral change outside its walls, it is not clear how one could know that Insite's supervising of drug consumption should get the credit, as opposed to its SEP, referrals to treatment, or some other component of its multifaceted operation.

This disagreement has implications for Insite's cost-effectiveness in terms of HIV/AIDS prevention. The majority of the modeling studies assume a benefit of C $\$ 210,000$ (Canadian dollars) per HIV infection prevented from lifetime averted health care costs. Using Pinkerton's estimates of 2.8 and 5.2 new HIV infections averted per year as a result of Insite's SCS function, this translates into savings of about $\mathrm{C} \$ 0.6$ million or $\mathrm{C} \$ 1.1$ million per year. That is less than Insite's cost of supervising consumption, which the author describes as C \$1.5 million of Insite's approximately C\$3 million annual budget. ${ }^{36}$ Pinkerton argues for cost-effectiveness primarily

\footnotetext{
${ }^{36}$ It is unclear to what extent this includes fixed costs. Andresen and Boyd (2010, p. 71) noted:
} 
based on Insite's SEP, not its SCS. By contrast, valuing Andresen and Jozaghi's (2012) estimated $22 \mathrm{HIV}$ cases averted per year at C\$210,000 per case produces a benefit of C\$4.6 million, well in excess of the cost of operating Insite's SCS and, for that matter, all of Insite.

However, the question for policymakers is not necessarily whether to invest in SCS or do nothing but rather how much of scarce funds should be invested in SCS, compared with other alternatives, because supervising drug consumption in a facility such as Insite may be costly. Pinkerton $(2010,2011)$ credits Insite with supervising 220,000 injections per year, although 2017 data indicate a figure closer to 150,000 (Vancouver Coastal Health, 2018). In round terms, dividing Insite's C\$1.5 million in annual SCS costs over roughly 200,000 supervised injections suggests an average cost of approximately C\$7.50 per injection, and PWUO often inject multiple times per day.

For someone who averages two injections at Insite per day, monthly and annual costs would be $C \$ 450$ and $C \$ 5,500$, respectively. We are not aware of any studies that formally compare the costs associated with SCSs and treatment; however, these back-of-the-envelope calculations suggest that the costs of supervising consumption of a full month's worth of use might be in the same ballpark as the costs of providing methadone in the United States for the same duration, ${ }^{37}$ although obtaining precise figures for treatment is surprisingly tricky because costs vary

The annual operational cost (2007) of the SIF [supervised injection facility] portion of Insite has been cited as \$1.5 million (CTV News, 2008, an interview of Dr. Thomas Kerr, Principal Investigator, Insite). Operational costs of Insite have also been described as \$2 million (CBC News, 2003) and \$3 million (Health Canada, 2008), but these figures included such other services as addiction counselling and case management, the provision of primary healthcare, public health screening (immunizations and diagnostics), addiction and housing services, education, and peer counselling. We use the $\$ 1.5$ million figure for two reasons: first, it only considers the operational costs of the SIF portion of Insite; and second, the source is the Principal Investigator contracted by Health Canada to evaluate Insite.

${ }^{37}$ For example, the Washington State Institute for Public Policy (2017) estimated that the annual per-participant costs for methadone were $\$ 3,769$ (in 2016 dollars):

We estimate the per-participant costs of providing methadone in addition to standard substance abuse treatment for 12 months. Costs reflect the average of costs reported in numerous costeffectiveness studies (Rosenhack and Kosten, 2001; Jones et al., 2009; Nordlund et al., 2004; Masson et al, 2004). Costs included vary by study but generally include costs of medication, dispensing, toxicology screens, medical care related to methadone treatment, and when available, costs of equipment, administration, and clinic space. Treatment as usual in this case may include counseling or other services.

The National Institute on Drug Abuse (2018b) reported that, although the price for opioid treatment may vary based on a number of factors, recent preliminary cost estimates from the U.S. Department of Defense for treatment in a certified opioid treatment program (OTP) provide a reasonable basis for comparison: methadone treatment, including medication and integrated psychosocial and medical support services (assumes daily visits): $\$ 126.00$ per week or $\$ 6,552.00$ per year; buprenorphine for a stable patient provided in a certified OTP, including medication and twice-weekly visits: $\$ 115.00$ per week or $\$ 5,980.00$ per year; naltrexone provided in an OTP, including drug, drug administration, and related services: $\$ 1,176.50$ per month or $\$ 14,112.00$ per year. 
significantly depending on the type of treatment, payer, and setting. Likewise, as far as we can tell, no study has directly compared the benefits produced by investing a similar amount of money in SCS with treatment for OUD, but the results could well depend on what assumptions are made about the extent to which SCSs change the behavior of PWUO outside the SCS facility.

In sum, although Kennedy, Karamouzian, and Kerr (2017) —who use the term supervised consumption facility for SCS - reported that "high-quality scientific evidence derived from the observational and simulation studies included in this review demonstrates the effectiveness of supervised consumption facilities in meeting their primary public health and order objectives" and "five [modeling] studies examined the impacts of Insite and found it to be cost-effective," when one examines these modeling studies carefully, they may be somewhat less definitive than the quotes suggest.

\section{Other Studies}

Our search also identified 18 studies that compared outcomes across nonsuitable comparison groups. An important example is comparing people who use SCSs frequently with those who use them infrequently or not at all, as in the Kerr et al. (2005) study mentioned earlier. This contrast could introduce selection bias that cannot necessarily be addressed by controlling for observable factors. ${ }^{38}$ Another 16 studies were cross-sectional, precluding any causal inferences, or evaluated outcomes without any control case. The remainder of the 65 individual studies identified were descriptive or qualitative in nature and described in the appendix of Pardo, Caulkins, and Kilmer (2018).

Finally, there are some outcomes, both positive and negative, that are potentially associated with SCSs but are difficult to evaluate. For example, if SCS implementation makes PWUO feel more respected and less stigmatized (as suggested in some of the qualitative literature; see, for example, Kerr et al., 2007; Krusi et al., 2009; Small et al., 2009; Kappel et al., 2016), it would be hard to capture this in a traditional quasi-experimental analysis. This is because (1) quantification of these outcomes is subjective and can be difficult to measure and (2) the effects could spill over to PWUO who do not use SCSs, as well as those in other neighborhoods, thus "contaminating" potential control groups and making it harder to identify an effect. Although the reduction in stigma might manifest itself in increased uptake of health and social services, there is also a dignity aspect to these types of interventions that is hard to measure but nonetheless important to consider. On the potential negative side, SCSs might—at least in theory - extend drug-using careers or reduce property values in their vicinity, but such outcomes were not considered by the quasi-experimental evaluations we reviewed.

\footnotetext{
${ }^{38}$ For example, drug users who are more risk averse or who are at a more stable point in their drug-using careers may both use the SCS more frequently and also avoid risky practices when using outside the SCS, but the less risky practices may be caused by the risk aversion or stability, not by the SCS, and might not be adequately accounted for by the control variables.
} 


\section{Legal Status in the United States}

We anticipate a situation in which cities and possibly states wish to proceed with SCSs before federal law is changed to allow them. This section reviews some relevant legal considerations for that situation, as well as associated issues, such as whether SCS staff could lose their medical practice licenses or face tort liability for harms caused by users whose consumption they supervise. In many cases, decisive statements are not possible because courts have not yet ruled on these matters.

\section{Federal Law}

The general consensus is that SCSs are proscribed under federal law; the fundamental question is whether the federal law definitively preempts state law in this area. Two sections of the CSA are the most relevant to the proscription of an SCS under federal law; one prohibits drug possession, and the other prohibits the use of property for manufacturing, distributing, or using a controlled substance. ${ }^{39}$ The CSA additionally explicitly states that the Commerce Clause applies to drug control issues in individual states because

[c]ontrolled substances manufactured and distributed intrastate cannot be differentiated from controlled substances manufactured and distributed interstate. Thus, it is not feasible to distinguish, in terms of controls, between controlled substances manufactured and distributed interstate and controlled substances manufactured and distributed intrastate. (21 U.S.C. 801[5]; emphasis added)

The application of the CSA to intrastate activity was challenged in Gonzales v. Raich. In Raich, one concern was that individuals growing cannabis (even for personal use) could affect the market as a whole, but it is unlikely that an SCS would have an impact on the supply and demand sides of the opioid market (Burris et al., 2009). However, other language in Raich suggests a broader concern: that allowing intrastate cannabis "would undermine the orderly enforcement of the entire regulatory scheme." This argument can more easily be applied to SCSs; systematically allowing illegal activity to occur could be seen as undermining the general regulatory scheme of the CSA.

However, the supremacy of the CSA was also considered in Gonzales v. Oregon. In this case, the state passed the Oregon Death with Dignity Act (ODWDA), which exempted physicians from criminal or civil liability if they prescribed or dispensed a dose of lethal drug to a terminally ill patient (and complied with the safeguards in the ODWDA). Then-U.S. Attorney General John Ashcroft issued a rule declaring that this was not legitimate medical practice and that the actions were unlawful under the CSA (Ashcroft, 2001). The Court held that the ODWDA was exactly the kind of state regulation of medical practice envisioned under the CSA (21 U.S.C. 903). Ashcroft did not have the authority to issue this rule, because the CSA is a

\footnotetext{
3921 U.S.C. 844 prohibits drug possession, and 21 U.S.C. 856 (the "crack house" statute) deals with properties in which drug manufacture or use is occurring; 21 U.S.C. 846 makes it a crime to conspire to violate the CSA, and 18 U.S.C. 2 makes it a crime to aid in the commission of any offense against the United States.
} 
statute aimed at combating recreational drug use and trafficking, not regulating the practice of medicine in general; " $[\mathrm{t}] \mathrm{o}$ read prescriptions for assisted suicide as 'drug abuse' under the CSA is discordant with the phrase's consistent use throughout the Act, not to mention its ordinary meaning" (Gonzalez v. Oregon). It is arguable whether applying the CSA to a state SCS would be construed as the same type of federal overreach that the Court rejected in Gonzales v. Oregon.

Ultimately, federal suppression of a state SCS would pose a serious question about the extent of Congress's authority under the Commerce Clause and the CSA (Burris et al., 2009). The Oregon decision demonstrates the limits of how far the CSA can stretch, and the Raich decision holds that states cannot pass laws protecting their citizens from federal enforcement of the CSA. However, neither addressed the question of whether the state law at issue was preempted by the CSA.

The Supremacy Clause in Article VI of the U.S. Constitution states that "the laws of the United States ... shall be the supreme law of the land" (U.S. Const. art. VI, cl. 2), establishing that federal law preempts state law on a certain issue if the two cannot coexist. However, a preemption analysis first requires a court to assess the purpose of the federal legislation to determine whether Congress intended to preempt state law. The CSA was drafted "to control illicit trafficking and to regulate legitimate uses of psychotropic substances in this country" (21 U.S.C. 801a[1]) and authorizes federal control of drugs (both legal and illegal) under the Commerce Clause of the Constitution. The statute specifically disclaims the government's intent to preempt state law:

No provision of this subchapter shall be construed as indicating an intent on the part of Congress to occupy the field in which that provision operates, including criminal penalties, to the exclusion of any State law on the same subject matter which would otherwise be within the authority of the State, unless there is a positive conflict between that provision of this subchapter and that State law so that the two cannot consistently stand together. (21 U.S.C. 903)

Given this statutory language, there is no express preemption of state laws in this domain. Preemption, ${ }^{40}$ however, does not necessarily need to be explicit; implied preemption may be established through judicial interpretation. There are several types of implied preemption. The one most likely applicable to a state-authorized SCS is obstacle preemption, in which the state law is somehow an obstacle to the purposes of the federal scheme. Assessing whether a state law "'stands as an obstacle to the accomplishment and execution of the full purposes and objectives of Congress' is a matter of judgment, to be informed by examining the federal statute as a whole and identifying its purpose and intended effects" (Crosby v. National Foreign Trade Council, quoting Hines v. Davidowitz).

One can imagine arguments as to why laws authorizing SCSs are not inconsistent with the purposes and objectives of the CSA. For example, creating a space in which PWUO can interact

\footnotetext{
40 The preemption doctrine is the idea that a higher legal authority (here, the federal government) will displace or preempt the law of a lower legal authority (here, a state government).
} 
with the medical system and perhaps become more comfortable with seeking treatment could be argued to be in line with the CSA objective of "combating drug abuse."

There is also case law suggesting that the CSA need not preempt state statutes that decriminalize or even legalize possession of drugs. Several state courts have ruled that their medical cannabis statutes are not preempted by federal law, and the U.S. Supreme Court has denied to review those decisions in several of the cases. ${ }^{41}$ The cases that have found that state laws are preempted by federal law are those addressing laws that required affirmative steps (e.g., providing unemployment benefits for those fired for medical cannabis use) rather than legalization or decriminalization. ${ }^{42}$ Note though that no federal courts have yet addressed the preemption argument with regard to medical cannabis statutes or other drug laws.

Even if a court did find that the CSA preempted state law, the federal government still could not compel a state to enforce the CSA or otherwise "enact or administer a federal regulatory program" (Printz v. United States). Nor could a state be compelled to pass a law coextensive with the CSA, as this would be considered commandeering: 43 "No matter how powerful the federal interest involved, the Constitution simply does not give Congress the authority to require the States to regulate" (New York v. United States).

In an article about state cannabis laws, Erwin Chemerinsky and colleagues set forth one way a state could exploit the limits on commandeering to counteract preemption (Chemerinsky et al., 2015). First, a state could repeal all its drug laws, and the federal government could not require the state to reenact those laws or to enforce the federal laws without running afoul of anticommandeering. The state could then pass new laws criminalizing opioid possession for all those who do not have a card or other evidence of participating in an SCS. Passing such a law that, in fact, criminalizes opioid possession for most people would be difficult to deem an "obstacle" to the CSA.

In the absence of explicit authorization on the federal level, the 2009 Ogden Memorandum (Ogden, 2009) and 2013 Cole Memorandum (Cole, 2013) show that the federal government can

\footnotetext{
${ }^{41}$ See, for example, Ter Beek v. City of Wyoming (unanimous decision holding that the CSA does not preempt the Michigan Medical Marihuana Act); People v. Crouse (holding that the CSA does not preempt state cannabis provisions and ordering the return of seized medical cannabis to the patient); Town of Wakefield $v$. Coakley (rejecting conflict and obstacle preemption arguments against state medical cannabis provisions); San Diego County v. San Diego NORML (allowing patients to obtain medical cannabis identification cards because they do not positively conflict with the CSA; simultaneous compliance with both sets of laws is not impossible, and the identification card provisions do not pose significant impediment to the objectives of the CSA); and City of Garden Grove v. Superior Court (holding that a medical cannabis patient was entitled under state law to the return of seized cannabis and that this return was not precluded by federal preemption). But see Mikos, 2013 (discussing both cases that have found preemption and those that have not).

42 See, for example, Emerald Steel Fabricators, Inc. v. Bureau of Labor \& Indus.; and Washburn v. Columbia Forest Prods., Inc.; but see also Bolitho, 2017 (criticizing the Obama administration's derogation of their duty under the Take Care Clause).

${ }^{43}$ In this context, commandeering refers to the federal government forcing a state to take an action that it otherwise would not-here, enforcing federal law. Commandeering has been held to be unconstitutional under the Tenth Amendment ("The powers not delegated to the United States by the Constitution, nor prohibited by it to the States, are reserved to the States respectively, or to the people").
} 
chose to deprioritize enforcing federal drug law in situations where persons are complying with state law, although these policies can easily change (e.g., the rescinding of the Cole Memorandum in January 2018). Although the attorney general could issue a regulation under the CSA that created exceptions to criminal liability for proprietors, employees, and clients of SCSs (21 U.S.C. 871), this seems unlikely in the immediate future. ${ }^{44}$

Congress has another option that is intermediate between explicit authorization and merely deprioritizing enforcement against SCSs. The Rohrabacher-Blumenauer Amendment blocks the U.S. Department of Justice from using federal funds to prevent state implementation of medical cannabis laws. ${ }^{45}$ Congress could pass a similar statute forbidding federal funds from being used to prevent implementation of SCSs, although SCSs do not enjoy the same level of popular support as medical cannabis (McGinty et al., 2018; Quinnipiac University, 2018).

\section{State and Local Authority}

The baseline ability for states or municipalities to open an SCS falls under their broad "police powers," 46 which include regulating public health. On the state level, the strongest legal path to an SCS would be authorization by a state legislature or by a referendum in ballot-initiative states. This course of action would decrease the likelihood of the SCS facing nuisance lawsuits or other violations of state land-use law. It would also provide the strongest position for both avoiding a challenge from the federal government and facing that challenge if it is brought to court; a similar situation currently exists in states that have authorized medicinal or recreational cannabis.

Another state-level approach would be administrative authorization of an SCS. This could take the form of an executive order from the governor or a regulation issued by a health agency. Executive power, however, generally only extends to authorizing activities that do not conflict with existing law, and executive authority to alter controlled substances rules is generally narrow, so a dissenting state legislature could challenge such an action as exceeding the executive's authority. On the other hand, health agencies in all states have rule-making authority to protect public health, and, in some states, health commissioners have the legal authority to

\footnotetext{
44 The U.S. attorney for Vermont has specifically denounced one county's intention to open an SCS (U.S. Department of Justice, U.S. Attorney's Office, District of Vermont, 2017), and in April 2018, a DEA spokesperson told the Washington Post that SCSs violate the CSA and are "subject to being prosecuted" (Cunningham, 2018). More recently, Deputy Attorney General Rod Rosenstein published an op-ed in the New York Times arguing that SCSs "create serious public safety risks," "destroy the surrounding community," "normalize drug use and facilitate addiction," and do not help PWUO to stop using (Rosenstein, 2018).

45 The current authorization of the amendment is included in the Consolidated Appropriations Act, 2018 (Pub. L. 115-141), passed on March 23, 2018.

${ }^{46}$ In constitutional law, the "police power" is a state's authority to regulate behavior within its borders for the betterment of its residents' health, safety, and general welfare. It is a loose, nebulous concept, which makes defining the boundaries and scope of the states' police powers a thorny legal question.
} 
allow activity related to controlled substances that would otherwise be prohibited under state law. ${ }^{47}$

A third approach would be authorization by a municipal governing body. ${ }^{48}$ Most cities have police powers within their borders analogous to the police powers of the state and therefore have wide discretion when it comes to implementing public health programs that are supported by reasonable evidence of efficacy. ${ }^{49}$ However, a local ordinance would be vulnerable to a challenge based on preemption by state drug laws. The SCS could also be vulnerable to interference by local police, as many municipal police departments are deputized to enforce state laws (Sherman, Hunter, and Rouhani, 2017). ${ }^{50}$

Before opening an SCS, a state or locality could seek a declaratory judgment in a federal court, ${ }^{51}$ which would lead to an official judicial interpretation of the applicability of the Controlled Substances Act to SCSs. This has the advantage of offering legal certainty, but if the court disagreed with the arguments above, it is possible that an SCS would be unable to open when it might have had the potential to operate indefinitely under legal uncertainty.

\section{Legal Issues for SCS Operators}

The biggest legal issue for operators of an SCS would be the "crack house statute," which states that it is criminally unlawful to

1. knowingly open, lease, rent, use, or maintain any place, whether permanently or temporarily, for the purpose of manufacturing, distributing, or using any controlled substance;

2. manage or control any place, whether permanently or temporarily, either as an owner, lessee, agent, employee, occupant, or mortgagee, and knowingly and intentionally rent, lease profit from, or make available for use, with or without compensation, the place for the purpose of unlawfully manufacturing, storing, distributing, or using a controlled substance. (21 U.S.C. 856)

\footnotetext{
${ }^{47}$ For example, New York public health law PBH Section 3381 does not itself decriminalize possession of hypodermic needles but rather allows the public health commissioner to authorize groups of individuals to possess the otherwise-illegal hypodermic syringes and needles under PEN Section 220.45 (see New York State Senate, undated). See also L.B. v. Town of Chester ("Once an individual is authorized by the Commissioner, that ends their liability as an 'unlawful' possessor under the Penal Law").

${ }^{48}$ See Wilson, 2018, for an argument for using cities' home-rule powers to create local strategies to combat drug addiction.

${ }^{49}$ This is the direction taken by some cities in establishing SEPs without authorization from their state governments (Burris et al., 1996).

${ }^{50}$ However, see Smith v. Hickenlooper (holding that law enforcement officers cannot claim a "crisis of conscience" as to whether to enforce the state versus federal law).

${ }^{51}$ A declaratory judgment provides a binding legal ruling resolving legal uncertainties for litigants in the early stages of a case or controversy, but it does not provide for enforcement of any remedies. It is generally requested when litigation seems likely or as part of a counterclaim to litigation that has already been filed.
} 
If found liable, property owners could be subject to criminal penalties, civil penalties, and civil forfeiture of the property on which the SCS was run (21 U.S.C. 856[b], 856[d]; 21 U.S.C. 881). However, conviction under the statute requires that the operation of the facility be "knowingly open[ed] . . . for the purpose of manufacturing, distributing, or using" a controlled substance (21 U.S.C. 856[a][1]). Prior case law has noted that purpose and knowledge are distinct elements. ${ }^{52}$ An SCS could argue that allowing drug use is not the purpose of the SCS, but the means to achieve other purposes (e.g., reduced overdose deaths, reduced blood-borne diseases), just as the purpose of using morphine in a hospital is not the use of morphine but the relief of pain. If the mere knowledge that drugs will be used on the premises is enough to establish that drug use is the purpose of the defendant, then the purpose element adds nothing to the state-of-mind requirement that is not already captured in the element of knowingly. ${ }^{53}$ Interpreting the statute without distinguishing the two terms violates the canon of construction of surplusage - that is, where one reading of a statute would make one or more parts of the statute redundant and another reading would avoid the redundancy, the second option is preferred. As an analogy, one might look to previous issues with SEPs. Drug paraphernalia laws generally prohibit the distribution of any item with knowledge that it will be used for the purpose of illegal drug consumption. In some states, local governments took the position that SEPs did not violate the paraphernalia laws in the first place because the purpose was not illegal drug consumption, but the betterment of public health. ${ }^{54}$ However, these cases generally involved conflicts between local and state law, rather than (hypothetical) state and federal law.

Other provisions of the CSA could also be used to counter an argument that an SCS violates Section $856 .{ }^{55}$ For instance, Section 1101 provides that

52 United States v. Chen.

${ }^{53}$ Look at the example of nuisance laws; in general, these laws prohibit activity that involves a property being used for unlawful conduct - e.g., N.Y. Penal Law Section 240.45 (New York State, 2008). If unauthorized by the state, an SCS would almost certainly be subject to this provision. However, if the SCS was state-authorized, the employees and clients would not be engaging in "unlawful conduct," nor would the premises be maintained for the purpose of engaging in unlawful conduct. Similarly, state authorization should give SCS operators a reasonable claim of "lawfulness" against a federal statute like Section 856 that is predicated on knowingly maintaining a place for the purpose of unlawful drug activity.

${ }^{54}$ See, for example, Spokane County Health District v. Brockett. This holds that

the needles at issue in this case are 'drug paraphernalia.' Those distributing the needles know they will be used to inject controlled substances unlawfully. Nevertheless, plaintiffs argue, the needle exchange program is authorized under the Washington Constitution, statutes granting broad powers to local health officials, and the omnibus AIDS act. Therefore, they conclude, the drug paraphernalia act, which is aimed at criminal conduct, simply does not apply to their actions. We agree.

${ }^{55}$ As this report was going to press, Kreit (forthcoming) published an article proposing a novel solution to the conflict between cities and the federal government over safe injection sites in the form of an obscure provision of the Controlled Substances Act that immunizes officials who are engaged in the enforcement of state and local laws relating to controlled substances. Although the CSA's immunity provision was likely written with undercover police officers in mind, the plain language of the law seems to apply to a government-run safe injection site. A handful of courts have already relied on the statute to immunize government officials who were engaged in 
Control of drug abuse requires the development of a comprehensive, coordinated long-term Federal strategy that encompasses both effective law enforcement against illegal drug traffic and effective health programs to rehabilitate victims of drug abuse. . . Local governments with high concentrations of drug abuse should be actively involved in the planning and coordination of efforts to combat drug abuse. (21 U.S.C. 1101; emphasis added)

It is possible to argue that opening an SCS in an area with high concentrations of drug use (a descriptor that applies to most of the areas that are considering opening a facility at the moment) is part of that area's plan to address drug use. Relatedly, Section 801a states that "nothing in the Convention will interfere with ethical medical practice in this country as determined by the Secretary of Health and Human Services on the basis of a consensus of the views of the American medical and scientific community" (21 U.S.C. 801a). The American Medical Association (2017) called for SCS pilot studies. Although the association does not dictate the policy of the U.S. Department of Health and Human Services, it is a strong signal from the medical community.

\section{Legal Issues for Medical Staff}

SCS medical staff commonly supervise and educate clients about safe and hygienic injection techniques but do not assist them with injection. This decreases their likelihood of liability; however, professional medical staff may rightly worry about losing their professional licensing or prescribing privileges. States could reassure doctors and nurses in this regard by introducing explicit statutory language making the SCS staff roles fully compliant with state law and endorsing their responsibilities in an SCS as good medical practice, not medical practice that poses a threat to public health, safety, or welfare (see, for example, Fitzgerald, Abel, and Bates, 2017). Medical staff might also worry about malpractice lawsuits because malpractice insurance generally excludes "criminal activity" from coverage, or courts might disallow insurance payouts in SCS cases as contrary to public policy.

Staff could have clients sign liability waivers. Although waivers would not protect doctors in instances of gross negligence or worse, having a signed waiver might deter lawsuits. Complicating that protection is the question of whether someone with OUD can give informed consent. It is generally clear that acute intoxication can lead to decisional impairment, and it is suggested that acute withdrawal may be similarly incapacitating (National Bioethics Advisory Commission, 1998, p. 9). The diagnosis of a substance use disorder does not indicate that decisionmaking capacity is necessarily impaired, but it is possible that there may be some

the enforcement of state medical marijuana laws. The logic of those decisions supports immunizing the operators of government-run safe injection sites. To be sure, there is other precedent that points toward a narrower reading of the immunity provision and so the case for applying it to safe injection sites is not open-and-shut. But if courts were to agree with the interpretation of the immunity provision outlined in this article, cities would be free to open safe injection sites without putting their employees at risk of federal prosecution.

We look forward to thinking more about this argument and seeing it discussed by legal scholars and those participating in SCS debates. 
impairment even outside the two acute circumstances described above. However, it seems unlikely that, in the case of an SCS, the argument that users were unable to give informed consent to a liability waiver because of a drug craving or a state of withdrawal would succeed. Because the SCS is not providing the drugs, there is no coercive inducement for users to sign the waiver. Clients are exchanging their ability to sue for the increased safety of sterile supplies, staff who can assist in case of an overdose, and perhaps even the opportunity to test their drugs. Anyone who did not want to sign a waiver at an SCS would be free to leave and to engage in the same actions they did prior to the opening of the SCS.

\section{Tort Liability}

If a client of an SCS leaves while under the influence and causes harm (e.g., falls asleep at the wheel on the way home), the client would certainly be individually liable for the damages. Would the SCS itself face any liability? Commercial providers of intoxicants have been liable under dram shop laws, but social hosts have been subject to a different legal framework (see, for example, Coppock, 2009). Under the common law, social hosts did not have a legal duty of care, but courts have more recently been inclined to find a legal duty. A case might be made that operators of SCSs should face the same type of liability as a social host at a "bring your own beer" party. ${ }^{56}$

\section{Insights from Interviews and Focus Groups}

Key informants were invited to comment on whether they thought that SCSs could help improve outcomes for PWUO and whether they believed that SCSs would be acceptable to the community ${ }^{57}$ Table 4.3 lists all themes that emerged and their frequency (i.e., low, medium, high, or very high frequency is indicated if the theme emerged in 1-25 percent, 26-50 percent, 51-75 percent, or 76-100 percent of all transcripts, respectively) that respond to these two questions. We include the indicator of the relative frequency with which the themes emerged to show how the emergence of themes differed between professionals and PWUO. Key findings pertaining to SCS based on insights from interviews and focus groups are discussed after the table.

\footnotetext{
56 The question of whether liability attaches to a social host is contingent on the five elements of a prima facie negligence case: the existence of a legal duty, breach of that duty, factual cause, proximate cause, and physical harm.

${ }^{57}$ The definition of SCSs that interviewers and focus group facilitators used to describe the intervention to key informants who were not familiar with SCSs is listed in Chapter 2 and in the interview and study protocols in Appendixes A-C of Ober et al. (2018).
} 
Table 4.3. SCS Themes Cited by Key Informants (Professionals and PWUO)

\begin{tabular}{|c|c|c|}
\hline \multirow[b]{2}{*}{ Theme } & \multicolumn{2}{|c|}{ Frequency } \\
\hline & $\begin{array}{l}\text { Professionals }^{\mathrm{a}} \\
\quad N=80\end{array}$ & $\begin{array}{l}\text { PWUO }^{\mathrm{b}} \\
N=79\end{array}$ \\
\hline \multicolumn{3}{|c|}{ Could SCS Help Improve Outcomes for PWUO? } \\
\hline \multicolumn{3}{|l|}{ Reasons SCS could help improve outcomes } \\
\hline SCSs could help prevent overdose deaths & Medium & Very high \\
\hline SCSs could help link PWUO to treatment and other resources & Medium & High \\
\hline SCSs could provide PWUO with a safe, nonjudgmental place to use & Low & Medium \\
\hline $\begin{array}{l}\text { SCSs could provide clean needles and information to prevent HIV, } \\
\text { hepatitis C, and abscesses }\end{array}$ & Low & Medium \\
\hline SCSs could provide drug composition testing & Low & Medium \\
\hline \multicolumn{3}{|l|}{ Reasons SCSs might not help improve outcomes } \\
\hline PWUO would view it as a law enforcement trap & Medium & High \\
\hline SCSs would be stigmatizing for PWUO & Medium & Medium \\
\hline $\begin{array}{l}\text { PWUO would be reluctant to travel to an SCS after purchasing drugs; } \\
\text { they want to use immediately }\end{array}$ & Low & High \\
\hline SCSs would enable or perpetuate opioid use & Medium & Very high \\
\hline SCSs would create a forum for drug dealers & Low & Medium \\
\hline PWUO do not want to be monitored while using & Low & Low \\
\hline SCSs increases risks for PWUO & Low & Low \\
\hline \multicolumn{3}{|l|}{ Not sure if SCSs would help improve outcomes } \\
\hline Need to see more evidence & Medium & Low \\
\hline \multicolumn{3}{|c|}{ Would SCS Be Acceptable to the Community? } \\
\hline $\begin{array}{l}\text { SCS implementation would be impeded by community values and local } \\
\text { culture }\end{array}$ & Very high & Medium \\
\hline $\begin{array}{l}\text { Community members would believe that an SCS enables or perpetuates } \\
\text { use }\end{array}$ & Medium & Low \\
\hline Community would say "not in my backyard" (NIMBY) & Medium & Low \\
\hline Stigma against PWUO would impede implementation & Medium & Medium \\
\hline An SCS is not a priority in the community right now & Medium & Low \\
\hline There might be more buy-in for SCSs in "less rural" areas & Low & N/A \\
\hline Rural communities do not have the resources to implement SCSs & Low & N/A \\
\hline Community members would believe that an SCS normalizes opioid use & Low & Low \\
\hline SCSs would affect neighborhoods and community resources & Low & Low \\
\hline $\begin{array}{l}\text { SCSs would "clean up" the streets and reduce strain on police and } \\
\text { emergency medical services }\end{array}$ & Low & Low \\
\hline
\end{tabular}

NOTES: N/A: Theme did not arise in any transcript; Low frequency: Theme arose in 1-25\% of transcripts; Medium frequency: Theme arose in $26-50 \%$ of transcripts; High frequency: Theme arose in $51-75 \%$ of transcripts; Very high frequency: Theme arose in $76-100 \%$ of transcripts.

a Professionals include all non-PWUO key informants who participated in an interview $(N=44)$ or focus group $(N=5$ groups and 36 providers) who were invited to participate based on their current profession. We are mindful that at least some of consulted PWUO may also be professionals. The use of professional in this report is not a comment on any individual's job status; rather, it is meant to capture the fact that we invited one group of key informants to inform the research project on the grounds of their occupations.

${ }^{b}$ PWUO who participated in ten focus groups.

Overall, there were four key insights about SCSs. First, key informants noted harm-reduction benefits to SCSs but also perceived drawbacks, such as that SCS may enable or perpetuate opioid use, and practical barriers, including PWUO's unwillingness to travel far to an SCS after purchasing opioids. Among the main benefits noted were preventing overdose and disease, offering links to treatment, providing a safe, judgment-free place for PWUO to use, and supporting drug composition testing. Some professionals and PWUO also thought that SCSs would enable or perpetuate opioid use. PWUO in all focus groups, rural and urban, saw an SCS as impractical because of the time needed to travel to an SCS after purchasing drugs, with rural 
PWUO emphasizing its impracticality because of overall transportation challenges. PWUO and professionals also worried that an SCS would be a law enforcement trap for PWUO. Some PWUO currently receiving MT noted that an SCS could provide a safe place to use while waiting to get into treatment.

Second, despite supporting the benefits of SCSs, interviewees and focus group participants generally believed that their communities as a whole likely would not currently accept an SCS as a viable strategy for helping address the opioid crisis because of cultural, resource, and practical barriers. Although this view was particularly salient among informants from the rural countiesneither of which currently has an SEP or adequate naloxone distribution, detoxification services, treatment provision, or sober living facilities - even urban informants who supported the concept felt that an SCS in their communities was not the current priority for addressing the opioid crisis. Key informants generally believed that putting funding into evidence-based programs that already have gained traction with community members would be a more prudent investment of limited available resources. Moreover, key informants cited multiple reasons that SCSs likely might not be acceptable to community members, including conservative political and cultural landscapes, lack of endorsement of a harm-reduction approach to OUD, long-standing stigma and fear around addiction, and general "NIMBYism" around placing such facilities in areas that would be accessible to PWUO.

Third, despite cultural and policy-related barriers, implementation may be more feasible in urban communities with existing (and perhaps more long-standing) harm-reduction programs, greater treatment resources, and adequate transportation, particularly if there is evidence to support it. Although some interviewees and focus group participants had reservations about SCSs and believed that their communities have other, more-pressing priorities, professional interviewees in the two urban communities were more inclined to believe that with education, evidence, and time, SCSs could eventually be accepted by community members, as SEP and MT have been accepted, at least by key stakeholders and policymakers. This was particularly the case in Cuyahoga County, where there has been an SEP since 1995, first authorized only through local emergency orders, and then legalized statewide in 2013.

Fourth, as with HAT, publication of evidence on SCSs and community education were seen as essential in fostering community acceptance of SCSs. A number of key informants expressed belief that more education around SCSs and published evidence would facilitate acceptance and generate potential community buy-in, on the assumption that there is adequate evidence of SCS effectiveness. This call for evidence was also made by key informants who were skeptical of SCSs or expressed reservations about the SCS model. Among other steps that could help address community concerns toward SCS were integrating the service with an existing medical facility and introducing a mobile supervision service, with the perceived dual benefit of reaching PWUO who might not come to a fixed SCS and of minimizing neighborhood concerns associated with a fixed SCS location. However, neither of these steps was fully endorsed as adequate for addressing community concerns. 


\section{Ideas for Informing Future Research and Policy Discussions}

\section{Conducting Clinical Trials of HAT and Hydromorphone in the United States}

Given (1) the increased mortality associated with fentanyl, (2) the fact that some people who use heroin may not respond well to existing medications for OUD, (3) HAT's successful implementation abroad, and (4) questions concerning whether the success would carry over to the United States, HAT clinical trials should be conducted in some of the U.S. jurisdictions that already provide a spectrum of social services and good accessibility to MTs for OUD. ${ }^{58}$ It seems less likely that one could get funding or political support for HAT research in places that were not already dedicating significant resources to MT and other services for PWUO. Conducting a multisite research study might also allow HAT to proceed in a limited fashion without requiring its schedule status to be changed and would provide evidence to inform such a rescheduling decision.

Few clinical studies have examined the effectiveness of treating OUD with hydromorphone (e.g., Dilaudid), but the early results are encouraging. Studies of hydromorphone would face fewer barriers in the United States than HAT studies would because hydromorphone is a Schedule II drug that can be prescribed for the treatment of pain. As noted in Chapter 3, the results of the first six-month phase of one Canadian RCT trial ( $N=202$ participants) suggest that supervised injectable heroin and supervised injectable hydromorphone showed similar effectiveness with regard to treatment retention, illicit heroin use, illegal activities, physical health, and mental health. Additionally, the hydromorphone group had significantly fewer serious adverse events related to the study medication than the HAT group.

Some U.S. jurisdictions and researchers may find injectable hydromorphone more attractive than HAT, given its Schedule II status (and likely greater community acceptability than heroin), while others may prefer to evaluate HAT given its much larger evidence base. Conducting trials with HAT and hydromorphone are not mutually exclusive options, and it may make sense to include them in the same study, as was done in Canada. Assessing the impact of injectable hydromorphone via clinical trials (with or without a HAT arm) would inform future regulatory decisions about using it as a medication treatment for OUD.

\section{Implications of the Nature of Evidence Concerning the Effects of SCSs}

There is debate about the level of proof policymakers should demand concerning the effectiveness of SCSs. One view is that SCSs should be subject to the same evidentiary standards

\footnotetext{
${ }^{58}$ In these studies, the injection of pharmaceutical-grade heroin would be supervised by medical officials in a designated health facility. In previous studies, the HAT participants were also given the opportunity to take home oral methadone to help prevent withdrawal.
} 
as any other health intervention. To simplify, that means RCTs are the gold standard and quasiexperiments can be informative, but associations (e.g., in cross-sectional analyses) are not taken as strong evidence of causality. Another view argues for lower standards of proof given (1) the urgency of the present crisis; (2) the fact that SCSs were created in response to health crises, not as part of research studies; (3) that it can be difficult to research controversial interventions focused on stigmatized populations in the United States, ${ }^{59}$ and (4) that the logic model underpinning the direct effects of SCSs is clear. When consumption leads to overdose in an SCS, prompt intervention is undertaken to manage the patient; indeed, there are no known overdose deaths from consumption inside an SCS (Hedrich, 2004; Potier et al., 2014). Likewise, injections within the SCS are done with sterile equipment, effectively eliminating the spread of bloodborne diseases and other harms from unhygienic injection from those supervised use sessions. (Many SCS clients also continue to inject drugs outside the SCS; see, for example, Kimber et al., 2003.)

Some may argue that it is unethical, indeed, even deadly, for policymakers to delay implementing SCSs. Others may want to wait for additional research for at least three reasons. First, SCSs (and HAT) can be controversial, so some policymakers will demand stronger forms of evidence before they act. Second, SCSs can require significant resources, and resources are limited, so the question is not just "do SCSs work?" but "are they cost-effective compared with other interventions intended to reduce overdose deaths and disease?" Third, and related, some of the claims concerning SCS effectiveness pertain to spillover effects on users' behavior outside the SCS for which the logic model is less direct.

With respect to overdose deaths, the claims in the literature are sometimes cautious and tend to focus on the absence of deaths within the facility. As noted, this does not tell us about the net effect of opening and operating an SCS. It is entirely plausible that SCSs also reduce overdose deaths outside the facility - for example, by educating clients about the risks of unsupervised or high-risk use or referring them to treatment. Thus, it is natural and appropriate to ask whether the total effect, indirect and direct, is large enough to show up in aggregate-level evaluations.

Unfortunately, we identified only two such studies with control groups examining fatal overdoses: one based on Vancouver from 2001 to 2005 and another focused on New South Wales from 1998 to 2006 . The former found a reduction in fatal overdoses associated with proximity to the SCS; the latter did not find any significant difference in fatal overdose rates between treatment and control areas.

With respect to the spread of HIV/AIDS, hepatitis, and the various other harms associated with sharing and reusing injection equipment, many reports are less cautious, already crediting SCSs with substantial effects beyond those stemming directly from supervision of consumption within the facilities' walls. For example, Pinkerton (2010) credits Insite with preventing 83.5 HIV infections per year, but most "were due to the syringe exchange program which would

\footnotetext{
${ }^{59}$ One of our reviewers offered an insightful reminder: "It is important to recall that the U.S. federal government for most of the past 30 years has prohibited funding to SEPs and prohibited funding of research on SEPs for much of this time as well. Developing evidence in support of these programs has been challenging as a consequence."
} 
prevent 80.7 infections even in the absence of any reduction in syringe borrowing rates as a consequence of safe injection within the supervised injection facility." That leaves just an estimated 2.8 per year (later increased to five to six per year by Pinkerton [2011]) to the supervision itself, which is more expensive than an SEP per person served and, in the eyes of stakeholders we interviewed in Ohio and New Hampshire, also generally more controversial.

Earlier research on SEPs included well-designed simulation and analytical models that estimated such indirect effects. Some evaluations of SCSs (e.g., Andresen and Boyd, 2010) draw on that prior work, but of the eight simulation studies reviewed by Kennedy, Karamouzian, and Kerr (2017), only one (Bayoumi and Zaric, 2008) explicitly sought to trace these indirect effects with a dynamic model customized to SCSs, and that study appears to have been flawed in other respects. More generally, Chapter 4 summarizes some of these existing SCS simulation studies, noting various limitations. We certainly hope more work will be done on that topic.

Our review suggests the evidence base is even weaker for understanding the effect of SCSs on treatment uptake — we did not identify any quasi-experimental studies measuring this outcome. Let us be clear: Absence of strong evidence from quasi-experimental studies or RCTs should not be conflated with evidence of absence of effects. More rigorous research is needed to quantify the total net effects of these programs in a way that allows fair comparison of them with other interventions. Especially given the magnitude of today's overdose crisis, public resources, especially scarce municipal funds, need to be allocated to the most cost-effective interventions.

For example, suppose a city receives a grant to spend $\$ 3$ million to address opioid problems, roughly the annual operating cost of Insite or MSIC. Its leaders may wonder how those dollars should be allocated to maximize the social benefit: Set up an SCS like MSIC or Insite? Launch several lower-cost overdose prevention sites that focus only on consumption supervision? Fund methadone treatment? Expand emergency medical services? Purchase and distribute more naloxone in the community? A combination of these activities? Something else? The same questions can be asked, albeit less precisely, in terms of political energy and capital. The existing literature cannot answer these questions. ${ }^{60}$

Scores of RCTs have been conducted on methadone, and our literature review identified ten RCTs for HAT. Given that an increasing number of jurisdictions are considering SCS pilots, one or more may want to consider launching that pilot in a way that supports an RCT or strong quasiexperimental evaluation. As with SEPs, discussions of using randomization to evaluate SCSs raise questions about whether this would be ethical. Although it is common in studies of medication to randomly assign participants to the promising drug or another medication (which may be a placebo), those who believe that SCSs reduce overdose deaths would likely argue that it is unethical to deny access to this intervention. There may be ways to address this problem, and we sketch a few possibilities here.

\footnotetext{
${ }^{60}$ This also raises the question of who will pay for this research. If the federal government is not supportive of SCS pilot programs, researchers will likely have to depend on state, philanthropic, or other funding sources.
} 
In places where resource constraints imply that an SCS will not be able to serve everyone, researchers may be able to leverage this scarcity. One approach, which can be controversial, is a wait-list study. In such a study design, those who express interest in using an SCS are randomly assigned to immediate access or to a wait list; those in the wait-list group will eventually receive SCS services, just not immediately. This approach has been used in the drug treatment literature and can measure the individual-level effects of an intervention (see, for example, Mattick et al., 2009; Magill and Ray, 2009).

A less controversial approach would be a multisite stepped-wedge cluster randomized trial, which is sometimes used to evaluate service delivery interventions (Hemming et al., 2015). ${ }^{61}$ With this approach, all sites will eventually get the treatment - in this case, an SCS — but the treatment is phased in over time. In some ways, this is similar to a wait-list design, but it is neighborhoods or communities, not individuals, that wait for a facility to be available. Even if randomization is not possible, much can be learned from rigorous quasi-experimental studies that compare neighborhood-level outcomes in places with and without an SCS.

Regardless of whether an RCT or quasi-experimental approach is employed, it would also be extremely useful to collect individual-level data on PWUO in the treatment and control regions before and after the SCS opens. One approach could use respondent-driven sampling to approximate a random sample of PWUO in each area (Heckathorn, 1997; Heckathorn and Cameron, 2017) and then follow them over time. ${ }^{62}$

Finally, we note that, although the research on SCSs is largely limited in its type and design, many SCSs have been around for 15 to 30 years and have survived multiple changes in local and national governments. Of course, persistence does not imply effectiveness; however, it seems unlikely that these programs - which were initially controversial in many places — would have such longevity if they had serious adverse consequences for their clients or for their communities. There are researchers and advocates who believe that during an emergency such as the present opioid crisis in the United States, the absence of a large downside risk for a program that has strong face validity may be sufficient for some policymakers to proceed, rather than

${ }^{61}$ According to Hemming et al. (2015),

The stepped wedge cluster randomised controlled trial is a relatively new study design that is increasing in popularity. It is an alternative to parallel cluster trial designs, which are commonly used for the evaluation of service delivery or policy interventions delivered at the level of the cluster. The design includes an initial period in which no clusters are exposed to the intervention. Subsequently, at regular intervals (the 'steps') one cluster (or a group of clusters) is randomised to cross from the control to the intervention under evaluation. This process continues until all clusters have crossed over to be exposed to the intervention. At the end of the study there will be a period when all clusters are exposed. Data collection continues throughout the study, so that each cluster contributes observations under both control and intervention observation periods. It is a pragmatic study design, giving great potential for robust scientific evaluations that might otherwise not be possible.

${ }^{62}$ A number of studies have used respondent-driven sampling to obtain information from PWUO (e.g., Paquette and De Wit, 2012; Ober et al., 2016). These individuals are typically compensated for their interviews and for recruiting other PWUO to join the study. 
waiting for further evidence. Nevertheless, if attempts to implement SCSs in the United States are successful, we hope a strong research component is incorporated into these efforts.

\section{Potential Limitations Concerning the Scale of These Interventions}

At present, no country has deployed HAT or SCSs at a large enough scale to reduce opioidrelated problems by a large proportion. By design, HAT is typically restricted to people who have tried conventional forms of treatment but are still injecting heroin and may not represent an attractive option for some eligible users. Perhaps surprisingly, interviewees noted that some HAT trials had difficulties recruiting a full complement of clients. That finding, however, may not hold in illicit drug markets overwhelmed by synthetic opioids. SCSs supervise only a very small proportion of all use sessions, even in cities such as Vancouver, where they are well established. Furthermore, the scale of opioid use in the United States is considerably larger than in other countries, particularly when one includes the nonmedical use of opioid analgesics that spans a wide range of social and geographical contexts.

Just to help put this in perspective: If we assume that there were on the order of 1.5 million DND heroin users in the United States in 2016 (based on methods employed by Kilmer et al., 2014) and that these individuals consumed an average of roughly twice a day (see Bayoumi and Zaric, 2008), that would equate to about 1.1 billion use sessions per year. ${ }^{63}$ As a reference, Vancouver's Insite SCS, which seems to be the largest in the world, supervised an average 415 injections per day in 2017 (Vancouver Coastal Health, 2018), or roughly 150,000 over the year. Even ignoring injection of methamphetamine and other drugs, or increases in injection frequency because of the diffusion of shorter-acting opioids like fentanyl, this suggests that the United States would need more than 7,000 SCSs like Insite to supervise those use sessions; as of 2017, there were only about 100 SCSs operating worldwide. ${ }^{64}$ Of course, this hypothetical about covering all injections tells us nothing about whether a local community should or should not adopt an SCS; it is only meant to help the reader think about scale and suggest that SCSs as implemented elsewhere would need to be part of a package of responses to the opioid crisis, not the only or primary piece.

The scope or reach of SCSs may also be limited by geography. In most of our focus groups with PWUO, participants felt that individuals would not travel far after they purchased their drugs to visit an SCS. That implies that any given SCS can cover only a fairly limited geographic footprint. Indeed, existing SCSs have relatively limited catchment areas; they are mostly used by clients who are already located in their general vicinity. Inasmuch as SCSs require some minimum scale to operate efficiently, this may limit SCSs to areas with a sufficient density of PWUO. There are also concerns with PWUO driving after taking opioids. When the opioid

\footnotetext{
63 This is an approximation that excludes injection by less frequent heroin users and those involving other drugs. It also does not account for the small share of DND heroin users who do not inject.

64 To put that into further perspective, in 2016 there were some 1,500 opioid treatment providers (Alderks, 2017) and more than 300 SEPs in the United States (amfAR, 2018).
} 
problem disproportionately involved heroin in urban areas, this might have mattered less, particularly in Europe, where public transit is often good. However, in the United States today, there are high rates of OUD in small towns and rural areas, not just cities.

Vancouver and other Canadian cities have taken an important step to expand the scale of SCSs by establishing a larger number of smaller overdose prevention sites throughout the city. These overdose prevention sites typically offer fewer services but focus on the core provision of supervision of injections, naloxone and oxygen administration, and calls to emergency medical services. Some overdose prevention sites are stand-alone tents, while others are co-located with other services for other populations, such as public housing facilities. They are run by nongovernmental organizations, some of which may receive partial support from the government. They tend to be cheaper than formal SCSs and are staffed by people trained in overdose prevention and response and not necessarily by licensed health care practitioners. They increase the capacity for the supervision of injections in two ways. First, they can relieve the pressure on existing SCSs that are at capacity; second, their geographical dispersion may expand the catchment area of existing services. Some jurisdictions have also implemented mobile SCSs in an effort to increase the geographical coverage of the service or, in some instances, lessen community opposition that a fixed location may invite. As we discuss below, this may be just one version of a general strategy of expanding supervision beyond specific, high-profile brickand-mortar sites.

With respect to HAT, even in those European countries where it is routinely offered, the uptake rates are low. In both the Netherlands and Switzerland circa 2009, approximately 5 percent of those with heroin use disorder participated in HAT (MacCoun and Reuter, 2011). Similar rates were reported to us by international interviewees from both countries. ${ }^{65}$ MacCoun and Reuter (2011) noted, "for reasons that scholars have hardly explored, this is not an attractive option to experienced addicts. There is more to heroin addiction than a craving for the drug." It is possible that more users in the United States would embrace HAT given the very high risk of overdose in a market saturated with synthetic opioids (Pardo and Reuter, 2018). Furthermore, even if the United States could get only 5 percent of 1.5 million DND heroin users into HAT, today that would equate to about 75,000 clients who may spend less in the illicit market, minimize their exposure to fentanyl, and possibly commit less crime or put themselves in dangerous situations to obtain drugs. Addressing a small proportion of an enormous problem can produce large benefits in absolute terms.

In this regard, it is useful to recall the contextual differences in which HAT has been operating. In the Netherlands and in Switzerland, the population of PWUO has been declining, with relatively few new PWUO entering the treatment system. For that reason, there is relatively little pressure to expand HAT. By contrast, British Columbia and, increasingly, other Canadian provinces are dealing with large PWUO populations and an influx of fentanyl, not dissimilar to

\footnotetext{
${ }^{65}$ However, note that in these countries, HAT programs are restricted to people with previous unsuccessful treatment episodes. For that reason, the HAT-eligible population is smaller than the total population of opioid users.
} 
some regions in the United States. The increasing availability of fentanyl suggests that we should consider interventions like HAT to reduce PWUO exposure to extremely dangerous drugs. In such a context, the issue of HAT scalability is likely to be more pronounced, and it is therefore not surprising that Canada is at the forefront of innovative efforts to expand the provision of HAT and other overdose-mitigation services. British Columbia has opened a small number of injectable opioid programs providing hydromorphone, with the possibility of future expansion to heroin. Other provinces, including Alberta and Ontario, are considering or planning similar programs. ${ }^{66}$ British Columbia is also implementing a pilot of a pharmacy-based injectable program, which would offer injections supervised by trained pharmacists and would serve communities where more-comprehensive models of care may not be feasible.

\section{Are HAT and SCS Archetypes of Broader Collectives of Strategies?}

The previous section is rather pessimistic. If either HAT or SCS programs were to cover a large share of the opioid problem in the United States, they would have to be deployed at a much greater scale than has been achieved elsewhere. However, it might be constructive to view HAT and particularly SCSs as exemplars of a broader strategy, not as the only option within their class.

Until the beginning of the 21st century in the United States, MT for OUD largely meant methadone and nothing else. Part of the innovation of HAT might have been offering heroin in particular; however, part might have been the fact that it was injected, part might have been that consumption was supervised, and part might have simply been that clients had a choice of medications. It is common for there to be a variety of medicines that can help with one particular disease or condition, and different people do better or worse with one versus another. Viewed that way, HAT's success might be seen not just as a call for treating OUD with heroin specifically; it is also a call for considering broadening doctors' armamentarium for treating OUD more generally. Of course, the specific RCT results for HAT apply only to heroin. But if a jurisdiction found HAT appealing but could not pursue it for legal, political, or bureaucratic reasons, it might respond by investigating other avenues, such as supervised injectable hydromorphone, not by giving up altogether on expanding the options for MT.

Similarly, it is worth asking whether the success of SCSs depends on the first or the second $s$ in the abbreviation. That is, is the key the supervision of the use or the fact that it happens at a site or facility? Some SCS models require significant upstart and operating costs, with Vancouver's Insite costing on average about $\$ 7.50$ per consumption episode and so perhaps $\$ 15$ per day of supervised use. Furthermore, brick-and-mortar buildings explicitly dedicated to supervising drug consumption may face NIMBY problems even if the public is supportive of SCSs overall.

\footnotetext{
${ }^{66}$ A small injectable hydromorphone program is already operating in Ottawa ("Residential Opioid Program Giving Drug Users Chance at New Life," 2018).
} 
But perhaps what is essential is the service (namely, someone supervising the use session) rather than the site - that is, the physical circumstances of how the service is provided. If so, there may be other ways of offering supervision that are cheaper and still responsive to PWUO needs. To start with, the training necessary to carry and administer naloxone, and to call 911, is not extensive; it might be something that trained laypeople, not just nurses and other health care professionals, could provide. This is broadly the direction taken by overdose prevention sites in British Columbia, which work to expand supervision services at lower costs. Another option would be to co-locate supervision services within an existing facility serving PWUO, such as an existing SEP. This might lower costs of supervision and be more acceptable to the community if the community is already accustomed to service provision to PWUO in that facility. Again, colocation with existing services, although not necessarily services for PWUO only, is the model taken in some new SCSs in Canada.

A small number of locations in Canada are forgoing a fixed physical building altogether and offering a mobile service via a supervision van. As discussed in Strang and Taylor (2018), this model potentially offers interesting benefits, such as the ability to reach out to PWUO unable to travel to a fixed location, but also suffers from notable drawbacks, such as limited capacity or the need of users to conform to the site's schedule if it travels around multiple locations a day.

Perhaps supervision could be delivered without even a van if social workers or other authorized supervisors visited clients in their homes or other prearranged premises, either according to a scheduled timetable or as an on-demand service. ${ }^{67}$ There would clearly be challenges, such as how to document the provision of services (e.g., for the purposes of reimbursement) or how to avoid putting any participants in legal danger. Nevertheless, it might be worth asking whether some such approach might get a larger proportion of drug use sessions supervised more quickly than expansion of traditional SCS can manage. Breaking out of the brick-and-mortar SCS paradigm might be particularly relevant in rural areas, where there may not be enough PWUO living within the short travel radius of an SCS that is dictated by PWUO's unwillingness to travel far after purchasing drugs and before using them. ${ }^{68}$

None of these questions has answers at present, but the horrific death toll wrought by opioids should drive true innovation, not just extension of best practice. After all, the old adage says that necessity is the mother of invention, not just replication of existing evidence-based practice.

\footnotetext{
${ }^{67}$ One could even imagine a ride-hailing-style smartphone app that connects trained drug use observers to people who want to use opioids, but with the health department, not the user, compensating the observers for their service. ${ }^{68}$ Another alternative might be a well-funded public relations campaign highlighting the dangers of using opioids alone. If "friends don't let friends drive drunk," then perhaps also "friends should not let friends inject alone." Many places have "don't use alone" campaigns, but this messaging could target more than PWUO. If a city spent $\$ 3$ million per year on such a public education campaign, would that get more or fewer opioid use sessions observed than if the same money were invested in SCSs located in physical buildings? And how would one factor in that the supervision resulting from a public education campaign may be qualitatively different from that where a medically trained authorized supervisor is present?
} 


\section{Appendix. A Brief History of Heroin-Assisted Treatment Discussions in the United States}

HAT is a recurring topic for discussion in U.S. drug policy but usually under the more challenging name heroin maintenance. There was some high-level interest in the 1950s, a very prominent debate over the possibility of a trial in New York City in the 1970s, and then a brief resurgence of interest in the late 1990s. It is instructive to examine each of these episodes.

In the 1950s, when heroin use was a minor problem (and seen as such), elite groups were willing to consider radical change to what had become an extremely punitive regime; Congress in 1951 had raised the maximum penalty for first narcotics offense to five years. The American Bar Association joined with the American Medical Association in 1955 to form the Committee on Narcotic Drugs, which issued two reports (Joint Committee of the American Bar Association and the American Medical Association on Narcotic Drugs, 1961). Although the term heroin maintenance was not used, that was in fact the implication of the program that the committee's interim report suggested should be tried on an experimental basis (Musto, 1999). In this respect, it was resuscitating the "narcotics clinics" that had operated in a few cities in the early postHarrison era before Supreme Court rulings closed them. The committee made extensive reference to the British experience, permitting doctors to maintain addicts on heroin. The Federal Bureau of Narcotics, the predecessor to DEA, attacked the American Bar Association and American Medical Association report aggressively. Perhaps as a consequence, the report gained no policy traction, despite its sponsorship by two very prestigious professional associations.

The most serious effort at offering heroin to those already addicted was a proposal for a trial of heroin maintenance in New York City in the early 1970s. Prominent New York politicians (e.g., mayoral candidates) had been including at least rhetorical references to maintenance as a possible option since the beginning of the heroin epidemic in the mid-1960s (Bayer, 1976). Although methadone clinics were operating throughout the city, many dependent heroin users were unwilling to enter treatment.

The Vera Institute, a new but already well-respected social policy research organization, proposed in 1970 to conduct a modestly scaled heroin maintenance experiment: just 300 male participants ages 21-25 who had been addicted for at least two years and been in and out of treatment:

These addicts were to be maintained on heroin injected on clinic premises by medical personnel for a period of no longer than 6 months, during which time the full range of social and medical services would be brought to play in an effort to prepare the 'patient' for transfer to more conventional treatment. Such treatment would range from methadone maintenance on the Dole-Nyswander model to drug-free therapeutic communities. (Bayer, 1976) 
Again, the perceived success of the British system was a major factor in persuading the Vera staff to offer this proposal (Judson, 1974). Vera planned to adopt the British system of providing the smallest amount of heroin that the patient would accept but planned to provide many more supplemental services than were available in the United Kingdom.

New York City Mayor John Lindsay was cautious about this proposal, as were his staff. The final proposal for the trial in 1972 involved only 150 male addicts, and eligibility criteria were strengthened. Still, the public response was almost universally negative from many and varied sources. Charles Rangel, a congressman representing Harlem, cautioned, "[We must] recognize heroin for what it is - a killer, not a drug on which a human should be maintained" (Rangel, 1972). Vincent Dole, the scientist principally responsible for the discovery of methadone treatment, wrote an editorial in the Journal of the American Medical Association attacking the proposal on a variety of grounds (Dole, 1972). The New York Times published many articles highlighting the negative consequences of the drug (see Reuter and MacCoun, 2002). Even though it was designed as a transitional treatment, the opposition characterized it as involving lifetime addiction to heroin. The proposal had many prominent enemies. The support of a variety of criminal justice officials, including Police Commissioner Patrick Murphy and a number of the elected district attorneys, was insufficient. It simply faded away by 1975.

The Swiss HAT trials did not generate a specific proposal for HAT in the United States, but they did generate a hearing designed to show their failure, with a highly selective choice of hostile witnesses to appear before the National Security, International Affairs and Criminal Justice Subcommittee of the House Government Reform and Oversight Committee (MacCoun and Reuter, 2001). In 1998, David Vlahov, an influential Johns Hopkins University academic, proposed a heroin maintenance trial, partly in response to the AIDS epidemic. The negative political reaction was instantaneous, from the governor on down. Even Kurt Schmoke, the Baltimore mayor who had previously endorsed major drug policy reforms, opposed it and censured his health commissioner for endorsing the proposal (MacCoun and Reuter, 2001). Vlahov's work contributed to the formation of NAOMI, which in turn led to the Vancouver experiment discussed throughout the report. However, there was again no traction in the United States (Pearson, 2004). 


\section{References}

Ahamad, K., T. Kerr, S. Nolan, and E. Wood, Moving Towards Improved Access for EvidenceBased Opioid Addiction Care in British Columbia: British Columbia Centre for Excellence in HIV/AIDS, Vancouver: British Columbia Centre for Excellence in HIV/AIDS, May 2016. As of September 26, 2018:

http://www.cfenet.ubc.ca/sites/default/files/uploads/news/releases/improved-access-opioidaddiction-care-bc-final-jun1.pdf

Alderks, Cathie E., "Trends in the Use of Methadone, Buprenorphine, and Extended-Release Naltrexone at Substance Abuse Treatment Facilities: 2003-2015 (Update)," CBHSQ Report, Substance Abuse and Mental Health Services Administration, August 22, 2017. As of November 3, 2018: https://www.samhsa.gov/data/sites/default/files/report_3192/ShortReport-3192.html

Ally, M. A., S. Brochu, and E. Blais, "Effet de l'implantation d'une clinique de prescription médicale d'héroïne sur l'environnement communautaire," Canadian Journal of Public Health, Vol. 102, No. 3, 2011, pp. 183-187.

Allyn, Bobby, "Cities Planning Supervised Drug Injection Sites Fear Justice Department Reaction," Morning Edition, National Public Radio, June 12, 2018. As of November 2, 2018 : https:/www.npr.org/sections/health-shots/2018/07/12/628136694/harm-reductionmovement-hits-obstacles

American Medical Association, "AMA Wants New Approaches to Combat Synthetic and Injectable Drugs,” press release, June 12, 2017. As of October 31, 2018:

https://www.ama-assn.org/ama-wants-new-approaches-combat-synthetic-and-injectabledrugs

amfAR, Opioid and Health Indicator Database, data pulled September 30, 2018. As of September 30, 2018:

http://opioid.amfar.org/

Andresen, Martin A., and Neil Boyd, "A Cost-Benefit and Cost-Effectiveness Analysis of Vancouver's Supervised Injection Facility," International Journal of Drug Policy, Vol. 21, No. 1, 2010, pp. 70-76.

Andresen, Martin A., and Ehsan Jozaghi, "The Point of Diminishing Returns: An Examination of Expanding Vancouver's Insite," Urban Studies, Vol. 49, No. 16, 2012, pp. 3531-3544.

Ashcroft, John, "Dispensing of Controlled Substances to Assist Suicide," memorandum to Asa Hutchinson, Washington, D.C.: Office of the Attorney General, November 6, 2001. As of 
November 12, 2018:

https://www.law.cornell.edu/primary_sources/ashcroft_directive.html

Associated Press-NORC Center for Public Affairs, Americans Recognize the Growing Problem of Opioid Addiction, Chicago, 2018. As of November 1, 2018:

http://www.apnorc.org/projects/Pages/HTML\%20Reports/Americans-Recognize-theGrowing-Problem-of-Opioid-Addiction.aspx

Bardwell, G., A. Scheim, S. Mitra, and T. Kerr, "Assessing Support for Supervised Injection Services Among Community Stakeholders in London, Canada," International Journal of Drug Policy, Vol. 48, 2017, pp. 27-33.

Barry, Colleen L., Susan G. Sherman, and Emma E. McGinty, "Language Matters in Combatting the Opioid Epidemic: Safe Consumption Sites Versus Overdose Prevention Sites," American Journal of Public Health, Vol. 108, No. 9, September 2018.

Bayer, Ronald, "Heroin Maintenance, the Vera Proposal and Narcotics Reform: An Analysis of the Debate, 1971-1973," Contemporary Drug Problems, Vol. 4, No. 3, 1976, pp. 297-322.

Bayoumi, A. M., C. Strike, J. Jairam, T. Watson, E. Enns, G. Kolla, A. Lee, S. Shepherd, S. Hopkins, M. Millson, L. Leonard, G. Zaric, J. Luce, N. Degani, B. Fischer, R. Glazier, P. O'Campo, C. Smith, R. Penn, and M. Brandeau, Report of the Toronto and Ottawa Supervised Consumption Assessment Study, Toronto: St Michael's Hospital and the Dalla Lana School of Public Health, University of Toronto, 2012.

Bayoumi, Ahmed M., and Gregory S. Zaric, “The Cost-Effectiveness of Vancouver's Supervised Injection Facility," Canadian Medical Association Journal, Vol. 179, No. 11, 2008, pp. 1143-1151.

Beletsky, Leo, Corey S. Davis, Evan Anderson, and Scott Burris, "The Law (and Politics) of Safe Injection Facilities in the United States," American Journal of Public Health, Vol. 98, No. 2, 2008, pp. 231-237.

Blanken, Peter, Vincent M. Hendriks, Jan M. Van Ree, and Wim van den Brink, "Outcome of Long-Term Heroin-Assisted Treatment Offered to Chronic, Treatment-Resistant Heroin Addicts in the Netherlands," Addiction, Vol. 105, No. 2, 2010, pp. 300-308.

Bolitho, Zachary C., "The U.S. Constitution, the U.S. Department of Justice, and State Efforts to Legalize Marijuana," Lincoln Memorial University Law Review, Vol. 4, No. 42, 2017, pp. $42-85$.

Boyd, S., D. Murray, and D. MacPherson, "Telling Our Stories: Heroin-Assisted Treatment and SNAP Activism in the Downtown Eastside of Vancouver," Harm Reduction Journal, Vol. 14, No. 1, 2017. 
British Columbia Centre on Substance Use, Guidance for Injectable Opioid Agonist Treatment for Opioid Use Disorder, Vancouver, 2017. As of November 1, 2018:

http://www.bccsu.ca/wp-content/uploads/2017/10/BC-iOAT-Guidelines-10.2017.pdf

British Columbia Ministry of Health, Office of the Provincial Health Officer, BC Opioid Substitution Treatment System: Performance Measures 2014/2015-2015/2016, Vancouver, March 2017. As of November 1, 2018:

https://www2.gov.bc.ca/assets/gov/health/about-bc-s-health-care-system/office-of-theprovincial-health-officer/reports-publications/special-reports/bc-ost-system-measures-14-15and-15-16.pdf

Brophy, Daragh, "We Have a Location for the First Supervised Drug Injecting Room-What Happens Next?" The Journal, March 10, 2018. As of November 26, 2018 : https://www.thejournal.ie/supervised-injecting-dublin-what-happens-now-3894154Mar2018/

Brown, Jennifer, and Jon Murray, "Denver Heroin Users Could Use Supervised Injection Site If Proposal Passes Multiple Hurdles," Denver Post, November 6, 2017. As of November 26, 2018:

https://www.denverpost.com/2017/11/05/denver-heroin-drug-users-supervised-injection-siteproposal/

Burris, Scott, Evan D. Anderson, Leo Beletsky, and Corey S. Davis, "Federalism, Policy Learning, and Local Innovation in Public Health: The Case of the Supervised Injection Facility," St. Louis University Law Journal, Vol. 53, 2009.

Burris, S., D. Finucane, H. Gallagher, and J. Grace, "The Legal Strategies Used in Operating Syringe Exchange Programs in the United States," American Journal of Public Health, Vol. 86, No. 8, 1996, pp. 1161-1166.

Cameron, Elizabeth, "Why Alberta Plans to Prescription Opioid Injections,” Star Calgary, May 1, 2018. As of November 1, 2018:

https://www.thestar.com/calgary/2018/05/01/why-alberta-plans-to-offer-prescription-opioidinjections.html

Case, Anne, and Angus Deaton, "Rising Morbidity and Mortality in Midlife Among White NonHispanic Americans in the 21st Century," Proceedings of the National Academy of Sciences, Vol. 112, No. 49, 2015, pp. 15078-15083.

Caulkins, Jonathan P., and Carolyn Coulson, "To Schedule or Not to Schedule: How Well Do We Decide?” Journal of Global Drug Policy and Practice, Vol. 5, No. 4, 2011.

Caulkins, Jonathan P., Beau Kilmer, Peter H. Reuter, and Greg Midgette, “Cocaine's Fall and Marijuana's Rise: Questions and Insights Based on New Estimates of Consumption and Expenditures in U.S. Drug Markets," Addiction, Vol. 110, No. 5, 2015, pp. 728-736. 
CBC News, "Vancouver's Heroin Users Get Safe-Injection Site," September 15, 2003. As of November 12, 2018:

https://www.cbc.ca/news/canada/vancouver-s-heroin-users-get-safe-injection-site-1.361046

$\mathrm{CDC}-\mathrm{See}$ Centers for Disease Control and Prevention.

Centers for Disease Control and Prevention, "HIV Surveillance: United States, 1981-2008," Morbidity and Mortality Weekly Report, Vol. 60, No. 21, 2011, pp. 689-693.

—_ "Laws Related to Syringe Exchange," webpage, last updated September 28, 2017a. As of November 1, 2018:

https://www.cdc.gov/hepatitis/policy/SyringeExchange.htm

- CDC WONDER, database, data pulled November 2017b. As of September 26, 2018 :

https://wonder.cdc.gov/

__ , "Drug Overdose Deaths in the United States, 1999-2017," Data Brief 329, 2018a. As of November 29, 2018:

https://www.cdc.gov/nchs/data/databriefs/db329_tables-508.pdf\#4

—_, "CDC Director's Media Statement on U.S. Life Expectancy," November 29, 2018b. As of November 29, 2018:

https://www.cdc.gov/media/releases/2018/s1129-US-life-expectancy.html

Center for Substance Abuse Treatment, Clinical Guidelines for the Use of Buprenorphine in the

Treatment of Opioid Addiction, Treatment Improvement Protocol Series 40, Rockville, Md.:

Substance Abuse and Mental Health Services Administration, 2004.

Chalfin, Aaron, "Economic Costs of Crime," Wiley Online Library, October 2015.

Chemerinsky, Erwin, Jolene Forman, Allen Hopper, and Sam Kamin, "Cooperative Federalism and Marijuana Regulation," UCLA Law Review, Vol. 61, No. 74, 2015, pp. 77-122.

Christie, Chris, Charlie Baker, Roy Cooper, Patrick J. Kennedy, and Pam Bondi, The President's Commission on Combating Drug Addiction and the Opioid Crisis, November 2017.

Ciccarone, D., J. Ondocsin, and S. G. Mars, "Heroin Uncertainties: Exploring Users' Perceptions of Fentanyl-Adulterated and -Substituted 'Heroin,"' International Journal of Drug Policy, Vol. 46, 2017, pp. 146-155.

City of Garden Grove v. Superior Court, 2008 Cal LEXIS 3517, California, 2008.

Clinical Guidelines on Drug Misuse and Dependence Update 2017 Independent Expert Working Group, "Drug Misuse and Dependence: UK Guidelines on Clinical Management," London: UK Department of Health, 2017.

Code of Federal Regulations, Title 21, Part 5.10, Delegations from the Secretary, the Assistant Secretary for Health, and Public Health Service Officials.

— Title 21, Part 1301.41, Hearings Generally. 
- Title 28, Part 0.100, General Functions.

Cole, James M., "Guidance Regarding Marijuana Enforcement," memorandum for selected U.S. attorneys, August 29, 2013.

Collins, Alexandra B., Ricky N. Bluthenthal, Jade Boyd, and Ryan McNeil, "Harnessing the Language of Overdose Prevention to Advance Evidence-Based Responses to the Opioid Crisis," International Journal of Drug Policy, Vol. 55, 2018, pp. 77-79.

Coppock, Lee A., "Social Host Immunity: A New Paradigm to Foster Responsibility," Capital University Law Review, Vol 38, No. 19, 2009, pp. 21-23.

Crosby v. National Foreign Trade Council, 530 U.S. 363, 2000.

CTV News, "Experts Table Findings on Drug-Injection Site," 2008. As of November 12, 2018 : https://www.ctvnews.ca/experts-table-findings-on-drug-injection-site-1.288775

Cunningham, Paige Winfield, "The Health 202: Supervised Injection Facilities Are Illegal in the United States. These Cities Want to Open Them Anyway," Washington Post, April 30, 2018.

Dasgupta, Nabarun, Leo Beletsky, and Daniel Ciccarone, "Opioid Crisis: No Easy Fix to Its Social and Economic Determinants," American Journal of Public Health, Vol. 108, No. 2, 2018, pp. 182-186.

DEA-See Drug Enforcement Administration.

Demaret, I., E. Quertemont, G. Litran, C. Magoga, C. Deblire, N. Dubois, J. de Roubaix, C. Charlier, A. Lemaître, and M. Ansseau, "Efficacy of Heroin-Assisted Treatment in Belgium: A Randomised Controlled Trial," European Addiction Research, Vol. 21, No. 4, 2015, pp. 179-187.

Des Jarlais, Don C., Kamyar Arasteh, and Holly Hagan, "Evaluating Vancouver's Supervised Injection Facility: Data and Dollars, Symbols and Ethics," CMAJ, Vol. 179, No. 11, 2008, pp. 1105-1106.

Dick, Andrew W., Rosalie L. Pacula, Adam J. Gordon, Mark Sorbero, Rachel M. Burns, Douglas Leslie, and Bradley D. Stein, "Growth in Buprenorphine Waivers for Physicians Increased Potential Access to Opioid Agonist Treatment, 2002-11," Health Affairs, Vol. 34, No. 6, 2015, pp. 1028-1034.

Dole, V. P., "Comments on Heroin Maintenance,” JAMA, Vol. 220, No. 11, 1972, pp. 14931493.

Donnelly, Neil, and Nicole Mahoney, Trends in Property and Illicit Drug Crime Around the Medically Supervised Injecting Centre in Kings Cross: 2012 Update, Sydney: NSW Bureau of Crime Statistics and Research, July 2013. 
Dowell, D., E. Arias, K. Kochanek, R. Anderson, G. P. Guy, J. L. Losby, and G. Baldwin, "Contribution of Opioid-Involved Poisoning to the Change in Life Expectancy in the United States, 2000-2015," JAMA, Vol. 318, No. 11, 2017, pp. 1065-1067.

Drug Enforcement Administration, “Application for Registration Under Controlled Substances Act of 1970," webpage, undated. As of November 1, 2018:

https://apps.deadiversion.usdoj.gov/webforms/jsp/regapps/common/newAppLogin.jsp

—, 2017 National Drug Threat Assessment, Washington, D.C., October 2017. As of November 1, 2018:

https://www.dea.gov/sites/default/files/2018-07/DIR-040-17_2017-NDTA.pdf

Emerald Steel Fabricators, Inc. v. Bureau of Labor and Indus., 230 P.2d. 518, Oregon, 2010.

Espelt, A., J. R. Villalbi, M. Bosque-Prous, O. Pares-Badell, M. Mari-Dell'Olmo, and M. T. Brugal, "The Impact of Harm Reduction Programs and Police Interventions on the Number of Syringes Collected from Public Spaces: A Time Series Analysis in Barcelona, 20042014," International Journal of Drug Policy, Vol. 50, 2017, pp. 11-18.

European Centre for Disease Control and Prevention and European Monitoring Centre for Drug Addiction, Prevention and Control of Infectious Diseases Among People Who Inject Drugs, Stockholm, 2011. As of November 1, 2018:

https://ecdc.europa.eu/sites/portal/files/media/en/publications/Publications/111012_Guidance _ECDC-EMCDDA.pdf

European Monitoring Centre for Drugs and Drug Addiction, New Heroin-Assisted Treatment, Lisbon, Portugal, April 2012.

- Belgium Country Drug Report 2017, Luxembourg: Publications Office of the European Union, 2017a. As of August 5, 2018:

https://eurotox.org/wp/wp-content/uploads/Belgium_Country-Drug-Report-2017.pdf

- Health and Social Responses to Drug Problems: A European Guide, Luxembourg: Publications Office of the European Union, $2017 \mathrm{~b}$.

- Drug Consumption Rooms: An Overview of Provision and Evidence, Lisbon, Portugal, June 2018a.

- Netherlands: Country Drug Report 2018, Lisbon, Portugal, 2018b. As of August 6, 2018:

http://www.emcdda.europa.eu/countries/drug-reports/2018/netherlands/harm-reduction_en

- United Kingdom: Country Drug Report 2018, Lisbon, Portugal, 2018c. As of September 27, 2018:

http://www.emcdda.europa.eu/countries/drug-reports/2018/united-kingdom_en

FDA—See U.S. Food and Drug Administration. 
Federal Office of Public Health, "Substitution-Assisted Treatment with Diacetylmorphine (Heroin)," 2018. As of November 12, 2018:

https://www.bag.admin.ch/bag/de/home/gesund-leben/sucht-und-gesundheit/suchtberatungtherapie/substitutionsgestuetzte-behandlung/heroingestuetzte-behandlung.html

Fernandes, Ricardo M., Maria Cary, Gonçalo Duarte, Gonçalo Jesus, Joana Alarcão, Carla Torre, Suzete Costa, João Costa, and António Vaz Carneiro, "Effectiveness of Needle and Syringe Programmes in People Who Inject Drugs: An Overview of Systematic Reviews," BMC Public Health, Vol. 17, No. 1, 2017, p. 309.

Fernandez, Humberto, and Therissa A. Libby, Heroin: Its History, Pharmacology and Treatment, 2nd ed., Center City, Minn.: Hazelden, 2010.

Fischer, B., and C. Allard, Feasibility Study on "Supervised Drug Consumption" Options in the City of Victoria, Vancouver: Centre for Addictions Research of British Columbia, 2007.

Fischer, Benedikt, Eugenia Oviedo-Joekes, Peter Blanken, Christian Haasen, Jürgen Rehm, Martin T. Schechter, John Strang, and Wim van den Brink, "Heroin-Assisted Treatment (HAT) a Decade Later: A Brief Update on Science and Politics," Journal of Urban Health, Vol. 84, No. 4, 2007, pp. 552-562.

Fitzgerald, Jacqueline, Melissa Burgess, and Lucy Snowball, Trends in Property and Illicit Drug Crime Around the Medically Supervised Injecting Centre in Kings Cross: An Update, Sydney: NSW Bureau of Crime Statistics and Research, 2010. As of March 30, 2018: http://apo.org.au/node/22857

Fitzgerald, Therese C., Brendan Abel, and Sarah R. Bates, Establishment of a Pilot Medically Supervised Injection Facility In Massachusetts, Waltham: Massachusetts Medical Society, April 2017.

Fraser, Suzanne, “The Future of 'Addiction': Critique and Composition," International Journal of Drug Policy, Vol. 44, June 2017, pp. 130-134.

Freeman, K., C. G. Jones, D. J. Weatherburn, S. Rutter, C. J. Spooner, and N. Donnelly, "The Impact of the Sydney Medically Supervised Injecting Centre (MSIC) on Crime," Drug Alcohol Review, Vol. 24, No. 2, 2005, pp. 173-184.

Garcia, R. C., "Drug Consumption Rooms in Spain (2000-2013)," Salud Colectiva, Vol. 11, No. 2, 2015, pp. 261-282.

Gartry, C. C., E. Oviedo-Joekes, N. Laliberte, and M. T. Schechter, "NAOMI: The Trials and Tribulations of Implementing a Heroin Assisted Treatment Study in North America," Harm Reduction Journal, Vol. 6, No. 2, 2009.

Gonzalez v. Oregon, 546 U.S. 243, 2006.

Gonzalez v. Raich, 545 U.S. 1, 2005. 
Gowing, L., R. Ali, J. M. White, and D. Mbewe, "Buprenorphine for Managing Opioid Withdrawal," Cochrane Database of Systematic Reviews, No. 2, 2017.

Grey Literature Report, New York Academy of Medicine, "What Is Grey Literature?” webpage, undated. As of September 26, 2018:

http://www.greylit.org/about

Haasen, C., U. Verthein, P. Degkwitz, J. Berger, M. Krausz, and D. Naber, “Heroin-Assisted Treatment for Opioid Dependence: Randomised Controlled Trial," The British Journal of Psychiatry, Vol. 191, July 2007, pp. 55-62.

Hartnoll, R. L., M. C. Mitcheson, A. Battersby, G. Brown, M. Ellis, P. Fleming, and N. Hedley, "Evaluation of Heroin Maintenance in Controlled Trial," Archives of General Psychiatry, Vol. 37, No. 8, 1980, pp. 877-884.

Health Canada, "Supervised Consumption Sites: Status of Applications," webpage, updated February 21, 2018. As of February 21, 2018:

https://www.canada.ca/en/health-canada/services/substance-abuse/supervised-consumptionsites/status-application.html

Health Directorate, Norwegian Ministry of Health and Care Services, Prøveprosjekt:

Heroinassistert behandling-oppdrag nr. 34, Oslo, August 10, 2018. As of October 29, 2018:

https://www.regjeringen.no/contentassets/889319cb65664b63a9938b3273316033/2018/tilleg gsoppdrag_heroinassistert_behandling_180810.pdf

Heaton, P., Hidden in Plain Sight: What Cost-of-Crime Research Can Tell Us About Investing in Police, Santa Monica, Calif.: RAND Corporation, OP-279-ISEC, 2010. As of November 12, 2018:

https://www.rand.org/pubs/occasional_papers/OP279.html

Heckathorn, D. D. "Respondent-Driven Sampling: A New Approach to the Study of Hidden Populations," Social Problems, Vol. 44, No. 2, 1997, pp. 174-199.

Heckathorn, D. D., and C. J. Cameron, C. J., "Network Sampling: From Snowball and Multiplicity to Respondent-Driven Sampling," Annual Review of Sociology, Vol. 43, 2017, pp. 101-119.

Hedrich, Dagmar, European Report on Drug Consumption Rooms, Lisbon, Portugal: European Monitoring Centre for Drugs and Drug Addiction, February 2004.

Heins, S. E., M. J. Sorbero, C. M. Jones, A. W. Dick, and B. D. Stein, "High-Risk Prescribing to Medicaid Enrollees Receiving Opioid Analgesics: Individual-and County-Level Factors," Substance Use and Misuse, Vol. 53, No. 10, 2018, pp. 1591-1601. 
Hemming, K., T. P. Haines, P. J. Chilton, A. J. Girling, and R. J. Lilford, "The Stepped Wedge Cluster Randomised Trial: Rationale, Design, Analysis, and Reporting," BMJ, Vol. 350, 2015.

Hines v. Davidowitz, 312 U.S. 52, 1941.

Howard, D. L., N. J. Barrett, and D. N. Holmes, "Can Cultural Competency Speak to the Race Disparities in Methadone Dosage Levels?" The Review of Black Political Economy, Vol. 37, No. 1, 2010, pp. 7-23.

Hser, Yih-ing, Valerie Hoffman, Christine E. Grella, and M. Douglas Anglin, "A 33-Year Follow-Up of Narcotics Addicts," Archives of General Psychiatry, Vol. 58, No. 5, 2001, pp. 503-508.

International Journal of Drug Policy, "Retracted: The Impact of Medically Supervised Injection Centres on Drug-Related Harms: A Meta-Analysis," September 2018. As of November 2, 2018: https://www.ijdp.org/article/S0955-3959(18)30180-4/fulltext

Jehn, K. A., and L. Doucet, "Developing Categories from Interview Data: Text Analysis and Multidimensional Scaling, Part 1," Field Methods, Vol. 8, No. 2, 1996, pp. 15-16.

__ "Developing Categories for Interview Data: Consequences of Different Coding and Analysis Strategies in Understanding Text, Part 2," Field Methods, Vol. 9, No. 1, 1997, pp. $1-7$.

Joint Committee of the American Bar Association and the American Medical Association on Narcotic Drugs, Drug Addiction: Crime or Disease? Interim and Final Reports, Indianapolis: Indiana University Press, 1961.

Jones, Christopher M., Emily B. Einstein, and Wilson M. Compton, "Changes in Synthetic Opioid Involvement in Drug Overdose Deaths in the United States, 2010-2016," JAMA, Vol. 319, No. 17, 2018, pp. 1819-1821.

Jones, E. S., B. A. Moore, J. L. Sindelar, P. G. O'Connor, R. S. Schottenfeld, and D. A. Fiellin, "Cost Analysis of Clinic and Office-Based Treatment of Opioid Dependence: Results with Methadone and Buprenorphine in Clinically Stable Patients," Drug and Alcohol Dependence, Vol. 99, No. 1-3, 2009, pp. 132-140.

Jozaghi, Ehsan, "A Cost-Benefit/Cost-Effectiveness Analysis of an Unsanctioned Supervised Smoking Facility in the Downtown Eastside of Vancouver, Canada," Harm Reduction Journal, Vol. 11, No. 1, 2014.

Judson, Horace, Heroin Addiction: What Americans Can Learn from the English Experience, New York: Harcourt Brace Jovanovich, 1974. 
Kappel, N., E. Toth, J. Tegner, and S. Lauridsen, "A Qualitative Study of How Danish Drug Consumption Rooms Influence Health and Well-Being Among People Who Use Drugs," Harm Reduction Journal, Vol. 13, No. 20, 2016.

Kennedy, M. C., M. Karamouzian, and T. Kerr, "Public Health and Public Order Outcomes Associated with Supervised Drug Consumption Facilities: A Systematic Review," Current HIV/AIDS Reports, Vol. 14, No. 5, 2017, pp. 161-183.

Kerr, T., J. Kimber, K. Debeck, and E. Wood, "The Role of Safer Injection Facilities in the Response to HIV/AIDS Among Injection Drug Users," Current HIV/AIDS Reports, Vol. 4, No. 4, 2007, pp. 158-164.

Kerr, T., W. Small, D. Moore, and E. Wood, "A Micro-Environmental Intervention to Reduce the Harms Associated with Drug-Related Overdose: Evidence from the Evaluation of Vancouver's Safer Injection Facility," International Journal on Drug Policy, Vol. 18, 2007, pp. 37-45.

Kerr, Thomas, Mark Tyndall, Kathy Li, Julio Montaner, and Evan Wood, "Safer Injection Facility Use and Syringe Sharing in Injection Drug Users," The Lancet, Vol. 366, No. 9482, 2005, pp. 316-318.

Khan, R., Y. Khazaal, G. Thorens, D. Zullino, and A. Uchtenhagen, "Understanding Swiss Drug Policy Change and the Introduction of Heroin Maintenance Treatment," European Addiction Research, Vol. 20, No. 4, 2014, pp. 200-207.

Kimber, J., K. Dolan, I. Van Beek, D. Hedrich, and H. Zurhold, "Drug Consumption Facilities: An Update Since 2000," Drug and Alcohol Review, Vol. 22, No. 2, 2003, pp. 227-233.

Kilmer, Beau, Susan S. Sohler Everingham, Jonathan P. Caulkins, Greg Midgette, Rosalie Liccardo Pacula, Peter H. Reuter, Rachel M. Burns, Bing Han, and Russell Lundberg, What America's Users Spend on Illegal Drugs: 2000-2010, Santa Monica, Calif.: RAND Corporation, RR-534-ONDCP, 2014. As of November 12, 2018:

https://www.rand.org/pubs/research_reports/RR534.html

Kolla, G., C. Strike, T. M. Watson, J. Jairam, B. Fischer, and A. M. Bayoumi, "Risk Creating and Risk Reducing: Community Perceptions of Supervised Consumption Facilities for Illicit Drug Use," Health, Risk and Society, Vol. 19, Nos. 1-2, 2017, pp. 91-111.

Kolodny, Andrew, David T. Courtwright, Catherine S. Hwang, Peter Kreiner, John L. Eadie, Thomas W. Clark, and G. Caleb Alexander, "The Prescription Opioid and Heroin Crisis: A Public Health Approach to an Epidemic of Addiction," Annual Review of Public Health, Vol. 36, 2015, pp. 559-574.

Kral, Alex H., and Peter J. Davidson, “Addressing the Nation's Opioid Epidemic: Lessons from an Unsanctioned Supervised Injection Site in the U.S.," American Journal of Preventive Medicine, Vol. 53, No. 6, 2017, pp. 919-922. 
Kral, A. H., L. Wenger, L. Carpenter, E. Wood, T. Kerr, and P. Bourgois, "Acceptability of a Safer Injection Facility Among Injection Drug Users in San Francisco," Drug and Alcohol Dependence, Vol. 110, Nos. 1-2, 2010, pp. 160-163.

Kreit, Alex, "Safe Injection Sites and the Federal 'Crack House' Statute," Boston College Law Review, forthcoming.

Krusi, A., W. Small, E. Wood, and T. Kerr, "An Integrated Supervised Injecting Program Within a Care Facility for HIV-Positive Individuals: A Qualitative Evaluation," AIDS Care, Vol. 21, No. 5, 2009, pp. 638-644.

Lasnier, B., S. Brochu, N. Boyd, and B. Fischer, "A Heroin Prescription Trial: Case Studies from Montreal and Vancouver on Crime and Disorder in the Surrounding Neighbourhoods," International Journal on Drug Policy, Vol. 21 No. 1, 2010, pp. 28-35.

L.B. v. Town of Chester, 232 F. Supp. 2d 227, Southern District of New York, 2002

Lobmaier, P., H. Kornor, N. Kunoe, and A. Bjørndal, "Sustained-Release Naltrexone for Opioid Dependence," Cochrane Database of Systematic Reviews, No. 2, April 2008.

Lopez, German, "Why Some U.S. Cities Are Opening Safe Spaces for Injecting Heroin," Vox, September 17, 2018. As of November 2, 2018 :

https://www.vox.com/science-and-health/2018/1/25/16928144/safe-injection-sites-heroinopioid-epidemic

Lupick, Travis, "To Curb Fatal Drug Overdoses, the U.S. Urgently Needs Safe Injection Sites: The Problem Is They're Illegal,” Los Angeles Times, July 27, 2018. As of November 2, 2018: http://www.latimes.com/opinion/op-ed/la-oe-lupick-opioid-epidemic-supervised-injectionsites-20180727-story.html

MacCoun, Robert J., and Peter Reuter, Drug War Heresies: Learning from Other Vices, Times, and Places, Cambridge, UK: Cambridge University Press, 2001.

__ "Assessing Drug Prohibition and Its Alternatives: A Guide for Agnostics," Annual Review of Law and Social Science, Vol. 7, 2011, pp. 61-78.

Magill, Molly, and Lara A. Ray, "Cognitive-Behavioral Treatment with Adult Alcohol and Illicit Drug Users: A Meta-Analysis of Randomized Controlled Trials," Journal of Studies on Alcohol and Drugs, Vol. 70, No. 4, 2009, pp. 516-527.

March, J. C., E. Oviedo-Joekes, E. Perea-Milla, and F. Carrasco, "Controlled Trial of Prescribed Heroin in the Treatment of Opioid Addiction," Journal of Substance Abuse Treatment, Vol. 31, No. 2, 2006, pp. 203-211.

Mars, S. G., P. Bourgois, G. Karandinos, F. Montero, and D. Ciccarone, "Every 'Never' I Ever Said Came True: Transitions from Opioid Pills to Heroin Injecting," International Journal of Drug Policy, Vol. 25, No. 2, 2014, pp. 257-266. 
Mars, Sarah G., Jeff Ondocsin, and Daniel Ciccarone, "Sold as Heroin: Perceptions and Use of an Evolving Drug in Baltimore, MD,” Journal of Psychoactive Drugs, Vol. 50, No. 2, 2018, pp. 167-176.

Marshall, Brendan, M. J. Milloy, E. Wood, J. S. Montaner, and T. Kerr, "Reduction in Overdose Mortality After the Opening of North America's First Medically Supervised Safer Injecting Facility: A Retrospective Population-Based Study," Lancet, Vol. 377, No. 9775, 2011, pp. $1429-1437$.

Masson, C. L., P. G. Barnett, K. L. Sees, K. L. Delucchi, A. Rosen, W. Wong, and S. M. Hall, "Cost and Cost-Effectiveness of Standard Methadone Maintenance Treatment Compared to Enriched 180-Day Methadone Detoxification,” Addiction, Vol. 99, No. 6, 2004, pp. 718-726.

Mattick, R. P., C. Breen, J. Kimber, and M. Davoli, "Methadone Maintenance Therapy Versus No Opioid Replacement Therapy for Opioid Dependence," Cochrane Database of Systematic Reviews, No. 3, July 2009.

May, T., T. Bennett, and K. Holloway, K. "The Impact of Medically Supervised Injection Centres on Drug-Related Harms: A Meta-Analysis," International Journal of Drug Policy, Vol. 59, 2018, pp. 98-107.

McGinty, E. E., C. L. Barry, E. M. Stone, J. Niederdeppe, A. Kennedy-Hendricks, S. Linden, and S. G. Sherman, "Public Support for Safe Consumption Sites and Syringe Services Programs to Combat the Opioid Epidemic," Preventive Medicine, Vol. 111, 2018, pp. 73-77.

McNeil, R., and W. Small, “'Safer Environment Interventions': A Qualitative Synthesis of the Experiences and Perceptions of People Who Inject Drugs," Social Science and Medicine, Vol. 106, 2014, pp. 151-158.

Metrebian, Nicky, Tom Carnwath, Gerry V. Stimson, and Thomas Storz, "Survey of Doctors Prescribing Diamorphine (Heroin) to Opiate-Dependent Drug Users in the United Kingdom," Addiction, Vol. 97, No. 9, 2002, pp. 1155-1161.

Mikos, Robert A., "Preemption Under the Controlled Substances Act," Journal of Health Care Law and Policy, Vol. 16, No. 1, 2013.

Miles, Matthew B., and A. Michael Huberman, Qualitative Data Analysis: An Expanded Sourcebook, 2nd ed., Thousand Oaks, Calif.: SAGE Publications, 1994.

Miller, P., S. McKenzie, N. Lintzeris, A. Martin, and J. Strang, "The Community Impact of RIOTT, a Medically Supervised Injectable Maintenance Clinic in south London," Mental Health and Substance Use: Dual Diagnosis, Vol. 3, No. 3, 2010, pp. 248-259.

Miller, P., S. McKenzie, J. Walker, N. Lintzeris, and J. Strang, "Investigating the Effect on Public Behaviour of Patients of a Medically Supervised Injectable Maintenance Clinic," Drugs and Alcohol Today, Vol. 11, No. 4, 2011, pp. 204-209. 
Milloy, M-J. S., Thomas Kerr, Mark Tyndall, Julio Montaner, and Evan Wood, "Estimated Drug Overdose Deaths Averted by North America's First Medically-Supervised Safer Injection Facility," PLoS One, Vol. 3, No. 10, 2008.

Mitra, S., B. Rachlis, A. Scheim, G. Bardwell, S. B. Rourke, and T. Kerr, “Acceptability and Design Preferences of Supervised Injection Services Among People Who Inject Drugs in a Mid-Sized Canadian City," Harm Reduction Journal, Vol. 14, No. 1, 2017.

Musto, David, The American Disease, 3rd. ed., New York: Oxford University Press, 1999.

Myer, A. J., and L. Belisle, "Highs and Lows: An Interrupted Time-Series Evaluation of the Impact of North America's Only Supervised Injection Facility on Crime," Journal of Drug Issues, Vol. 48, No. 1, 2018, pp. 36-49.

National Academies of Sciences, Engineering, and Medicine, Pain Management and the Opioid Epidemic: Balancing Societal and Individual Benefits and Risks of Prescription Opioid Use, Washington, D.C.: The National Academies Press, 2017.

National Bioethics Advisory Commission, Research Involving Subjects with Mental Disorders That May Affect Decisionmaking Capacity, Rockville, Md., 1998.

National Centre in HIV Epidemiology and Clinical Research, Sydney Medically Supervised Injecting Centre Evaluation Report No. 4: Evaluation of Service Operation and OverdoseRelated Events, Sydney, 2007. As of November 2, 2018:

https://kirby.unsw.edu.au/sites/default/files/kirby/report/EvalRep4SMSIC.pdf

National Institute on Drug Abuse, Principles of Drug Addiction Treatment: A Research-Based Guide, 3rd ed., Bethesda, Md., January 2018a.

— , “How Much Does Opioid Treatment Cost?” webpage, last updated June 2018b. As of November 2, 2018:

https://www.drugabuse.gov/publications/research-reports/medications-to-treat-opioidaddiction/how-much-does-opioid-treatment-cost

New York v. United States, 505 U.S. 144, 1992.

New York State, McKinney's Consolidated Laws of New York Annotated: With Annotations from State and Federal Courts and State Agencies, St. Paul, Minn: West Group, 2008.

New York State Senate, Consolidated Laws of New York, webpage, undated. As of November 12, 2018:

https://www.nysenate.gov/legislation/laws/CONSOLIDATED

Nordlund, D. J., S. Estee, D. Mancuso, and B. Felver, "Methadone Treatment for Opiate Addiction Lowers Health Care Costs and Reduces Arrests and Convictions," fact sheet, Olympia, Wash.: Washington State Dept. of Social and Health Services, Research and Data Analysis Division, 2004. 
Nosyk, Bohdan, Daphne P. Guh, Nicholas J. Bansback, Eugenia Oviedo-Joekes, Suzanne Brissette, David C. Marsh, Evan Meikleham, Martin T. Schechter, and Aslam H. Anis, "Cost-Effectiveness of Diacetylmorphine Versus Methadone for Chronic Opioid Dependence Refractory to Treatment," CMAJ, 2012.

Ober, A. J., J. Sussell, B. Kilmer, J. Saunders, and D. D. Heckathorn, "Using Respondent-Driven Sampling to Recruit Illegal Drug Purchasers to Evaluate a Drug Market Intervention," Evaluation Review, Vol. 40, No. 2, 2016, pp. 87-121.

Ober, Allison J., Jirka Taylor, Martin Iguchi, and Jonathan P. Caulkins, Acceptability of HeroinAssisted Treatment and Supervised Drug Consumption Sites to Address the Opioid Crisis in the United States: Key Informant Perspectives, Santa Monica, Calif.: RAND Corporation, WR-1260-RC, 2018. As of December 6, 2018: http://www.rand.org/pubs/working_papers/WR1260

Ogden, David W., "Investigations and Prosecutions in States Authorizing the Medical Use of Marijuana," memorandum for select U.S. attorneys, October 19, 2009.

Oviedo-Joekes, E., S. Brissette, D. C. Marsh, P. Lauzon, D. Guh, A. Anis, and M. T. Schechter, "Diacetylmorphine Versus Methadone for the Treatment of Opioid Addiction," New England Journal of Medicine, Vol. 361, No. 8, 2009, pp. 777-786.

Oviedo-Joekes, E., D. Guh, S. Brissette, K. Marchand, S. MacDonald, K. Lock, S. Harrison, A. Janmohamed, A. H. Anis, M. Krausz, D. C. Marsh, and M. T. Schechter, "Hydromorphone Compared with Diacetylmorphine for Long-Term Opioid Dependence: A Randomized Clinical Trial," JAMA Psychiatry, Vol. 73, No. 5, 2016, pp. 447-455.

Owoseje, Toyin, "Norway to Give Hundreds of Drug Addicts Free Heroin to 'Improve Quality of Life,"' Independent, August 10, 2018. As of November 2, 2018:

https://www.independent.co.uk/life-style/health-and-families/health-news/norway-freeheroin-drug-addicts-treatment-care-a8486571.html

Pacula, R. L., and D. Powell, “A Supply-Side Perspective on the Opioid Crisis," Journal of Policy Analysis and Management, Vol. 37, No. 2, 2018a, pp. 438-446.

—, "Responses to Questions on Policing and Insurance Benefit Design as They Pertain to the Opioid Crisis," Journal of Policy Analysis and Management, Vol. 37, No. 2, 2018b, pp. $449-451$.

Palmateer, Norah, Jo Kimber, Matthew Hickman, Sharon Hutchinson, Tim Rhodes, and David Goldberg, "Evidence for the Effectiveness of Sterile Injecting Equipment Provision in Preventing Hepatitis $\mathrm{C}$ and Human Immunodeficiency Virus Transmission Among Injecting Drug Users: A Review of Reviews," Addiction, Vol. 105, No. 5, 2010, pp. 844-859.

Paquette, D., J. Bryant, and J. De Wit, "Respondent-Driven Sampling and the Recruitment of People with Small Injecting Networks," AIDS and Behavior, Vol. 16, No. 4, 2012, pp. 890899. 
Pardo, Bryce, Jonathan P. Caulkins, and Beau Kilmer, Assessing the Evidence on Supervised Drug Consumption Sites, Santa Monica, Calif.: RAND Corporation, WR-1261-RC, 2018. As of December 6, 2018:

http://www.rand.org/pubs/working_papers/WR1261

Pardo, B., and P. Reuter, P., "Facing Fentanyl: Should the USA Consider Trialling Prescription Heroin?" Lancet: Psychiatry, Vol. 5, No. 8, 2018, pp. 613-615.

Pearson, Helen, "Science and the War on Drugs: A Hard Habit to Break," Nature, Vol. 430, No. 6998, July 22, 2004, p. 394-395.

Peng, P. W., and A. N. Sandler, "A Review of the Use of Fentanyl Analgesia in the Management of Acute Pain in Adults," Journal of the American Society of Anesthesiologists, Vol. 90, No. 2, 1999, pp. 576-599.

People v. Crouse, 412 P.3d 599, Court of Appeals of Colorado, 2013.

Perneger, T. V., F. Giner, M. del Rio, and A. Mino, "Randomised Trial of Heroin Maintenance Programme for Addicts Who Fail in Conventional Drug Treatments," BMJ, Vol. 317, No. 7150, 1998, pp. 13-18.

Pinkerton, Steven D., "Is Vancouver Canada's Supervised Injection Facility Cost-Saving?" Addiction, Vol. 105, No. 8, 2010, pp. 1429-1436.

__ "How Many HIV Infections Are Prevented by Vancouver Canada's Supervised Injection Facility?" International Journal of Drug Policy, Vol. 22, No. 3, 2011, pp. 179-183.

Pollini, R. A., C. J. Banta-Green, J. Cuevas-Mota, M. Metzner, E. Teshale, and R. S. Garfein, "Problematic Use of Prescription-Type Opioids Prior to Heroin Use Among Young Heroin Injectors," Substance Abuse and Rehabilitation, Vol. 2, No. 1, October 2011, pp. 173-180.

Potier, C., V. Laprevote, F. Dubois-Arber, O. Cottencin, and B. Rolland, "Supervised Injection Services: What Has Been Demonstrated? A Systematic Literature Review," Drug and Alcohol Dependence, Vol. 145, 2014, pp. 48-68.

Printz v. United States, 521 U.S. 898, 1997.

Providence Health Care Society v. Canada, 2014 BCSC 936, British Columbia, Canada, 2014.

Public Law 115-141, Consolidated Appropriations Act, 2018, March 23, 2018.

Quinnipiac University, "U.S. Voters Believe Comey More Than Trump, Quinnipiac University National Poll Finds; Support for Marijuana Hits New High,” April 26, 2018. As of November 2, 2018:

https://poll.qu.edu/national/release-detail?ReleaseID=2539

Rangel, Charles B., "Heroin Maintenance-Solving the Drug Crisis or Serving It?" Congressional Record, Vol. 118, No. 4, February 17, 1972, p. 4682. 
"Residential Opioid Program Giving Drug Users Chance at New Life," Eastern Ontario Network Television, June 27, 2018. As of November 26, 2018:

http://easternontarionetwork.com/2018/06/27/residential-opioid-program-giving-drug-userschance-at-new-life/

Reuter, P., and R. MacCoun, "Heroin Maintenance: Is a U.S. Experiment Needed?" in D. Musto, ed., One Hundred Years of Heroin, Westport, Conn.: Greenwood, 2002, pp. 159-180.

Rolleston Report, 1926 Report of the Departmental Committee on Morphine and Heroin Addiction, London: Her Majesty's Stationery Office, 1926.

Romero, Carolina, "Mexicali Offers Safe Spot for Heroin Addicts," El Universal, November 23, 2018. As of November 26, 2018:

http://www.eluniversal.com.mx/english/mexicali-offers-safe-spot-for-heroin-addicts

Rosenheck, R., and T. Kosten, "Buprenorphine for Opiate Addiction: Potential Economic Impact," Drug and Alcohol Dependence, Vol. 63, No. 3, 2001, pp. 253-262.

Rosenstein, Rod J., "Fight Drug Abuse, Don't Subsidize It," New York Times, August 27, 2018. As of November 5, 2018:

https://www.nytimes.com/2018/08/27/opinion/opioids-heroin-injection-sites.html

Rowe, C., G. M., Santos, E. Vittinghoff, E. Wheeler, P. Davidson, and P. O. Coffin, "Neighborhood-Level and Spatial Characteristics Associated with Lay Naloxone Reversal Events and Opioid Overdose Deaths," Journal of Urban Health, Vol. 93, No. 1, 2016, pp. $117-130$.

Rowe, C., E. Wheeler, T. Stephen Jones, C. Yeh, and P. O. Coffin, "Community-Based Response to Fentanyl Overdose Outbreak, San Francisco, 2015," Journal of Urban Health, 2018.

Ruhm, Christopher J., "Corrected U.S. Opioid-Involved Drug Poisoning Deaths and Mortality Rates, 1999-2015," Addiction, Vol. 113, No. 7, 2018.

Ryan, Gery W., and H. Russell Bernard, "Data Management and Analysis Methods," in Norman Denzin and Yvonna Lincoln, eds., Handbook of Qualitative Research, 2nd ed., Thousand Oaks, Calif.: SAGE Publications, 2000, pp. 769-802.

__, "Techniques to Identify Themes," Field Methods, Vol. 15, No. 1, 2003, pp. 85-110.

Salmon, A. M., I. van Beek, J. Amin, J. Kaldor, and L. Maher, “The Impact of a Supervised Injecting Facility on Ambulance Call-Outs in Sydney, Australia," Addiction, Vol. 105, No. 4, 2010, pp. 676-683.

Saloner, B., and C. L. Barry, "Response to Pacula and Powell: Investing in Harm Reduction and Alternatives to Coerced Treatment," Journal of Policy Analysis and Management, Vol. 37, No. 2, 2018, pp. 446-449. 
San Diego Countyv. San Diego NORML, 81 Cal. Rptr. 461, 129 S.Ct. 2380, 2009.

Sherman, Susan, Kyle Hunter, and Saba Rouhani, Safe Drug Consumption Spaces: A Strategy for Baltimore City, Baltimore, Md.: Abell Foundation, February 2017. As of November 4, 2018:

https://www.abell.org/sites/default/files/files/Safe\%20Drug\%20Consumption\%20Spaces\%20 final.pdf

Small, W., N. Van Borek, N. Fairbairn, E. Wood, and T. Kerr, "Access to Health and Social Services for IDU: The Impact of a Medically Supervised Injection Facility,” Drug and Alcohol Review, Vol. 28, No. 4, 2009, pp. 341-346.

Smart, Rosanna, Evidence on the Effectiveness of Heroin-Assisted Treatment, Santa Monica, Calif.: RAND Corporation, WR-1263-RC, 2018. As of December 6, 2018: http://www.rand.org/pubs/working_papers/WR1263

Smith v. Hickenlooper, 164 F. Supp. 3d 1286, District Court of Colorado, 2016.

Spokane County Health District v. Brockett, 839 P.2d 324, Washington, 1992.

Strang, J., N. Metrebian, N. Lintzeris, L. Potts, T. Carnwath, S. Mayet, H. Williams, D. Zador, R. Evers, T. Groshkova, V. Charles, A. Martin, and L. Forzisi, "Supervised Injectable Heroin or Injectable Methadone Versus Optimised Oral Methadone as Treatment for Chronic Heroin Addicts in England After Persistent Failure in Orthodox Treatment (RIOTT): A Randomised Trial," Lancet, Vol. 375, No. 9729, 2010, pp. 1885-1895.

Strang, Lucy, and Jirka Taylor, Heroin-Assisted Treatment and Supervised Drug Consumption Sites: Experience from Four Countries, Santa Monica, Calif.: RAND Corporation, WR1262-RC, 2018. As of December 6, 2018:

http://www.rand.org/pubs/working_papers/WR1262

Strike, Carol, Jennifer A. Jairam, Gillian Kolla, Peggy Millson, Susan Shepherd, Benedikt Fischer, Tara Marie Watson, and Ahmed M. Bayoumi, "Increasing Public Support for Supervised Injection Facilities in Ontario, Canada," Addiction, Vol. 109, No. 6, 2014, pp. 946-953.

Strike, Carol, Tara Marie Watson, Gillian Kolla, Rebecca Penn, and Ahmed M. Bayoumi, "Ambivalence About Supervised Injection Facilities Among Community Stakeholders," Harm Reduction Journal, Vol. 12, No. 1, 2015.

Substance Abuse and Mental Health Services Administration, Key Substance Use and Mental Health Indicators in the United States: Results from the 2016 National Survey on Drug Use and Health, Rockville, Md., 2017. As of November 4, 2018: https:/www.samhsa.gov/data/sites/default/files/NSDUH-FFR1-2016/NSDUH-FFR12016.htm 
Tanum, L., K. K. Solli, J. S. Benth, A. Opheim, K. Sharma-Haase, P. Krajci, and N. Kunøe, "Effectiveness of Injectable Extended-Release Naltrexone vs. Daily BuprenorphineNaloxone for Opioid Dependence: A Randomized Clinical Noninferiority Trial," JAMA Psychiatry, Vol. 74, No. 12, 2017, pp. 1197-1205.

Tempalski, B., "Placing the Dynamics of Syringe Exchange Programs in the United States," Health and Place, Vol. 13, No. 2, 2007, pp. 417-431.

Ter Beek v. City of Wyoming, 846 N.W.2d 531, Michigan, 2014.

Town of Wakefield v. Coakley, 2014 Mass. Super. LEXIS 2023, Massachusetts, 2014.

Uchtenhagen, Ambros, "The Role and Function of Heroin Assisted Treatment at the Treatment System Level," Heroin Addiction and Related Clinical Problems, Vol. 19, No. 2, 2017.

Uchtenhagen, A., A. Dobler-Mikola, T. Steffen, F. Gutzwiller, R. Blattler, and S. Pfeiffer, "Prescription of Narcotics for Heroin Addicts: Main Results of the Swiss National Cohort Study," in Medical Prescription of Narcotics, Vol. 1, Basel, Switzerland: Karger, 1999.

UK Home Office Drugs Strategy Directorate, Updated Drug Strategy 2002, London: Crown, 2002.

United Nations, Single Convention on Narcotic Drugs, New York, March 30, 1961.

United States v. Chen, 913 F.2d 183, U.S. Court of Appeals, 5th Cir., 1990.

United States Code, Title 18, Section 2, Principals.

— Title 21, Section 801, Congressional Findings and Declarations: Controlled Substances.

—

—, Title 21, Section 823, Registration Requirements.

— Title 21, Section 844, Penalties for Simple Possession.

—- Title 21, Section 846, Attempt and Conspiracy.

—, Title 21, Section 856, Maintaining Drug-Involved Premises.

—, Title 21, Section 871, Attorney General.

—, Title 21, Section 881, Forfeitures.

— Title 21, Section 903, Application of State Law.

—

UK Parliament, Dangerous Drugs Act of 1967, London, 1967.

U.S. Census Bureau, "ACS Demographic and Household Estimates," 2012-2016 American Community Survey 5-Year Estimates, 2018.

U.S. Constitution, Art. VI, Cl. 2. 
U.S. Department of Justice, U.S. Attorney's Office, District of Vermont, "Statement of the U.S. Attorney's Office Concerning Proposed Injection Sites," December 13, 2017. As of November 4, 2018:

https://www.justice.gov/usao-vt/pr/statement-us-attorney-s-office-concerning-proposedinjection-sites

U.S. Food and Drug Administration, Consulting the Controlled Substance Staff on INDs and Protocols That Use Schedule I Controlled Substances and Drugs, Manual of Policies and Procedures 4200.1, Washington, D.C., May 8, 2003. As of November 12, 2018 : https://www.fda.gov/downloads/AboutFDA/CentersOffices/OfficeofMedicalProductsandTob acco/CDER/ManualofPoliciesProcedures/UCM073577.pdf

Vancouver Coastal Health, "Insite User Statistics," September 2018. As of November 4, 2018: http://www.vch.ca/public-health/harm-reduction/supervised-consumption-sites/insite-userstatistics

van den Brink, W., and P. Blanken, Medical Co-Prescription of Heroin: Two Randomized Controlled Trials, Utrecht, Netherlands: Central Committee on the Treatment of Heroin Addicts, 2002.

van den Brink, W., V. M. Hendriks, P. Blanken, M. W. Koeter, B. J. van Zwieten, and J. M. van Ree, "Medical Prescription of Heroin to Treatment Resistant Heroin Addicts: Two Randomised Controlled Trials," BMJ, Vol. 327, No. 7410, 2003.

Vancouver Coastal Health, "Insite User Statistics," webpage, February 2018. As of November 12, 2018:

http://www.vch.ca/public-health/harm-reduction/supervised-consumption-sites/insite-userstatistics

Vecino, C., J. R. Villalbi, A. Guitart, A. Espelt, M. Bartroli, Y. Castellano, and M. T. Brugal, "Safe Injection Rooms and Police Crackdowns in Areas with Heavy Drug Dealing: Evaluation by Counting Discarded Syringes Collected from the Public Space," Adicciones, Vol. 25, No. 4, 2013, pp. 333-338.

Washburn v. Columbia Forest Prods., Inc., 134 P.3d 161, Oregon, 2006.

Washington State Institute for Public Policy, "Methadone Maintenance for Opioid Use Disorder," webpage, December 2017. As of November 26, 2018 :

http://www.wsipp.wa.gov/BenefitCost/Program/694

Watson, T. M., A. Bayoumi, G. Kolla, R. Penn, B. Fischer, J. Luce, and C. Strike, "Police Perceptions of Supervised Consumption Sites (SCSs): A Qualitative Study," Substance Use and Misuse, Vol. 47, No. 4, 2012, pp. 364-374.

Watson, T. M., C. Strike, G. Kolla, R. Penn, and A. M. Bayoumi, “Drugs Don't Have Age Limits: The Challenge of Setting Age Restrictions for Supervised Injection Facilities," Drugs: Education, Prevention and Policy, Vol. 22, No. 4, 2015, pp. 370-379. 
Watson, T. M., C. Strike, G. Kolla, R. Penn, J. Jairam, S. Hopkins, and A. M. Bayoumi, "Design Considerations for Supervised Consumption Facilities (SCFs): Preferences for Facilities Where People Can Inject and Smoke Drugs," International Journal of Drug Policy, Vol. 24, No. 2, 2013, pp. 156-163.

Weatherburn, Don, Craig Jones, Karen Freeman, and Toni Makkai, "Supply Control and Harm Reduction: Lessons from the Australian Heroin 'Drought,"' Addiction, Vol. 98, No. 1, 2002, pp. 83-91.

Weeks, Carly, "Opioid Overdose Deaths Push Down Life Expectancy in B.C.," Globe and Mail, October 23, 2018. As of November 4, 2018:

https://www.theglobeandmail.com/canada/article-opioid-overdoses-lead-to-fall-in-lifeexpectancy-in-bc-threatens/

Wenger, L. D., S. G. Arreola, and A. H. Kral, "The Prospect of Implementing a Safer Injection Facility in San Francisco: Perspectives of Community Stakeholders," International Journal of Drug Policy, Vol. 22, No. 3, 2011, pp. 239-241.

Wheeler, E., T. S. Jones, M. K. Gilbert, and P. J. Davidson, “Opioid Overdose Prevention Programs Providing Naloxone to Laypersons - United States, 2014," Morbidity and Mortality Weekly Report, Vol. 64, No. 23, 2015, pp. 631-635.

Wherry, Aaron, "Debate over Drug Consumption Sites Might Be Coming to End," CBC News, November 5, 2017. As of February 21, 2018:

http://www.cbc.ca/news/politics/consumption-sites-heroin-opioid-analysis-wherry1.4280612

Willms, Dennis G., J. Allan Best, D. Wayne Taylor, J. Raymond Gilbert, Douglas M. C. Wilson, Elizabeth A. Lindsay, and Joel Singer, "A Systematic Approach for Using Qualitative Methods in Primary Prevention Research," Medical Anthropology Quarterly, Vol. 4, No. 4, 1990, pp. 391-409.

Wilson, Tiffany M., "LEAD the Way: Using Local Communities as Legal Laboratories to Combat Drug Addiction," Journal of Legal Medicine, Vol. 38, No. 1, 2018, pp. 163-176.

Wuethrich, Sandra, and Sektion Drogen, Handbuch diacetylmorphingestützte Behandlung: Richtlinien und Erläuterungen zu den gesetzlichen Bestimmungen, Koniz, Switzerland: Bundesamt für Gesundheit, April 2015. As of November 4, 2018:

https://www.bag.admin.ch/dam/bag/de/dokumente/npp/drogen/sucht/hegebe/handbuchhegebe.pdf.download.pdf/151020_Handbuch_D.pdf 